\title{
ON THE VARIETY OF LAGRANGIAN SUBALGEBRAS, I
}

\author{
BY SAM EVENS ${ }^{1}$ AND JIANG-HUA LU ${ }^{2}$
}

\begin{abstract}
We study subalgebras of a semi-simple Lie algebra which are Lagrangian with respect to the imaginary part of the Killing form. We show that the variety $\mathcal{L}$ of Lagrangian subalgebras carries a natural Poisson structure $\Pi$. We determine the irreducible components of $\mathcal{L}$, and we show that each irreducible component is a smooth fiber bundle over a generalized flag variety, and that the fiber is the product of the set of real points of a De Concini-Procesi compactification and a connected component of a real orthogonal group. We study some properties of the Poisson structure $\Pi$ and show that $\mathcal{L}$ contains many interesting Poisson submanifolds.
\end{abstract}

@ 2001 Éditions scientifiques et médicales Elsevier SAS

RÉSUMÉ. - Nous étudions les sous-algèbres d'une algèbre de Lie semi-simple qui sont lagrangiennes par rapport à la partie imaginaire de la forme de Killing. Nous montrons que la variété, $\mathcal{L}$, de ces sous-algèbres possède une structure de Poisson naturelle, $\Pi$. Nous décrivons les composantes irréductibles de $\mathcal{L}$ et nous montrons que chaque composante irréductible est un fibré lisse de base une variété de drapeaux généralisée et de fibre le produit des points réels d'une compactification de De Concini-Procesi par une composante connexe d'un groupe orthogonal. Nous étudions la structure de Poisson $\Pi$, et nous montrons que $\mathcal{L}$ contient des sous-variétés de Poisson intéressantes.

๑ 2001 Éditions scientifiques et médicales Elsevier SAS

\section{Introduction}

Let $\mathfrak{g}$ be a complex semi-simple Lie algebra and let $\operatorname{Im}\langle\langle\rangle$,$\rangle be the imaginary part of the$ Killing form $\langle\langle\rangle$,$\rangle of \mathfrak{g}$. We will say that a real subalgebra $\mathfrak{l}$ of $\mathfrak{g}$ is Lagrangian if $\operatorname{dim}_{\mathbb{R}} \mathfrak{l}=\operatorname{dim}_{\mathbb{C}} \mathfrak{g}$ and if $\operatorname{Im}\langle\langle x, y\rangle\rangle=0$ for all $x, y \in \mathfrak{l}$.

In this paper, we study the geometry of the variety $\mathcal{L}$ of Lagrangian subalgebras of $\mathfrak{g}$ and show that $\mathcal{L}$ carries a natural Poisson structure $\Pi$. We show that each irreducible component of $\mathcal{L}$ is smooth and is a fiber bundle over a generalized flag variety, and the fiber is the product of the set of real points of a De Concini-Procesi compactification and a connected component of a real orthogonal group. We study some properties of the Poisson structure $\Pi$ and show that it contains many interesting Poisson submanifolds.

The Poisson structure $\Pi$ is defined using the fact that $\mathfrak{g}$, regarded as a real Lie algebra, is the double of a Lie bialgebra structure on a compact real form $\mathfrak{k}$ of $\mathfrak{g}$. The construction of $\Pi$ works for any Lie bialgebra, and we present it in the first part of the paper. In the second part, we study the specific example of $\mathcal{L}$, which we regard as the most important example since it is closely related to interesting problems in Lie theory.

\footnotetext{
${ }^{1}$ Research partially supported by NSF grants DMS-9623322 and DMS-9970102.

${ }^{2}$ Research partially supported by NSF grant DMS 9803624. 
We now explain our motivation and give more details of our results.

Let $\left(\mathfrak{u}, \mathfrak{u}^{*}\right)$ be any Lie bialgebra, let $\mathfrak{d}$ be its double, and let $\langle$,$\rangle be the symmetric scalar$ product on $\mathfrak{d}$ given by:

$$
\langle x+\xi, y+\eta\rangle=(x, \eta)+(y, \xi), \quad x, y \in \mathfrak{u}, \xi, \eta \in \mathfrak{u}^{*} .
$$

A subalgebra $\mathfrak{l}$ of $\mathfrak{d}$ is said to be Lagrangian if $\operatorname{dim} \mathfrak{l}=\operatorname{dim} \mathfrak{u}$ and if $\langle a, b\rangle=0$ for all $a, b \in \mathfrak{l}$. Denote by $\mathcal{L}(\mathfrak{d})$ the set of all Lagrangian subalgebras of $\mathfrak{d}$. It is a subvariety of the Grassmannian of $n$-dimensional subspaces of $\mathfrak{d}$, where $n=\operatorname{dim} \mathfrak{u}$. The motivation for studying $\mathcal{L}(\mathfrak{d})$ comes from a theorem of Drinfeld [6] on Poisson homogeneous spaces which we now recall briefly. More details are given in Section 2.1.

Let $\left(U, \pi_{U}\right)$ be a Poisson Lie group with $\left(\mathfrak{u}, \mathfrak{u}^{*}\right)$ as its tangent Lie bialgebra. Recall that an action of $U$ on a Poisson manifold $(M, \pi)$ is called Poisson if the action map $U \times M \rightarrow M$ is a Poisson map. When the action is also transitive, $(M, \pi)$ is called a $\left(U, \pi_{U}\right)$-homogeneous Poisson space. In this case, Drinfeld [6] associated to each $m \in M$ a Lagrangian subalgebra $\mathfrak{l}_{m}$ of $\mathfrak{d}$ and showed that $\mathfrak{l}_{u \cdot m}=\operatorname{Ad}_{u} \mathfrak{l}_{m}$ for every $u \in U$ and $m \in M$. Thus we have a $U$-equivariant map

$$
P: M \longrightarrow \mathcal{L}(\mathfrak{d}): m \longmapsto \mathfrak{l}_{m}
$$

where $U$ acts on $\mathcal{L}(\mathfrak{d})$ by the adjoint action. Drinfeld's theorem says that the assignment that assigns to each $(M, \pi)$ the image of the map $P$ in (1) gives a one-to-one correspondence between the set of $U$-equivariant isomorphism classes of $\left(U, \pi_{U}\right)$-homogeneous Poisson spaces with connected stabilizer subgroups and the set of $U$-orbits in a certain subset $\mathcal{L}(\mathfrak{d})_{C}$ of $\mathcal{L}(\mathfrak{d})$ (see Section 2.1 for more details).

We prove the following theorem.

THEOREM 1.1. - 1) There is a Poisson structure $\Pi$ on $\mathcal{L}(\mathfrak{d})$ with respect to which the adjoint action of $U$ on $\mathcal{L}(\mathfrak{d})$ is Poisson.

2) Each $U$-orbit $\mathcal{O}$ in $\mathcal{L}(\mathfrak{d})$ is a Poisson submanifold and consequently a $\left(U, \pi_{U}\right)$-homogeneous Poisson space.

3) For any $\left(U, \pi_{U}\right)$-homogeneous Poisson space $(M, \pi)$, the map $P$ in (1) is a Poisson map onto the $U$-orbit of $\mathfrak{l}_{m}$ for any $m \in M$.

We introduce the notion of model points in $\mathcal{L}(\mathfrak{d})$. For a homogeneous Poisson space $(M, \pi)$, let $\mathfrak{l}=P(m)$ for some $m \in M$. We show $\mathfrak{l}$ is a model point if and only if the map $P: M \rightarrow \mathcal{O}_{\mathfrak{l}}=U \cdot \mathfrak{l}$ is a local diffeomorphism (and thus a covering map). When this happens, we regard $\left(\mathcal{O}_{\mathfrak{l}}, \Pi\right)$ as a model for the Poisson space $(M, \pi)$.

The second part of the paper is concerned with the variety $\mathcal{L}$ of Lagrangian subalgebras of a complex semi-simple Lie algebra $\mathfrak{g}$ with respect to the imaginary part of its Killing form. Let $G$ be the adjoint group of $\mathfrak{g}$. Based on the Karolinsky classification of Lagrangian subalgebras of $\mathfrak{g}$ in [12], we prove:

THEOREM 1.2. - The irreducible components of $\mathcal{L}$ are smooth. Each irreducible component fibers over a generalized flag variety, and its fiber is the product of a connected component of a real orthogonal group and the space of real points of a De Concini-Procesi compactification of the semi-simple part of a Levi subgroup of $G$.

For example, when $\mathfrak{g}=\mathfrak{s l}(2, \mathbb{C})$, there are two irreducible components: the first component is the $\mathrm{SL}(2, \mathbb{C})$-orbit through $\mathfrak{a}+\mathfrak{n}$ and is isomorphic to $\mathbb{C} \mathrm{P}^{1}$ (here $\mathfrak{a}$ consists of diagonal real trace zero matrices and $\mathfrak{n}$ strictly upper triangular matrices), and the second component contains the 
$\mathrm{SL}(2, \mathbb{C})$-orbits through $\mathfrak{s u}(2)$ and $\mathfrak{s l}(2, \mathbb{R})$ as open orbits, and the $\mathrm{SL}(2, \mathbb{C})$-orbit through $i \mathfrak{a}+\mathfrak{n}$ as the unique closed orbit. The second component may be identified as $\mathbb{R P}^{3}$.

Let $\mathfrak{k}$ be a compact real form of $\mathfrak{g}$ and $K \subset G$ the connected subgroup with Lie algebra $\mathfrak{k}$. Fix an Iwasawa decomposition $\mathfrak{g}=\mathfrak{k}+\mathfrak{a}+\mathfrak{n}$ of $\mathfrak{g}$. Then there is a Poisson structure $\pi_{K}$ on $K$ making $\left(K, \pi_{K}\right)$ into a Poisson Lie group, and the double of the tangent Lie bialgebra of $\left(K, \pi_{K}\right)$ is $\mathfrak{g}$. By Theorem 1.1, there is a Poisson structure $\Pi$ on $\mathcal{L}$ such that each $K$-orbit in $\mathcal{L}$, being a Poisson submanifold of $(\mathcal{L}, \Pi)$, is a $\left(K, \pi_{K}\right)$-homogeneous Poisson space, and every $\left(K, \pi_{K}\right)$ homogeneous Poisson space maps onto a $K$-orbit in $\mathcal{L}$ by a Poisson map. In particular, we show that every point in the (unique) irreducible component $\mathcal{L}_{0}$ of $\mathcal{L}$ that contains $\mathfrak{k}$ is a model point. Consequently, a number of interesting $\left(K, \pi_{K}\right)$-homogeneous Poisson spaces are contained in $\mathcal{L}_{0}$ (possibly up to covering maps) as Poisson submanifolds. Among these are all $\left(K, \pi_{K}\right)$-homogeneous Poisson structures on any $K / K_{1}$, where $K_{1}$ is a closed subgroup of $K$ containing a maximal torus of $K$. For example, $K / K_{1}$ could be any flag variety $G / Q \cong K / K \cap Q$, where $Q$ is a parabolic subgroup of $G$. We remark that it is shown in [21] that all $\left(K, \pi_{K}\right)$-homogeneous Poisson structures on $K / T$, where $T$ is a maximal torus in $K$, can be obtained from solutions to the Classical Dynamical Yang-Baxter Equation [8]. Some Poisson geometrical properties of such Poisson structures are also studied in [21].

We are motivated to study $\left(K, \pi_{K}\right)$-homogeneous Poisson structures because of their connections to Lie theory. One remarkable example is the so-called Bruhat Poisson structure $\pi_{\infty}$ [17] on $K / T$. It corresponds to the Lagrangian subalgebra $\mathfrak{t}+\mathfrak{n}$ of $\mathfrak{g}$, where $\mathfrak{g}=\mathfrak{k}+\mathfrak{a}+\mathfrak{n}$ is an Iwasawa decomposition of $\mathfrak{g}$, and $\mathfrak{t}=i \mathfrak{a}$ is the Lie algebra of $T$. The name Bruhat Poisson structure comes from the fact that its symplectic leaves are exactly the Bruhat cells for a Bruhat decomposition of $K / T$ [17]; its Poisson cohomology is isomorphic to a direct sum of $\mathfrak{n}$-cohomology groups with coefficients in certain infinite-dimensional representations of $G$ [19]; its $K$-invariant Poisson harmonic forms are exactly the harmonic forms introduced and studied by Kostant in [15]. This last fact is proved in [7], where we also use $\pi_{\infty}$ to construct $\mathrm{S}^{1}$-equivariantly closed forms on $K / T$ and use them to reinterpret the Kostant-Kumar approach to the Schubert calculus on $K / T$ [16]. One key fact used in [7] is that the Poisson structure $\pi_{\infty}$ is the limit of a family $\pi_{t}, t \in(0,+\infty)$, of $\left(K, \pi_{K}\right)$-homogeneous symplectic structures on $K / T$. The family $\pi_{t}, t \in(0,+\infty]$, corresponds to a continuous curve in $\mathcal{L}$. Thus, we regard $\mathcal{L}$ as a natural setting for deformation problems for Poisson homogeneous spaces, and for this reason it is desirable to study its geometry.

The paper is organized as follows.

We start our discussion in Section 2 with an arbitrary Poisson Lie group $\left(U, \pi_{U}\right)$, its tangent Lie bialgebra $\left(\mathfrak{u}, \mathfrak{u}^{*}\right)$, and the variety $\mathcal{L}(\mathfrak{d})$ of Lagrangian subalgebras of its double $\mathfrak{d}=\mathfrak{u} \bowtie \mathfrak{u}^{*}$. We first review Drinfeld's theorem on $\left(U, \pi_{U}\right)$-homogeneous spaces. We then give the construction of the Poisson structure $\Pi$ on $\mathcal{L}(\mathfrak{d})$ and establish the properties listed in Theorem 1.1.

The rest of the paper is devoted to the Poisson Lie group $\left(K, \pi_{K}\right)$. In $\S 3.1$, we review Karolinsky's classification of Lagrangian subalgebras, and use it to decompose $\mathcal{L}$ into a finite disjoint union of submanifolds $\mathcal{L}(S, \varepsilon, d)$. The study of the closure $\mathcal{L}(S, \varepsilon, d)$ is reduced to studying the closure of the variety of real forms of a semi-simple Lie algebra. After some preliminary results in Section 4, we identify the closure with the set of real points of a De Concini-Procesi compactification in Section 5. In Section 6, we apply our results to determine the irreducible components of $\mathcal{L}$ and show they are smooth. We also study the set of model points in $\mathcal{L}$ and show that every Lie algebra in the irreducible component $\mathcal{L}_{0}$ containing $\mathfrak{k}$ is a model point. In Section 7, we study some properties of the Poisson structure $\Pi$. In particular, we study the $K$-orbits in the irreducible component $\mathcal{L}_{0}$ and the $\left(K, \pi_{K}\right)$-homogeneous Poisson spaces arising from them. In the last section, we discuss relations with other recent work and some unresolved problems for a sequel to this paper. 


\section{Generalities on Lie bialgebras}

\subsection{Drinfeld's theorem}

In this section, we review Drinfeld's theorem on homogeneous spaces of Poisson Lie groups in [6]. Details on Poisson Lie groups can be found in [17] and [14] and the references cited in [14].

Let $\left(U, \pi_{U}\right)$ be a Poisson Lie group with tangent Lie bialgebra $\left(\mathfrak{u}, \mathfrak{u}^{*}\right)$, where $\mathfrak{u}$ is the Lie algebra of $U$ and $\mathfrak{u}^{*}$ its dual space equipped with a Lie algebra structure coming from the linearization of $\pi_{U}$ at the identity element of $U$. We will use letters $x, y, x_{1}, y_{1}, \ldots$ to denote elements in $\mathfrak{u}$ and $\xi, \eta, \xi_{1}, \eta_{1}, \ldots$ for elements in $\mathfrak{u}^{*}$. The pairing between elements in $\mathfrak{u}$ and in $\mathfrak{u}^{*}$ will be denoted by $($,$) .$

Let $\langle$,$\rangle be the symmetric non-degenerate scalar product on the direct sum vector space \mathfrak{u} \oplus \mathfrak{u}^{*}$ defined by

$$
\left\langle x_{1}+\xi_{1}, x_{2}+\xi_{2}\right\rangle=\left(x_{1}, \xi_{2}\right)+\left(x_{2}, \xi_{1}\right) .
$$

Then there is a unique Lie bracket on the $\mathfrak{u} \oplus \mathfrak{u}^{*}$ such that $\langle$,$\rangle is ad-invariant and that both \mathfrak{u}$ and $\mathfrak{u}^{*}$ are its Lie subalgebras with respect to the natural inclusions. The vector space $\mathfrak{u} \oplus \mathfrak{u}^{*}$ together with this Lie bracket is called the double Lie algebra of $\left(\mathfrak{u}, \mathfrak{u}^{*}\right)$ and we will denote it by $\mathfrak{d}=\mathfrak{u} \bowtie \mathfrak{u}^{*}$. Note that $U$ acts on $\mathfrak{d}$ by the adjoint action (by first mapping $U$ to the adjoint group of $\mathfrak{d})$.

Example 2.1. - Let $\mathfrak{u}=\mathfrak{k}$ be a compact semi-simple Lie algebra. Let $\mathfrak{g}=\mathfrak{k}_{\mathbb{C}}$ be the complexification of $\mathfrak{k}$ with an Iwasawa decomposition $\mathfrak{g}=\mathfrak{k}+\mathfrak{a}+\mathfrak{n}$. Let $\langle$,$\rangle be twice the$ imaginary part of the Killing form of $\mathfrak{g}$. Then the pairing between $\mathfrak{k}$ and $\mathfrak{a}+\mathfrak{n}$ via $\langle$,$\rangle gives an$ identification of $\mathfrak{k}^{*}$ and $\mathfrak{a}+\mathfrak{n}$, and $(\mathfrak{k}, \mathfrak{a}+\mathfrak{n})$ becomes a Lie bialgebra whose double is $\mathfrak{g}$. If $K$ is any group with Lie algebra $\mathfrak{k}$, then there is a Poisson structure $\pi_{K}$ on $K$ making $\left(K, \pi_{K}\right)$ into a Poisson Lie group whose tangent Lie bialgebra is $(\mathfrak{k}, \mathfrak{a}+\mathfrak{n})$. This will be our most important example.

DEFINITION 2.2. - Let $n=\operatorname{dim} \mathfrak{u}$. A Lie subalgebra $\mathfrak{l}$ of $\mathfrak{d}$ is called Lagrangian if $\langle a, b\rangle=0$ for all $a, b \in \mathfrak{l}$ and if $\operatorname{dim} \mathfrak{l}=n$. The set of all Lagrangian subalgebras of $\mathfrak{d}$ will be denoted by $\mathcal{L}(\mathfrak{d})$.

Both $\mathfrak{u}$ and $\mathfrak{u}^{*}$ are Lagrangian. If $D$ is the adjoint group of $\mathfrak{d}$, then $D$ acts on the set of Lagrangian subalgebras. In Example 2.1, any real form of $\mathfrak{g}$ is a Lagrangian subalgebra, as is $\mathfrak{t}+\mathfrak{n}$, where $\mathfrak{t}=i \mathfrak{a}$ is the centralizer of $\mathfrak{a}$ in $\mathfrak{k}$.

Let $(M, \pi)$ be a $\left(U, \pi_{U}\right)$-homogeneous Poisson space. Recall [6] that this means that $U$ acts on $M$ transitively and that the action map $U \times M \rightarrow M$ is a Poisson map, where $U \times M$ is equipped with the direct product Poisson structure $\pi_{U} \oplus \pi$. Let $m \in M$. Then, since the Poisson structure $\pi$ on $M$ is $\left(U, \pi_{U}\right)$-homogeneous, it must satisfy

$$
\pi(u m)=u_{*} \pi(m)+m_{*} \pi_{U}(u), \quad \forall u \in U, m \in M .
$$

Here $u_{*}$ and $m_{*}$ are respectively the differentials of the maps $M \rightarrow M: m_{1} \mapsto u m_{1}$ and $U \rightarrow M: u_{1} \mapsto u_{1} m$. Thus, $\pi$ is totally determined by its value $\pi(m) \in \wedge^{2}\left(\mathrm{~T}_{m} M\right)$ at $m$. Let $U_{m} \subset U$ be the stabilizer subgroup of $U$ at $m$ with Lie algebra $\mathfrak{u}_{m}$. Identify $\mathrm{T}_{m} M \cong \mathfrak{u} / \mathfrak{u}_{m}$ so that $\pi(m) \in \wedge^{2}\left(\mathfrak{u} / \mathfrak{u}_{m}\right)$. Let $\mathfrak{l}_{m}$ be the subspace of $\mathfrak{d}$ defined by

$$
\left.\mathfrak{l}_{m}=\left\{x+\xi: x \in \mathfrak{u}, \xi \in \mathfrak{u}^{*},\left.\xi\right|_{\mathfrak{u}_{m}}=0, \xi\right\lrcorner \pi(m)=x+\mathfrak{u}_{m}\right\} .
$$


THEOREM 2.3 (Drinfeld [6]). - 1) $\mathfrak{l}_{m}$ is a Lagrangian subalgebra of $\mathfrak{d}$ for all $m \in M$.

2) For all $m \in M$ and $u \in U$,

$$
\begin{aligned}
\mathfrak{l}_{m} \cap \mathfrak{u} & =\mathfrak{u}_{m}, \\
\operatorname{Ad}_{u} \mathfrak{l}_{m} & =\mathfrak{l}_{u m}, \quad \forall u \in U .
\end{aligned}
$$

3) Let $M$ be a $U$-homogeneous space. A $\left(U, \pi_{U}\right)$-homogeneous Poisson structure $\pi$ on $M$ is equivalent to a $U$-equivariant map $\mathrm{P}: M \rightarrow \mathcal{L}(\mathfrak{d}): m \mapsto \mathfrak{l}_{m}$ such that (5) holds for all $m \in M$.

DEFINITION 2.4. - We will call $\mathfrak{l}_{m}$ the Lagrangian subalgebra of $\mathfrak{d}$ associated to $(M, \pi)$ at the point $m$. The map $\mathrm{P}: M \rightarrow \mathcal{L}(\mathfrak{d})$ will be called the Drinfeld map.

DEFINITION 2.5. - Given a $U$-homogeneous space $M$, we say that a $U$-equivariant map $M \rightarrow \mathcal{L}(\mathfrak{d}): m \mapsto \mathfrak{l}_{m}$ has Property I (I for intersection) if (5) is satisfied for all $m \in M$.

Thus 3) of Theorem 2.3 can be rephrased as follows: given a $U$-homogeneous space $M$, a $\left(U, \pi_{U}\right)$-homogeneous Poisson structure on $M$ is equivalent to a $U$-equivariant map $M \rightarrow \mathcal{L}(\mathfrak{d})$ with Property I.

Remark 2.6. - We explain how a $U$-equivariant map $M \rightarrow \mathcal{L}(\mathfrak{d})$ having Property I gives a $\left(U, \pi_{U}\right)$-homogeneous Poisson structure on $M$ : pick any $m \in M$. Because $\mathfrak{l}_{m} \subset \mathfrak{d}$ is maximal isotropic (this means that $\operatorname{dim} \mathfrak{l}_{m}=n$ and that $\langle a, b\rangle=0$ for all $a, b \in \mathfrak{l}_{m}$ ) and because of (5), an easy linear algebra argument (see also Lemma 2.24) shows that there is a unique element $\pi(m) \in \wedge^{2}\left(\mathfrak{u} / \mathfrak{u}_{m}\right)$ such that (4) holds. Define a bivector field $\pi$ on $M$ by (3). This is well defined because of (6). This $\pi$ is Poisson because $\mathfrak{l}_{m}$ is Lagrangian. It is $\left(U, \pi_{U}\right)$-homogeneous because (3) holds by definition.

We now state some consequences of Theorem 2.3.

Definition 2.7. - A Lagrangian subalgebra of $\mathfrak{d}$ is said to have Property $C$ ( $\mathrm{C}$ for closed) if the connected subgroup $U_{\mathfrak{l}}^{\prime}$ of $U$ with Lie algebra $\mathfrak{l} \cap \mathfrak{u}$ is closed in $U$.

Note that any $\mathfrak{l}_{m}$ in the image of the Drinfeld map for any $(M, \pi)$ has Property $\mathrm{C}$, because the connected subgroup of $U$ with Lie algebra $\mathfrak{l}_{m} \cap \mathfrak{u}$ is the identity connected component of the stabilizer subgroup of $U$ at $m$, so it is closed in $U$. Conversely, if $\mathfrak{l} \in \mathcal{L}(\mathfrak{d})$ has Property C, we have the $U$-homogeneous space $U / U_{\mathfrak{l}}^{\prime}$ and the $U$-equivariant map

$$
U / U_{\mathfrak{l}}^{\prime} \longrightarrow \mathcal{L}(\mathfrak{d}): u U_{\mathfrak{l}}^{\prime} \longmapsto \operatorname{Ad}_{u} \mathfrak{l}
$$

It has Property I. More generally, suppose that $U_{1}$ is any closed subgroup of $U$ having the properties:

(A) the Lie algebra of $U_{1}$ is $\mathfrak{l} \cap \mathfrak{u}$;

(B) $U_{1}$ normalizes $\mathfrak{l}$,

Then we have the $U$-equivariant map

$$
U / U_{1} \longrightarrow \mathcal{L}(\mathfrak{d}): u U_{1} \longmapsto \operatorname{Ad}_{u} \mathfrak{l}
$$

It has Property I. Thus, by Theorem 2.3, we have:

COROLlaRY 2.8. - Suppose that $\mathfrak{l} \in \mathcal{L}(\mathfrak{d})$ has Property $\mathrm{C}$. Then, for any closed subgroup $U_{1}$ of $U$ having Properties (A) and (B), there is a $\left(U, \pi_{U}\right)$-homogeneous Poisson structure on $U / U_{1}$ whose Drinfeld map is given by

$$
\mathrm{P}: U / U_{1} \longrightarrow \mathcal{L}(\mathfrak{d}): u U_{1} \longmapsto \operatorname{Ad}_{u} \mathfrak{l} .
$$


DeFinition 2.9. - For a Lagrangian subalgebra $\mathfrak{l}$ of $\mathfrak{d}$ with Property $\mathrm{C}$ and any subgroup $U_{1}$ of $U$ with the above Properties (A) and (B), we say that the Poisson manifold $\left(U / U_{1}, \pi\right)$ described in Corollary 2.8 is determined by $\mathfrak{l}$.

Denote by $\mathcal{L}(\mathfrak{d})_{C}$ the set of all points in $\mathcal{L}(\mathfrak{d})$ with Property $\mathrm{C}$. It is clearly invariant under the adjoint action of $U$. For every $\left(U, \pi_{U}\right)$-homogeneous Poisson space $(M, \pi)$, the image of the Drinfeld map $M \rightarrow \mathcal{L}(\mathfrak{d})$ is a $U$-orbit in $\mathcal{L}(\mathfrak{d})_{C}$.

COROLlary 2.10 (Drinfeld [6]). - The map that assigns to each $(M, \pi)$ the image of its Drinfeld map gives a one-to-one correspondence between $U$-equivariant isomorphism classes of $\left(U, \pi_{U}\right)$-homogeneous Poisson spaces with connected stabilizer subgroups and the set of $U$-orbits in $\mathcal{L}(\mathfrak{d})_{C}$.

We close this section by an example of a Lagrangian subalgebra $\mathfrak{l}$ that does not have Property C.

Example 2.11 ([12]). - Consider the Lie bialgebra $(\mathfrak{k}, \mathfrak{a}+\mathfrak{n})$ in Example 2.1. Let $U=K$ be a compact connected Lie group with Lie algebra $\mathfrak{k}$ and let $T$ be the maximal torus of $K$ with Lie algebra $i \mathfrak{a}$. Choose a topological generator $t$ of $T$ and let $t=\exp (X), X \in \mathfrak{t}$. Let $\mathfrak{l}=\mathbb{R} \cdot X+\left(\mathfrak{a} \cap(\mathbb{R} \cdot X)^{\perp}\right)+\mathfrak{n}$, where the perpendicular is computed relative to the Killing form. Then $\mathfrak{l}$ is Lagrangian, but if $\operatorname{rank}(T)>1$ then $\mathfrak{l} \cap \mathfrak{k}$ is not the Lie algebra of a closed subgroup of $K$, so $\mathfrak{l}$ does not have Property $\mathrm{C}$.

\subsection{A Poisson structure on $\mathcal{L}(\mathfrak{d})$}

Let $\left(U, \pi_{U}\right)$ be a Poisson Lie group and let $\left(\mathfrak{u}, \mathfrak{u}^{*}\right)$ be its tangent Lie bialgebra. Let $\mathfrak{d}=\mathfrak{u} \bowtie \mathfrak{u}^{*}$ be its double Lie algebra equipped with the symmetric scalar product $\langle$,$\rangle given by (2). Recall$ that $\mathcal{L}(\mathfrak{d})$ is the set of Lagrangian subalgebras of $\mathfrak{d}$ with respect to $\langle$,$\rangle .$

Notation 2.12. - We will use $\operatorname{Gr}(n, \mathfrak{d})$ to denote the Grassmannian of $n$-dimensional subspaces of $\mathfrak{d}$. Since the condition of being closed under Lie bracket and the condition of being Lagrangian are polynomial conditions, $\mathcal{L}(\mathfrak{d}) \subset \operatorname{Gr}(n, \mathfrak{d})$ is an algebraic subset.

The group $U$ acts on $\operatorname{Gr}(n, \mathfrak{d})$ by the adjoint action and it leaves $\mathcal{L}(\mathfrak{d})$ invariant. Although $\mathcal{L}(\mathfrak{d})$ may be singular, all the $U$-orbits in $\mathcal{L}(\mathfrak{d})$ are smooth.

In this section, we will show that there is a smooth bi-vector field $\Pi$ on $\operatorname{Gr}(n, \mathfrak{d})$ with the property

$$
[\Pi, \Pi](\mathfrak{l})=0
$$

for every $\mathfrak{l} \in \mathcal{L}(\mathfrak{d})$, where $[\Pi, \Pi]$ is the Schouten bracket of $\Pi$ with itself. Moreover, we show that $\Pi$ is tangent to every $U$-orbit $\mathcal{O}$ in $\mathcal{L}(\mathfrak{d})$, so $(\mathcal{O}, \Pi)$ is a Poisson manifold. In fact, each $(\mathcal{O}, \Pi)$ is a $\left(U, \pi_{U}\right)$-homogeneous Poisson space. If $(M, \pi)$ is a $\left(U, \pi_{U}\right)$-homogeneous Poisson space, we show that the Drinfeld map P : $M \rightarrow \mathcal{O}$ is a Poisson map, where $\mathcal{O}$ is the $U$-orbit of $\mathfrak{l}_{m}$ for any $m \in M$.

Notation 2.13. - We identify $\mathfrak{d}^{*} \cong \mathfrak{u}^{*} \oplus \mathfrak{u}$ in the obvious way. Denote by $\#: \mathfrak{d}^{*} \rightarrow \mathfrak{d}$ the isomorphism induced by the nondegenerate pairing $\langle$,$\rangle on \mathfrak{d}$. It is given by

$$
\#: \mathfrak{d}^{*} \longrightarrow \mathfrak{d}: \#(\xi+x)=x+\xi
$$

For $V \subset \mathfrak{d}$, we let

$$
V^{\circ}=\left\{f \in \mathfrak{d}^{*}:\left.f\right|_{V}=0\right\}
$$


To define the bi-vector field $\Pi$ on $\operatorname{Gr}(n, \mathfrak{d})$, we consider the element $R \in \wedge^{2} \mathfrak{d}$ defined by

$$
R\left(\xi_{1}+x_{1}, \xi_{2}+x_{2}\right)=\left(\xi_{2}, x_{1}\right)-\left(\xi_{1}, x_{2}\right), \quad \forall x_{1}, x_{2} \in \mathfrak{u}, \xi_{1}, \xi_{2} \in \mathfrak{u}^{*}
$$

The element $R$ is an example of a classical $r$-matrix on $\mathfrak{d}$ [14]. In particular, the Schouten bracket $[R, R] \in \wedge^{3} \mathfrak{d}$ of $R$ with itself is ad-invariant and is given by

$$
[R, R]\left(f_{1}, f_{2}, f_{3}\right)=2\left\langle \# f_{1},\left[\# f_{2}, \# f_{3}\right]\right\rangle
$$

for $f_{i} \in \mathfrak{d}^{*}$. Denote by $\chi^{k}(\operatorname{Gr}(n, \mathfrak{d}))$ the space of $k$-vector fields on $\operatorname{Gr}(n, \mathfrak{d})$ (i.e., the space of smooth sections of the $k$ th exterior power of the tangent bundle of $\operatorname{Gr}(n, \mathfrak{d}))$. The action by the adjoint group $D$ of $\mathfrak{d}$ on $\operatorname{Gr}(n, \mathfrak{d})$ gives a Lie algebra anti-homomorphism

$$
\kappa: \mathfrak{d} \longrightarrow \chi^{1}(\operatorname{Gr}(n, \mathfrak{d}))
$$

whose multi-linear extension from $\wedge^{k} \mathfrak{d}$ to $\chi^{k}(\operatorname{Gr}(n, \mathfrak{d}))$, for any integer $k \geqslant 1$, will also be denoted by $\kappa$.

Define the bi-vector field $\Pi$ on $\operatorname{Gr}(n, \mathfrak{d})$ by

$$
\Pi=\frac{1}{2} \kappa(R) .
$$

THEOREM 2.14. - For every Lagrangian subalgebra $\mathfrak{l}$ of $\mathfrak{d}$ regarded as a point in $\operatorname{Gr}(n, \mathfrak{d})$, we have

$$
[\Pi, \Pi](\mathfrak{l})=0,
$$

where $[\Pi, \Pi]$ is the Schouten bracket of $\Pi$ with itself.

Proof. - Since $\Pi=\frac{1}{2} \kappa(R)$ and since $\kappa$ is a Lie algebra anti-homomorphism, we have

$$
[\Pi, \Pi]=-\frac{1}{4} \kappa([R, R])
$$

Let $D_{\mathfrak{l}}$ be the stabilizer subgroup of $D$ at $\mathfrak{l}$ for the adjoint action, and let $\mathfrak{d}_{\mathfrak{l}}$ be its Lie algebra. Since $\Pi$ is tangent to the $D$-orbit $D \cdot \mathfrak{l}$ in $\operatorname{Gr}(n, \mathfrak{d})$, we only need to show that $[\Pi, \Pi]=0$ when evaluated on a triple $\left(\alpha_{1}, \alpha_{2}, \alpha_{3}\right)$ of covectors in $\mathrm{T}_{\mathfrak{l}}^{*}(D \cdot \mathfrak{l})$. The map

$$
\kappa: \mathfrak{d} \longrightarrow \mathrm{T}_{\mathfrak{l}}(D \cdot \mathfrak{l})
$$

gives an identification

$$
\kappa^{*}: \mathrm{T}_{\mathfrak{l}}^{*}(D \cdot \mathfrak{l}) \longrightarrow \mathfrak{d}_{\mathfrak{l}}^{\circ}
$$

Thus, it suffices to show

$$
[R, R]\left(f_{1}, f_{2}, f_{3}\right)=0
$$

for $f_{i} \in \mathfrak{d}_{\mathfrak{l}}^{\circ}, i=1,2,3$. Since $\mathfrak{l} \subset \mathfrak{d}_{\mathfrak{l}}$, we have $\#\left(\mathfrak{d}_{\mathfrak{l}}^{\circ}\right) \subset \#\left(\mathfrak{l}^{\circ}\right)=\mathfrak{l}$. It follows that

$$
[R, R]\left(f_{1}, f_{2}, f_{3}\right)=2\left\langle \# f_{1},\left[\# f_{2}, \# f_{3}\right]\right\rangle=0
$$

because $\mathfrak{l}$ is a Lagrangian subalgebra. 
If $X$ is an algebraic variety, denote by $A_{X}$ the sheaf of algebraic functions on $X$, and if $Y \subset X$ is a closed subvariety, denote by $I_{Y} \subset A_{X}$ the sheaf of functions vanishing on $Y$. For locally defined functions $f$ and $g$ in $A_{\mathrm{Gr}(n, \mathfrak{d})}$, let $\{f, g\}=\Pi(\mathrm{d} f, \mathrm{~d} g) \in A_{\mathrm{Gr}(n, \mathfrak{d})}$.

COROLlary 2.15.- $(\mathcal{L}(\mathfrak{d}),\{\cdot, \cdot\})$ is a Poisson algebraic variety.

Proof. - We have to check that $\{\cdot, \cdot\}$ induces the structure of a Poisson algebra on $A_{\mathcal{L}(\mathfrak{d})}$. For $f \in A_{\mathrm{Gr}(n, \mathfrak{d})}$, let $\xi_{f}$ be the local vector field defined by $\xi_{f}(g)=\{f, g\}$, where $g \in A_{\mathrm{Gr}(n, \mathfrak{d})}$. It follows easily from the definition that if $g \in I_{\mathcal{L}(\mathfrak{d})}$, then $\xi_{f}(g)$ vanishes on $\mathcal{L}(\mathfrak{d})$, i.e., $\xi_{f}$ is tangent to $\mathcal{L}(\mathfrak{d})$. It follows that the bracket $\{\cdot, \cdot\}$ descends from $A_{\mathrm{Gr}(n, \mathfrak{d})}$ to $A_{\mathcal{L}(\mathfrak{d})}$. Although $\{\cdot, \cdot\}$ does not define a Poisson algebra structure on $A_{\mathrm{Gr}(n, \mathfrak{d})}$, Theorem 2.14 implies that it does define a Poisson algebra structure on $A_{\mathcal{L}(\mathfrak{d})}$.

COROLlary 2.16. - For every $\mathfrak{l} \in \mathcal{L}(\mathfrak{d}) \subset \operatorname{Gr}(n, \mathfrak{d})$, the bivector field $\Pi$ defines a Poisson structure on the D-orbit $D \cdot \mathfrak{l}$ in $\operatorname{Gr}(n, \mathfrak{d})$.

Since $[R, R] \in \wedge^{3} \mathfrak{d}$ is ad-invariant, the following bivector field $\pi_{-}$on $D$ is Poisson:

$$
\pi_{-}(d)=\frac{1}{2}\left(r_{d} R-l_{d} R\right), \quad d \in D,
$$

where $r_{d}$ and $l_{d}$ are respectively the differentials of the right and left translations on $D$ defined by $d$. Moreover, $\left(D, \pi_{-}\right)$is a Poisson Lie group and $\left(U, \pi_{U}\right)$ is a Poisson subgroup of $\left(D, \pi_{-}\right)$ (see [18]).

Proposition 2.17. - For every $\mathfrak{l} \in \mathcal{L}(\mathfrak{d})$, the Poisson manifold $(D \cdot \mathfrak{l}, \Pi)$ is $\left(D, \pi_{-}\right)$-homogeneous.

Proof. - Let again $D_{\mathfrak{l}}$ be the stabilizer subgroup of $\mathfrak{l}$ in $D$. Then $D \cdot \mathfrak{l} \cong D / D_{\mathfrak{l}}$. Consider the bivector field $\Pi_{1}$ on $D$ defined by

$$
\Pi_{1}(d)=\frac{1}{2} r_{d} R, \quad d \in D .
$$

Then $\Pi=p_{*} \Pi_{1}$, where $p: D \rightarrow D / D_{\mathfrak{l}}$ is the natural projection and $p_{*}$ its differential. It is easy to check that, for any $d_{1}, d_{2} \in D$, we have

$$
\Pi_{1}\left(d_{1} d_{2}\right)=l_{d_{1}} \Pi_{1}\left(d_{2}\right)+r_{d_{2}} \pi_{-}\left(d_{1}\right) .
$$

It follows that $(D \cdot \mathfrak{l}, \Pi)$ is a $\left(D, \pi_{-}\right)$-homogeneous Poisson space.

Consider now the $U$-orbits in $\mathcal{L}(\mathfrak{d})$ through a point $\mathfrak{l} \in \mathcal{L}(\mathfrak{d})$. We have:

THEOREM 2.18. - At any $\mathfrak{l} \in \mathcal{L}(\mathfrak{d})$, the bi-vector field $\Pi$ on $\operatorname{Gr}(n, \mathfrak{d})$ is tangent to the $U$-orbit through $\mathfrak{l}$, so that $(U \cdot \mathfrak{l}, \Pi)$ is a Poisson submanifold of $(D \cdot \mathfrak{l}, \Pi)$.

Proof. - Regard $\Pi$ as a bivector field on the $D$-orbit $D \cdot \mathfrak{l}$, so $\Pi(\mathfrak{l}) \in \wedge^{2} \mathrm{~T}_{\mathfrak{l}}(D \cdot \mathfrak{l})$. Let $\Pi(\mathfrak{l})^{\#}$ be the linear map

$$
\begin{aligned}
\Pi(\mathfrak{l})^{\#}: & \mathrm{T}_{\mathfrak{l}}^{*}(D \cdot \mathfrak{l}) \longrightarrow \mathrm{T}_{\mathfrak{l}}(D \cdot \mathfrak{l}): \\
& \Pi(\mathfrak{l})^{\#}(\alpha)(\beta)=\Pi(\mathfrak{l})(\alpha, \beta), \quad \alpha, \beta \in \mathrm{T}_{\mathfrak{l}}^{*}(D \cdot \mathfrak{l}) .
\end{aligned}
$$

It is enough to show that the image of $\Pi(\mathfrak{l})^{\#}$ is tangent to the $U$-orbit through $\mathfrak{l}$. 
By the identification, $T_{\mathfrak{l}}^{*}(D \cdot \mathfrak{l}) \rightarrow \mathfrak{d}_{\mathfrak{l}}^{\circ}$, it is enough to show that

$$
\kappa((\xi+x)\lrcorner R) \in \mathrm{T}_{\mathfrak{l}}(U \cdot \mathfrak{l}), \quad \forall \xi+x \in \mathfrak{d}_{\mathfrak{l}}^{\circ},
$$

where $(\xi+x)\lrcorner R \in \mathfrak{d}$ is defined by

$$
((\xi+x)\lrcorner R)(\eta+y)=R(\xi+x, \eta+y), \quad \forall \eta+y \in \mathfrak{d}^{*} .
$$

We compute explicitly. It follows from the definition of $R$ that

$$
R=\sum_{i=1}^{n} \eta_{i} \wedge e_{i} \in \wedge^{2} \mathfrak{d}
$$

where $\left\{e_{1}, \ldots, e_{n}\right\}$ is a basis for $\mathfrak{u}$ and $\left\{\eta_{1}, \ldots, \eta_{n}\right\}$ is its dual basis for $\mathfrak{u}^{*}$. It follows that

$$
(\xi+x)\lrcorner R=\sum_{i=1}^{n}\left(\left(x, \eta_{i}\right) e_{i}-\left(\xi, e_{i}\right) \eta_{i}\right)=x-\xi .
$$

Hence

$$
\kappa((\xi+x)\lrcorner R)=\kappa(x)-\kappa(\xi) .
$$

But since $\xi+x \in \mathfrak{d}_{\mathfrak{l}}^{\circ}$, we have $x+\xi \in \mathfrak{l}$, so $\kappa(x+\xi)=0$. Thus

$$
\kappa((\xi+x)\lrcorner R)=2 \kappa(x) \in \mathrm{T}_{\mathfrak{l}}(U \cdot \mathfrak{l}) .
$$

Corollary 2.19. - For every $\mathfrak{l} \in \mathcal{L}(\mathfrak{d})$, the Poisson manifold $(U \cdot \mathfrak{l}, \Pi)$ is a $\left(U, \pi_{U}\right)$-homogeneous Poisson space.

Proof. - This follows from Proposition 2.17 because $\left(U, \pi_{U}\right)$ is a Poisson subgroup of $\left(D, \pi_{-}\right)$ and $(U \cdot \mathfrak{l}, \Pi)$ is a Poisson submanifold of $(D \cdot \mathfrak{l}, \Pi)$.

Remark 2.20. - Let $U^{*}$ be the connected and simply connected group with Lie algebra $\mathfrak{u}^{*}$. Then for any Lagrangian subalgebra $\mathfrak{l} \in \mathcal{L}(\mathfrak{d})$, the orbit $U^{*} \cdot \mathfrak{l}$ is also a Poisson submanifold of $(D \cdot \mathfrak{l}, \Pi)$. Indeed, the roles of $\mathfrak{u}$ and $\mathfrak{u}^{*}$ are symmetric in the definition of $D$ and of $\mathcal{L}(\mathfrak{d})$, but the $R$-matrix for the Lie bialgebra $\left(\mathfrak{u}^{*}, \mathfrak{u}\right)$ differs from that for $\left(\mathfrak{u}, \mathfrak{u}^{*}\right)$ by a minus sign. Consequently, if we denote by $\pi_{U^{*}}$ the Poisson structure on $U^{*}$ such that $\left(U^{*}, \pi_{U^{*}}\right)$ is the dual Poisson Lie group of $\left(U, \pi_{U}\right)$, then every $U^{*}$-orbit in $\mathcal{L}(\mathfrak{d})$ is a $\left(U^{*},-\pi_{U^{*}}\right)$-homogeneous Poisson space.

We now look at the Drinfeld map P $: U \cdot \mathfrak{l} \rightarrow \mathcal{L}(\mathfrak{d})$ for the $\left(U, \pi_{U}\right)$-homogeneous Poisson space $(U \cdot \mathfrak{l}, \Pi)$ (see Definition 2.4).

THEOREM 2.21. - For any $\mathfrak{l} \in \mathcal{L}(\mathfrak{d})$, the Lagrangian subalgebra of $\mathfrak{d}$ associated to $(U \cdot \mathfrak{l}, \Pi)$ at $\mathfrak{l}$ is

$$
\mathrm{T}(\mathfrak{l})=\mathfrak{u}_{\mathfrak{l}}+\left(\mathfrak{u}+\mathfrak{u}_{\mathfrak{l}}^{\perp}\right) \cap \mathfrak{l},
$$

where $\mathfrak{u}_{\mathfrak{l}}$ is the normalizer subalgebra of $\mathfrak{l}$ in $\mathfrak{u}$, and $\mathfrak{u}_{\mathfrak{l}}^{\perp}=\left\{\xi \in \mathfrak{u}^{*}:\left.\xi\right|_{\mathfrak{u}_{\mathfrak{l}}}=0\right\}$.

Proof. - Denote by $\mathfrak{l}^{\prime}$ the Lagrangian subalgebra associated to $(U \cdot \mathfrak{l}, \Pi)$ at $\mathfrak{l}$. We need to show that $\mathfrak{l}^{\prime}=\mathrm{T}(\mathfrak{l})$. By definition,

$$
\left.\mathfrak{l}^{\prime}=\left\{x+\xi: x \in \mathfrak{u}, \xi \in \mathfrak{u}_{\mathfrak{l}}^{\perp}, \xi\right\lrcorner \Pi(\mathfrak{l})=x+\mathfrak{u}_{\mathfrak{l}}\right\} .
$$


Let $\xi \in \mathfrak{u}_{\mathfrak{l}}^{\perp}$. Since the inclusion

$$
(U \cdot \mathfrak{l}, \Pi) \longrightarrow(D \cdot \mathfrak{l}, \Pi)
$$

is a Poisson map, it suffices to compute $\left.\left(\left(\kappa^{*}\right)^{-1}(\xi+x)\right)\right\lrcorner \Pi(\mathfrak{l})$ for any $x \in \mathfrak{u}$ such that $\xi+x \in \mathfrak{d}_{\mathfrak{l}}^{0}$, where $\Pi(\mathfrak{l})$ is regarded as a bi-vector at $\mathfrak{l} \in D \cdot \mathfrak{l}$, and $\left(\kappa^{*}\right)^{-1}: \mathrm{T}_{\mathfrak{l}}^{*}(D \cdot \mathfrak{l}) \rightarrow \mathfrak{d}_{\mathfrak{l}}^{\circ}$ is the isomorphism induced by $\kappa: \mathfrak{d} \rightarrow \mathrm{T}_{\mathfrak{l}}(D \cdot \mathfrak{l})$. In the proof of Theorem 2.18, we showed that $\left.\left(\kappa^{*}\right)^{-1}(\xi+x)\right\lrcorner \Pi(\mathfrak{l})=\kappa(x)$. As a result, we see that

$$
\begin{aligned}
\mathfrak{l}^{\prime} & =\left\{x+\xi: x \in \mathfrak{u}, \xi \in \mathfrak{u}_{\mathfrak{l}}^{\perp}, \xi+x_{1} \in \mathfrak{d}_{\mathfrak{l}}^{\circ} \text { for some } x_{1}=x \bmod \left(\mathfrak{u}_{\mathfrak{l}}\right)\right\} \\
& =\mathfrak{u}_{\mathfrak{l}}+\#\left(\mathfrak{d}_{\mathfrak{l}}^{\circ}\right) .
\end{aligned}
$$

Now, the inclusions $\mathfrak{u}_{\mathfrak{l}} \subset \mathfrak{d}_{\mathfrak{l}}$ and $\mathfrak{l} \subset \mathfrak{d}_{\mathfrak{l}}$ induce inclusions $\#\left(\mathfrak{d}_{\mathfrak{l}}^{\circ}\right) \subset \mathfrak{u}+\mathfrak{u}_{\mathfrak{l}}^{\perp}$ and $\#\left(\mathfrak{d}_{\mathfrak{l}}^{\circ}\right) \subset \mathfrak{l}$, so $\#\left(\mathfrak{d}_{\mathfrak{l}}^{\circ}\right) \subset\left(\mathfrak{u}+\mathfrak{u}_{\mathfrak{l}}^{\perp}\right) \cap \mathfrak{l}$. Hence,

$$
\mathfrak{u}_{\mathfrak{l}}+\#\left(\mathfrak{d}_{\mathfrak{l}}^{\circ}\right) \subset \mathfrak{u}_{\mathfrak{l}}+\left(\mathfrak{u}+\mathfrak{u}_{\mathfrak{l}}^{\perp}\right) \cap \mathfrak{l}=\mathrm{T}(\mathfrak{l}) .
$$

On the other hand, it is obvious that $\mathrm{T}(\mathfrak{l})$ is isotropic, so its dimension is at most $n$. Since $\mathfrak{l}^{\prime}$ has dimension $n$, we must have $\mathfrak{l}^{\prime}=\mathrm{T}(\mathfrak{l})$.

Remark 2.22. - The map $T: \mathcal{L}(\mathfrak{d}) \rightarrow \mathcal{L}(\mathfrak{d})$ is not continuous in general. For example, consider the Lie bialgebra in Example 2.1 for $\mathfrak{g}=\mathfrak{s l}(3, \mathbb{C})$. Choose $H \in \mathfrak{a}$ with the property that both simple roots are positive on $H$. It is possible to choose a real form $\mathfrak{g}_{0}$ of $\mathfrak{s l}(3, \mathbb{C})$ that is isomorphic to $\mathfrak{s l}(3, \mathbb{R})$ such that the curve $\gamma_{t}=\exp \left(\operatorname{ad}_{t H}\right)(\mathfrak{s l}(3, \mathbb{R}))$ in $\mathcal{L}=\mathcal{L}(\mathfrak{g})$ has a limit $\gamma_{\infty}=\mathfrak{h}^{\tau}+\mathfrak{n}$, where $\mathfrak{h}=\mathfrak{a}+\mathfrak{t}$ is a Cartan subalgebra of $\mathfrak{s l}(3, \mathbb{C})$, and $\tau$ is a conjugate linear automorphism such that $\operatorname{dim}\left(\mathfrak{h}^{\tau} \cap \mathfrak{t}\right)=1$. Since the normalizer of $\mathfrak{g}_{0}$ in $\mathfrak{s l}(3, \mathbb{C})$ is $\mathfrak{g}_{0}$, the normalizer of $\gamma_{t}$ in $\mathfrak{s l}(3, \mathbb{C})$ is $\gamma_{t}$ for $t \neq \infty$. Consequently, $\mathrm{T}\left(\gamma_{t}\right)=\gamma_{t}$ for all $t<\infty$. On the other hand, it is easy to check that $\mathrm{T}\left(\gamma_{\infty}\right)=\mathfrak{t}+\mathfrak{n}$. It follows that $T$ is not continuous. This example can be generalized to any real form corresponding to a nontrivial diagram automorphism (see Remark 4.9 for a generalization of this example).

Assume now that $(M, \pi)$ is an arbitrary $\left(U, \pi_{U}\right)$-homogeneous Poisson space. Consider the Drinfeld map

$$
\mathrm{P}: M \longrightarrow \mathcal{L}(\mathfrak{d}): m \longmapsto \mathfrak{l}_{m} .
$$

By Theorem 2.3, $\mathrm{P}$ is a submersion of $M$ onto the $U$-orbit $\mathcal{O}=U \cdot \mathfrak{l}_{m}$ in $\mathcal{L}(\mathfrak{d})$ for any $m \in M$.

THEOREM 2.23. - The Drinfeld map

$$
\mathrm{P}:(M, \pi) \longrightarrow(\mathcal{O}, \Pi)
$$

is a Poisson map.

Proof. - Fix $m \in M$. Let $\mathfrak{l}=\mathfrak{l}_{m}$. Then $\mathcal{O}=U \cdot \mathfrak{l}$. Since both $(M, \pi)$ and $(\mathcal{O}, \Pi)$ are $\left(U, \pi_{U}\right)$ homogeneous, it is enough to show that

$$
\mathrm{P}_{*} \pi(m)=\Pi(\mathfrak{l}) .
$$

Let $U_{m}$ and $U_{\mathfrak{l}}$ be respectively the stabilizer subgroup of $U$ at $m$ and the normalizer subgroup of $\mathfrak{l}$ in $U$. Their Lie algebras are respectively $\mathfrak{l} \cap \mathfrak{u}$ and $\mathfrak{u}_{\mathfrak{l}}$. Since $\mathrm{P}$ is $U$-equivariant, we have $U_{m} \subset U_{\text {l. Identify }}$

$$
M \cong U / U_{m}, \quad \mathcal{O} \cong U / U_{\mathfrak{l}}
$$


Then the map P becomes

$$
\mathrm{P}: U / U_{m} \longrightarrow U / U_{\mathfrak{l}}: u U_{m} \longmapsto u U_{\mathfrak{l}}
$$

and we have

$$
\pi(m) \in \wedge^{2}(\mathfrak{u} /(\mathfrak{l} \cap \mathfrak{u})), \quad \Pi(\mathfrak{l}) \in \wedge^{2}\left(\mathfrak{u} / \mathfrak{u}_{\mathfrak{l}}\right) .
$$

Thus we only need to show that $\pi(m)$ goes to $\Pi(\mathfrak{l})$ under the map

$$
j: \mathfrak{u} /(\mathfrak{l} \cap \mathfrak{u}) \longrightarrow \mathfrak{u} / \mathfrak{u}_{\mathfrak{l}}: x+\mathfrak{l} \cap \mathfrak{u} \longmapsto x+\mathfrak{u}_{\mathfrak{l}} .
$$

But this follows from a general linear algebra fact which we state as a lemma below.

LEMMA 2.24. - Let $V$ be an $n$-dimensional vector space and let $V^{*}$ be its dual space. On the direct sum vector space $V \oplus V^{*}$, consider the symmetric product $\langle$,$\rangle defined by$

$$
\langle x+\xi, y+\eta\rangle=(x, \eta)+(y, \xi), \quad x, y \in V, \xi, \eta \in V^{*} .
$$

1) Let $V_{0}$ be any subspace of $V$. For $\lambda \in \wedge^{2}\left(V / V_{0}\right)$, define

$$
\left.W_{\lambda}=\left\{x+\xi: x \in V, \xi \in V^{*},\left.\xi\right|_{V_{0}}=0, \xi\right\lrcorner \lambda=x+V_{0}\right\} .
$$

Then $\lambda \mapsto W_{\lambda}$ is a one-to-one correspondence between elements in $\wedge^{2}\left(V / V_{0}\right)$ and maximal isotropic subspaces $W$ of $V \oplus V^{*}$ such that $W \cap V=V_{0}$.

2) Let $V_{1}$ be another subspace of $V$ such that $V_{0} \subset V_{1}$. Let

$$
j: V / V_{0} \longrightarrow V / V_{1}: v+V_{0} \longmapsto v+V_{1}
$$

be the natural projection. Let $\lambda_{0} \in \wedge^{2}\left(V / V_{0}\right)$ and $\lambda_{1} \in \wedge^{2}\left(V / V_{1}\right)$. Then $j\left(\lambda_{0}\right)=\lambda_{1}$ if and only if

$$
W_{\lambda_{1}}=V_{1}+\left(V \oplus V_{1}^{\perp}\right) \cap W_{\lambda_{0}},
$$

where $V_{1}^{\perp}=\left\{\xi \in V^{*}:\left.\xi\right|_{V_{1}}=0\right\}$.

Proof. - 1) Given $\lambda \in \wedge^{2}\left(V / V_{0}\right)$, it is easy to see that $W_{\lambda}$ is maximal isotropic with respect to $\langle$,$\rangle and that W_{\lambda} \cap V=V_{0}$. Conversely, if $W$ is a maximal isotropic subspace of $V \oplus V^{*}$ such that $W \cap V=V_{0}$, then

$$
\left\{\xi \in V^{*}: x+\xi \in W \text { for some } x \in V\right\}=V_{0}^{\perp}=\left\{\xi \in V^{*}:\left.\xi\right|_{V_{0}}=0\right\} .
$$

Define

$$
f:\left(V / V_{0}\right)^{*} \longrightarrow V / V_{0}: \xi \longmapsto x+V_{0},
$$

where $\xi \in\left(V / V_{0}\right)^{*} \cong V_{0}^{\perp}$ and $x \in V$ is such that $x+\xi \in W$. Then $f$ is well defined and is skew-symmetric. Thus there exists $\lambda \in \wedge^{2}\left(V / V_{0}\right)$ such that $\left.f(\xi)=\xi\right\lrcorner \lambda$ for all $\xi \in\left(V / V_{0}\right)^{*}$. It is then easy to check that $W=W_{\lambda}$.

2) One way to prove this fact is to take a basis for $V_{0}$, extend it first to a basis for $V_{1}$ and then extend it further to a basis of $V$. One can then write down all the spaces in (8) using these basis vectors and compare them. We omit the details.

As a special case of Theorem 2.23, we have: 
Corollary 2.25. - For any $\mathfrak{l} \in \mathcal{L}(\mathfrak{d})$ with Property $\mathrm{C}$ and any $\left(U, \pi_{U}\right)$-homogeneous space $\left(U / U_{1}, \pi\right)$ determined by $\mathfrak{l}$ (see Definitions 2.7 and 2.9 ), the map

$$
P:\left(U / U_{1}, \pi\right) \longrightarrow(U \cdot \mathfrak{l}, \Pi): u U_{1} \longmapsto \operatorname{Ad}_{u} \mathfrak{l}
$$

is Poisson.

\subsection{Model points in $\mathcal{L}(\mathfrak{d})$}

DEFINITION 2.26. - We say that a Lagrangian subalgebra $\mathfrak{l}$ is a $\mathfrak{u}$-model point (in $\mathcal{L}(\mathfrak{d})$ ) if $\mathfrak{l} \cap \mathfrak{u}=\mathfrak{u}_{\mathfrak{l}}$, where $\mathfrak{u}_{\mathfrak{l}}$ is the normalizer subalgebra of $\mathfrak{l}$ in $\mathfrak{u}$.

It is easy to see that the set of model points in $\mathcal{L}(\mathfrak{d})$ is invariant under the $U$-action. Observe also that $\mathfrak{l}$ is a model point if and only if $\mathrm{T}(\mathfrak{l})=\mathfrak{l}$. In general, $T^{2} \neq T$. See Remark 3.6 for an example. For the rest of the section, we refer to $\mathfrak{u}$-model points simply as model points.

Every model point has Property $\mathrm{C}$, for if $\mathfrak{l} \in \mathcal{L}(\mathfrak{d})$ is a model point, the connected subgroup $U_{\mathfrak{l}}^{\prime}$ of $U$ with Lie algebra $\mathfrak{l} \cap \mathfrak{u}$ is the identity component of the stabilizer subgroup $U_{\mathfrak{l}}$ of $\mathfrak{l}$ in $U$, so $U_{\mathfrak{l}}^{\prime}$ is closed. Consequently, $\mathfrak{l}$ determines a $\left(U, \pi_{U}\right)$-homogeneous Poisson structure on any $U / U_{1}$, where $U_{1}$ is a closed subgroup of $U_{\mathfrak{l}}$, and has the same Lie algebra $\mathfrak{l} \cap \mathfrak{u}=\mathfrak{u}_{\mathfrak{l}}$ (see Corollary 2.8 and Definition 2.9). In this case, the map $P$ in (9) is a local diffeomorphism (in addition to being a Poisson map), and is thus a covering map. Therefore, the orbit $U \cdot \mathfrak{l}$, together with the Poisson structure $\Pi$, is a model (up to local diffeomorphism) of any $\left(U, \pi_{U}\right)$-homogeneous Poisson space $\left(U / U_{1}, \pi\right)$ determined by $\mathfrak{l}$. This is the reason we call $\mathfrak{l}$ a model point in $\mathcal{L}(\mathfrak{d})$. Moreover, for a model point $\mathfrak{l}$, the Drinfeld map for the Poisson homogeneous space $(U \cdot \mathfrak{l}, \Pi)$ is the inclusion $\operatorname{map} U \cdot \mathfrak{l} \subset \mathcal{L}(\mathfrak{d})$.

Example 2.27. - Consider the Lie bialgebra $(\mathfrak{k}, \mathfrak{a}+\mathfrak{n})$ in Example 2.1. The Lagrangian subalgebra $\mathfrak{l}=\mathfrak{a}+\mathfrak{n}$ is not a model point because $\mathfrak{l} \cap \mathfrak{k}=0$ while the normalizer subalgebra of $\mathfrak{l}$ in $\mathfrak{k}$ is $\mathfrak{t}=i \mathfrak{a}$. However, $T(\mathfrak{l})=\mathfrak{t}+\mathfrak{n}$ is a model point, as is any real form of $\mathfrak{g}$. In this case, we will show that every point in a certain irreducible component $\mathcal{L}(\mathfrak{d})_{0}$ of $\mathcal{L}(\mathfrak{d})$ is a model point.

When $\mathfrak{l}$ is a model point and when its normalizer subgroup $U_{\mathfrak{l}}$ in $U$ is not connected, the $\left(U, \pi_{U}\right)$-homogeneous Poisson spaces $\left(U / U_{1}, \pi\right)$ determined by $\mathfrak{l}$ might have non-trivial symmetries, as is shown in the following proposition.

Proposition 2.28. - Let l be a model point and let $\left(U / U_{1}, \pi\right)$ be any $\left(U, \pi_{U}\right)$-homogeneous Poisson space determined by $l$. Then all covering transformations for the covering map

$$
P:\left(U / U_{1}, \pi\right) \longrightarrow\left(U / U_{\mathfrak{l}}, \Pi\right): u U_{1} \longmapsto u U_{\mathfrak{l}}
$$

are Poisson isometries for $\left(U / U_{1}, \pi\right)$.

Proof. - Let $f: U / U_{1} \rightarrow U / U_{1}$ be a covering transformation, so $P \circ f=P$. We know that $f$ is smooth because it must be of the form

$$
f\left(u U_{1}\right)=u u_{0} U_{1}
$$

for some $u_{0}$ in the normalizer subgroup of $U_{1}$ in $U_{\text {r. Let }} x \in U / U_{1}$ be arbitrary. We need to show that $f_{*} \pi(x)=\pi(f(x))$. Since $P$ is a local diffeomorphism, it is enough to show that $f_{*} \pi(x)$ and $\pi(f(x))$ have the same image under $P$. Now, since $P$ is a Poisson map and since $P \circ f=P$, we have 


$$
\begin{aligned}
P_{*} f_{*} \pi(x) & =(P \circ f)_{*} \pi(x)=P_{*} \pi(x)=\Pi(P(x)), \\
P_{*} \pi(f(x)) & =\Pi(P(f(x)))=\Pi(P(x)) .
\end{aligned}
$$

Thus $P_{*} f_{*} \pi(x)=P_{*} \pi(f(x))$, and $f$ is a Poisson map.

In particular, in the case when $U_{1}=U_{\mathfrak{l}}^{\prime}$ is the identity connected component of $U_{\mathfrak{l}}$, the group $U_{\mathfrak{l}} / U_{\mathfrak{l}}^{\prime}$ acts on $U / U_{\mathfrak{l}}^{\prime}$ as symmetries for $\left(U, \pi_{U}\right)$-homogeneous Poisson structure on $U / U_{\mathfrak{l}}^{\prime}$ determined by $\mathfrak{l}$.

\section{Lagrangian subalgebras of $\mathfrak{g}$}

In the remainder of the paper, we will concentrate on the Lie bialgebra $(\mathfrak{k}, \mathfrak{a}+\mathfrak{n})$ as described in Example 2.1. We first fix more notation.

Throughout the rest of the paper, $\mathfrak{k}$ will be a compact semi-simple Lie algebra and $\mathfrak{g}=\mathfrak{k}_{\mathbb{C}}$ its complexification. The Killing form of $\mathfrak{g}$ will be denoted by $\langle\langle\rangle$,$\rangle . Let G$ be the adjoint group of $\mathfrak{g}$, let $K$ be the connected subgroup of $G$ with Lie algebra $\mathfrak{k}$, let $T \subset K$ be a maximal torus with Lie algebra $\mathfrak{t}$. Let $\mathfrak{h}=\mathfrak{t}_{\mathbb{C}} \subset \mathfrak{g}$ be the complexification of $\mathfrak{t}$. Let $\Sigma$ be the set of roots of $\mathfrak{g}$ with respect to $\mathfrak{h}$ with the root decomposition

$$
\mathfrak{g}=\mathfrak{h}+\sum_{\alpha \in \Sigma} \mathfrak{g}_{\alpha}
$$

Let $\Sigma_{+}$be a choice of positive roots, and let $S\left(\Sigma_{+}\right)$be the set of simple roots in $\Sigma_{+}$. We will also say $\alpha>0$ for $\alpha \in \Sigma_{+}$. Set $\mathfrak{a}=i \mathfrak{t}$ and let $\mathfrak{n}$ be the complex subspace spanned by all the positive root vectors. Then we can identify $\mathfrak{k}^{*}$ with $\mathfrak{a}+\mathfrak{n}$ (here $\mathfrak{n}$ is regarded as a real Lie subalgebra of $\mathfrak{g})$ through the pairing defined by twice the imaginary part of the Killing form $\langle\langle$,$\rangle . This way,$ $(\mathfrak{k}, \mathfrak{a}+\mathfrak{n})$ becomes a Lie bialgebra whose double is $\mathfrak{g}=\mathfrak{k}+\mathfrak{a}+\mathfrak{n}$ (Iwasawa decomposition of $\mathfrak{g}$ ). Let $\pi_{K}$ be the Poisson structure on $K$ such that $\left(K, \pi_{K}\right)$ is a Poisson Lie group with tangent Lie bialgebra $(\mathfrak{k}, \mathfrak{a}+\mathfrak{n})$. We can describe $\pi_{K}$ explicitly as follows: Let $\theta$ be the complex conjugation of $\mathfrak{g}$ defined by $\mathfrak{k}$. Let $\langle\langle,\rangle\rangle_{\theta}$ be the Hermitian positive definite inner product on $\mathfrak{g}$ given by

$$
\langle\langle x, y\rangle\rangle_{\theta}=-\langle\langle x, \theta y\rangle\rangle, \quad x, y \in \mathfrak{g} .
$$

For each $\alpha \in \Sigma_{+}$, choose $E_{\alpha} \in \mathfrak{g}_{\alpha}$ such that $\left\langle\left\langle E_{\alpha}, E_{\alpha}\right\rangle_{\theta}=1\right.$. Let $E_{-\alpha}=-\theta\left(E_{\alpha}\right) \in \mathfrak{g}_{-\alpha}$ so that $\left\langle\left\langle E_{\alpha}, E_{-\alpha}\right\rangle\right\rangle=1$. Set

$$
X_{\alpha}=E_{\alpha}-E_{-\alpha}=E_{\alpha}+\theta\left(E_{\alpha}\right), \quad Y_{\alpha}=i\left(E_{\alpha}+E_{-\alpha}\right)=i E_{\alpha}+\theta\left(i E_{\alpha}\right) .
$$

Then

$$
\mathfrak{k}=\mathfrak{t}+\operatorname{span}_{\mathbb{R}}\left\{X_{\alpha}, Y_{\alpha}: \alpha \in \Sigma_{+}\right\} .
$$

The Poisson bivector field on $K$ is given by

$$
\pi_{K}(k)=r_{k} \Lambda-l_{k} \Lambda, \quad k \in K
$$

where

$$
\Lambda=\frac{1}{4} \sum_{\alpha \in \Sigma_{+}} X_{\alpha} \wedge Y_{\alpha} \in \mathfrak{k} \wedge \mathfrak{k} .
$$


Recall that a real subalgebra $\mathfrak{l}$ of $\mathfrak{g}$ is Lagrangian if $\operatorname{Im}\langle\langle x, y\rangle\rangle=0$ for all $x, y \in \mathfrak{l}$ and if $\operatorname{dim}_{\mathbb{R}} \mathfrak{l}=\operatorname{dim}_{\mathbb{C}} \mathfrak{g}$. These Lagrangian subalgebras give rise to $\left(K, \pi_{K}\right)$ Poisson-homogeneous spaces by Drinfeld's theorem. The set of all Lagrangian subalgebras of $\mathfrak{g}$ will be denoted by $\mathcal{L}$. It is an algebraic subset of the Grassmannian $\operatorname{Gr}(n, \mathfrak{g})$ of $n$-dimensional subspaces of $\mathfrak{g}$ (regarded as a $2 n$-dimensional real vector space).

In this section, we will decompose $\mathcal{L}$ into a finite union of manifolds.

\subsection{Karolinsky's classification}

E. Karolinsky [12] has determined all Lagrangian subalgebras $\mathfrak{l}$ of $\mathfrak{g}$. To describe his result, we need some notation. Let $S \subset S\left(\Sigma_{+}\right)$be a subset of the set of simple roots, and let $[S]$ be the set of roots in the linear span of $S$. Consider

$$
\mathfrak{m}_{S}=\mathfrak{h} \oplus\left(\bigoplus_{\alpha \in[S]} \mathfrak{g}_{\alpha}\right), \quad \mathfrak{n}_{S}=\bigoplus_{\alpha \in \Sigma_{+}-[S]} \mathfrak{g}_{\alpha}, \quad \mathfrak{p}_{S}=\mathfrak{m}_{S}+\mathfrak{n}_{S},
$$

so that $\mathfrak{p}_{S}$ is a parabolic subalgebra of type $S, \mathfrak{n}_{S}$ is its nilradical, and $\mathfrak{m}_{S}$ is a Levi factor. Let $\mathfrak{m}_{S, 1}=\left[\mathfrak{m}_{S}, \mathfrak{m}_{S}\right]$ be the (semi-simple) derived algebra of $\mathfrak{m}_{S}$. The center of $\mathfrak{m}_{S}$ is

$$
\mathfrak{z}_{S}=\left\{H \in \mathfrak{h}: \alpha_{i}(H)=0, \forall \alpha_{i} \in S\right\},
$$

which is also the orthogonal complement of $\mathfrak{m}_{S, 1}$ in $\mathfrak{m}_{S}$ with respect to the Killing form of $\mathfrak{g}$ restricted to $\mathfrak{m}_{S}$. Thus the restriction of the Killing form to $\mathfrak{z}_{S}$ is nondegenerate, and we may consider Lagrangian subspaces of $\mathfrak{z}_{S}$ (regarded as a real vector space) with respect to the restriction to $\mathfrak{z}_{S}$ of the imaginary part of the Killing form.

Now for any subset $S$ of the set of simple roots, a Lagrangian subspace $V$ of $\mathfrak{z}_{S}$, and a real form $\mathfrak{m}_{S, 1}^{\tau}$ of $\mathfrak{m}_{S, 1}$ such that the Killing form of $\mathfrak{g}$ is real on $\mathfrak{m}_{S, 1}^{\tau}$, set

$$
\mathfrak{l}(S, V, \tau)=\mathfrak{m}_{S, 1}^{\tau} \oplus V \oplus \mathfrak{n}_{S}
$$

It is easy to see that it is a Lagrangian subalgebra of $\mathfrak{g}$.

DEFINITION 3.1. - We will call $\mathfrak{l}(S, V, \tau)$ the standard Lagrangian subalgebra associated to $(S, V, \tau)$.

THEOREM 3.2 ([12]). - Every Lagrangian subalgebra of $\mathfrak{g}$ is of the form $\operatorname{Ad}_{k}(\mathfrak{l}(S, V, \tau))$ for some $k \in K$.

Note that the nilradical of $\operatorname{Ad}_{k}(\mathfrak{l}(S, V, \tau))$ is $\operatorname{Ad}_{k}\left(\mathfrak{n}_{S}\right)$. Denote by $P_{S}$ the connected subgroup of the adjoint group $G$ of $\mathfrak{g}$ with Lie algebra $\mathfrak{p}_{S}$.

Proposition 3.3. - Let

$$
\mathfrak{l}=\operatorname{Ad}_{k}(\mathfrak{l}(S, V, \tau))=\operatorname{Ad}_{k_{1}}\left(\mathfrak{l}\left(S_{1}, V_{1}, \tau_{1}\right)\right)
$$

be a Lagrangian subalgebra. Then $S=S_{1}, V=V_{1}, k^{-1} k_{1} \in P_{S}$, and $\tau$ is conjugate to $\tau_{1}$ in $K \cap P_{S}$.

Proof. - We have $\operatorname{Ad}_{k^{-1} k_{1}}\left(\mathfrak{l}\left(S_{1}, V_{1}, \tau_{1}\right)\right)=\mathfrak{l}(S, V, \tau)$. Using the fact that conjugate algebras have conjugate nilradicals, it follows easily that $\operatorname{Ad}_{k^{-1} k_{1}} \mathfrak{n}_{S_{1}}=\mathfrak{n}_{S}$. From the definition of $\mathfrak{n}_{S}$, it follows that $S=S_{1}$. Since a parabolic subgroup is the normalizer of its nilradical, $k^{-1} k_{1} \in P_{S}$. The remaining claims follow from the facts that $\mathfrak{n}_{S}$ is an ideal and $\mathfrak{z} S$ is central in $\mathfrak{m}_{S}$. 


\section{2. $\mathfrak{k}$-model points in $\mathcal{L}$}

Recall that a Lagrangian subalgebra $\mathfrak{l}$ of $\mathfrak{g}$ is a $\mathfrak{k}$-model point if $\mathfrak{k} \cap \mathfrak{l}=\mathfrak{k}_{\mathfrak{l}}$, where $\mathfrak{k}_{\mathfrak{l}}$ is the normalizer subalgebra of $\mathfrak{l}$ in $\mathfrak{k}$. In this section, we refer to $\mathfrak{k}$-model points simply as model points.

LEMmA 3.4. - The normalizer of $\operatorname{Ad}_{k}\left(\mathfrak{l}(S, V, \tau)\right.$ in $\mathfrak{g}$ is $\operatorname{Ad}_{k} \mathfrak{r}(S, \tau)$, where

$$
\mathfrak{r}(S, \tau)=\mathfrak{m}_{S, 1}^{\tau} \oplus \mathfrak{z} S \oplus \mathfrak{n}_{S} .
$$

Proof. - It suffices to prove the statement when $k=e$, the identity element of $K$. It is clear that $\mathfrak{r}(S, \tau)$ normalizes $\mathfrak{l}(S, V, \tau)$. Conversely, if $X \in \mathfrak{g}$ normalizes $\mathfrak{l}(S, V, \tau)$, it normalizes its nilradical $\mathfrak{n}_{S}$, so it normalizes the perpendicular $\mathfrak{p}_{S}$ of $\mathfrak{n}_{S}$. Since $\mathfrak{p}_{S}$ is parabolic, it equals its own normalizer, so $X \in \mathfrak{p}_{S}$. Write $X=X_{1}+X_{2}$, with $X_{1} \in \mathfrak{m}_{S}$ and $X_{2} \in \mathfrak{n}_{S}$. Then $X_{1}$ normalizes $\mathfrak{m}_{S, 1}^{\tau}$. Since a real form of a complex semi-simple Lie algebra $\mathfrak{m}$ is its own normalizer in $\mathfrak{m}$ (see Lemma 3.10), we know that $X_{1} \in \mathfrak{m}_{S, 1}^{\tau}+\mathfrak{z}_{S}$.

Proposition 3.5. - The Lagrangian subalgebra $\operatorname{Ad}_{k}(\mathfrak{l}(S, V, \tau))$ is a model point if and only if $V=\mathfrak{z} S \cap \mathfrak{t}$.

Proof. - Since the set of model points is $K$-invariant, it suffices to prove the proposition when $k=e$. Let $\mathfrak{k}_{\mathfrak{l}}=\mathfrak{r}(S, \tau) \cap \mathfrak{k}$ be the normalizer of $\mathfrak{l}(S, V, \tau)$ in $\mathfrak{k}$, where $\mathfrak{r}(S, \tau)=\mathfrak{m}_{S, 1}^{\tau} \oplus \mathfrak{z} S \oplus \mathfrak{n}_{S}$ as in Lemma 3.4. Then

$$
\mathfrak{k}_{\mathfrak{l}} / \mathfrak{k} \cap \mathfrak{l}(S, V, \tau)=(\mathfrak{z} S \cap \mathfrak{t}) /(V \cap \mathfrak{t}),
$$

because $\mathfrak{z}_{S} \cap \mathfrak{k}=\mathfrak{z}_{S} \cap \mathfrak{t}$. The proposition now follows from the definition of model points.

Remark 3.6. - In fact, essentially the same argument shows that if $\mathfrak{l}=\operatorname{Ad}_{k}(\mathfrak{l}(S, V, \tau))$, then $\mathrm{T}(\mathfrak{l})=\operatorname{Ad}_{k}(\mathfrak{l}(S, \mathfrak{z} S \cap \mathfrak{t}, \tau)$ ) (see Theorem 2.21 for the definition of $\mathrm{T}(\mathfrak{l})$ ). It follows that $\mathrm{T}(\mathrm{T}(\mathfrak{l}))=\mathrm{T}(\mathfrak{l})$ for $\mathfrak{l} \in \mathcal{L}$. For a general Lie bialgebra, $T \circ T \neq T$. Indeed, for a Lie algebra $\mathfrak{u}$, we can form a Lie bialgebra $\left(\mathfrak{u}, \mathfrak{u}^{*}\right)$, where $\mathfrak{u}^{*}$ has the abelian Lie algebra structure. Its double is the semi-direct product Lie algebra structure on $\mathfrak{u}+\mathfrak{u}^{*}$ defined by the co-adjoint action of $\mathfrak{u}$ on $\mathfrak{u}^{*}$. Consider the case when $\mathfrak{u}$ is the three dimensional Heisenberg algebra with basis $\{X, Y, Z\}$ with $Z$ central and $[X, Y]=Z$, and let $f_{X}, f_{Y}, f_{Z}$ be the dual basis. Let $\mathfrak{l}$ be the Lagrangian subalgebra spanned by $X, f_{Y}$ and $f_{Z}$. Then $\mathrm{T}(\mathfrak{l})$ is spanned by $X, Z$ and $f_{Y}$ while $\mathrm{T}(\mathrm{T}(\mathfrak{l}))=\mathfrak{u}$.

\section{COROLLARY 3.7. - The adjoint group $G$ of $\mathfrak{g}$ preserves the set of model points.}

Proof. - It suffices to consider model points $\mathfrak{l}\left(S, \mathfrak{z}_{S} \cap \mathfrak{t}, \tau\right)$. Let $P_{S}, M_{S}$ and $N_{S}$ be the connected Lie groups with Lie algebra $\mathfrak{p}_{S}, \mathfrak{m}_{S}$ and $\mathfrak{n}_{S}$ respectively. Since $K$ acts transitively on $G / P_{S}$ and preserves the set of model points, it suffices to prove that $\operatorname{Ad}_{p}\left(\mathfrak{l}\left(S, \mathfrak{z}_{S} \cap \mathfrak{t}, \tau\right)\right)$ is a model point for $p \in P_{S}$. Using the Levi decomposition $P_{S}=M_{S} N_{S}$ we write $p=m n$, where $m \in M_{S}$ and $n \in N_{S}$. Since $\operatorname{Ad}_{n} \mathfrak{l}\left(S, \mathfrak{z}_{S} \cap \mathfrak{t}, \tau\right)=\mathfrak{l}\left(S, \mathfrak{z}_{S} \cap \mathfrak{t}, \tau\right)$, it suffices to prove that $\operatorname{Ad}_{m}$ preserves model points in $\mathfrak{p}_{S}$, which follows because $M$ acts trivially on $\mathfrak{z}_{S}$.

Remark 3.8. - In general, the adjoint group of the double Lie algebra does not preserve the set of model points. Indeed, let $\mathfrak{g}$ be a semi-simple Lie algebra with triangular decomposition $\mathfrak{g}=\mathfrak{n}+\mathfrak{h}+\mathfrak{n}_{-}$, Borel subalgebra $\mathfrak{b}_{+}=\mathfrak{h}+\mathfrak{n}$ and opposite Borel $\mathfrak{b}_{-}=\mathfrak{h}+\mathfrak{n}_{-}$. Then the Lie algebra $\mathfrak{d}=\mathfrak{g} \oplus \mathfrak{h}$ is the double of the pair $\left(\mathfrak{b}_{+}, \mathfrak{b}_{-}\right)$with embeddings $i_{ \pm}: \mathfrak{b}_{ \pm} \rightarrow \mathfrak{d}$ given by $i_{ \pm}(H+x)=(H+x, \pm H)$ with $H \in \mathfrak{h}, x \in \mathfrak{n}$ or $\mathfrak{n}_{-}$. Let $n \in N_{G}(\mathfrak{t})$ be a representative for the long element of the Weyl group. Then although $\mathfrak{b}_{+}$is clearly a $\mathfrak{b}_{+}$-model point, $\operatorname{Ad}_{n}\left(\mathfrak{b}_{+}\right)$is not a $\mathfrak{b}_{+}$-model point. 
In the following Sections 3.3, 3.4, and 3.5, we study separately the pieces that come into the Karolinsky classification, and we write the variety $\mathcal{L}$ of all Lagrangian subalgebras of $\mathfrak{g}$ as a finite disjoint union of smooth submanifolds of the Grassmannian $\operatorname{Gr}(n, \mathfrak{g})$.

\subsection{Lagrangian subspaces of $\mathfrak{z}_{S}$}

For a subset $S$ of the set of simple roots, let $\mathfrak{z}_{S}$ be given as in (11). Since the Killing form of $\mathfrak{g}$ is nondegenerate on $\mathfrak{z}_{S}$, its imaginary part $B$ is a nondegenerate symmetric bilinear form of index $(z, z)$ on $\mathfrak{z}_{S}$, now regarded as a $2 z$-dimensional real vector space. Denote by $\mathcal{L}_{\mathfrak{z}_{S}}$ the variety of Lagrangian subspaces of $\mathfrak{z}_{S}$ with respect to $B$, and denote by $\operatorname{Gr}\left(z, \mathfrak{z}_{S}\right)$ the Grassmannian of $z$-dimensional real subspaces of $\mathfrak{z}_{S}$, so $\mathcal{L}_{\mathfrak{z}_{S}} \subset \operatorname{Gr}\left(z, \mathfrak{z}_{S}\right)$.

PROPOSITION 3.9. - $\mathcal{L}_{\mathfrak{z}_{S}}$ is a smooth algebraic subvariety of $\operatorname{Gr}\left(z, \mathfrak{z}_{S}\right)$ and is diffeomorphic to the real orthogonal group $\mathrm{O}(z)$. We denote the two connected components of $\mathcal{L}_{\mathfrak{z} S}$ by $\mathcal{L}_{\mathfrak{z}_{S, \varepsilon}}$, for $\varepsilon= \pm 1$, with $\mathcal{L}_{\mathfrak{z}_{S, 1}}$ being the component containing $\mathfrak{z}_{S} \cap \mathfrak{t}$. Each component is Zariski closed.

Proof. - Since $B$ has signature $(z, z)$, there exist $z$-dimensional real subspaces $V_{+}$and $V_{-}$of $\mathfrak{z}_{S}$ such that $B$ is respectively positive and negative definite on $V_{+}$and $V_{-}, B\left(V_{+}, V_{-}\right)=0$, and $\mathfrak{z}_{S}=V_{+}+V_{-}$is a direct sum. In fact, we take $V_{+}=\mathfrak{z} S \cap((1-i) \mathfrak{t})$ and $V_{-}=i V_{+}$. Denote by $\mathcal{N}$ the affine chart in $\operatorname{Gr}\left(z, \mathfrak{z}_{S}\right)$ around $V_{+}$given by

$$
\operatorname{End}\left(V_{+}, V_{+}\right) \longrightarrow \mathcal{N} \subset \operatorname{Gr}\left(z, \mathfrak{z}_{S}\right): \phi \longmapsto \mathfrak{l}_{\phi}=\left\{x+i \phi(x): x \in V_{+}\right\}
$$

Then it is easy to see that $\mathcal{L}_{\mathfrak{z} S} \subset \mathcal{N}$ and that $\mathfrak{l}_{\phi} \in \mathcal{L}_{\mathfrak{z} S}$ if and only if $\phi$ is an isometry of $\left(V_{+}, B\right)$. Thus we can identify $\mathcal{L}_{\mathfrak{z} S}$ with the subvariety $\mathrm{O}(z) \cong \mathrm{O}\left(V_{+}, B\right) \subset \mathcal{N}$. Under this identification, $\mathfrak{z}_{S} \cap \mathfrak{t}$ corresponds to the identity element in $\mathrm{O}(z)$, and thus $\mathcal{L}_{\mathfrak{z} S, 1}$ corresponds to the identity component of $\mathrm{O}(z)$. These results also follow from Corollary 14.13 in [26], or as the real case of Exercise B, pp. 102-103, in [2].

If two Lagrangian subspaces $V_{1}$ and $V_{2}$ of $\mathfrak{z}_{S}$ correspond to $\phi_{1}$ and $\phi_{2}$ in $\mathrm{O}\left(V_{+}, B\right)$ respectively, then $\operatorname{dim}\left(V_{1} \cap V_{2}\right)$ is equal to the multiplicity of 1 as an eigenvalue of $\phi_{1}^{-1} \phi_{2}$. Thus $V_{1}$ and $V_{2}$ lie in the same connected component of $\mathcal{L}_{\mathfrak{z}_{S}}$ if and only if $\operatorname{dim} V_{1} \cap V_{2}=z$ (mod 2). In particular, $\mathfrak{z}_{S} \cap \mathfrak{a}$, where $\mathfrak{a}=i \mathfrak{t}$, lies in $\mathcal{L}_{\mathfrak{z} S, 1}$ if and only if $z$ is even. This can also be seen from the fact that $\mathfrak{z}_{S} \cap \mathfrak{a}$ corresponds to minus the identity element in $\mathrm{O}(z)$.

\subsection{Real forms of $\mathfrak{g}$}

A real form of $\mathfrak{g}$ is clearly a Lagrangian subalgebra of $\mathfrak{g}$. Denote by $\mathcal{R}$ the set of all real forms of $\mathfrak{g}$. We will recall some facts about $\mathcal{R}$ in this section (see [22] or [1] for more details).

Let $\mathrm{Aut}_{\mathfrak{g}}$ be the group of complex linear automorphisms of $\mathfrak{g}$. Its identity component is the adjoint group $G=\operatorname{Int}_{\mathfrak{g}}$ of interior automorphisms of $\mathfrak{g}$. Let $\mathrm{Aut}_{D(\mathfrak{g})}$ be the automorphism group of the Dynkin diagram of $\mathfrak{g}$. It is well known that there is a split short exact sequence

$$
0 \longrightarrow \operatorname{Int}_{\mathfrak{g}} \longrightarrow \operatorname{Aut}_{\mathfrak{g}} \stackrel{\phi}{\longrightarrow} \operatorname{Aut}_{D(\mathfrak{g})} \longrightarrow 0
$$

Let $\theta$ be the Cartan involution of $\mathfrak{g}$ defined by the compact real form $\mathfrak{k}$. We will identify a real form $\mathfrak{g}_{0}$ of $\mathfrak{g}$ with the complex conjugation $\tau$ on $\mathfrak{g}$ such that $\mathfrak{g}_{0}=\mathfrak{g}^{\tau}$. Define

$$
\psi: \mathcal{R} \longrightarrow \operatorname{Aut}_{D(\mathfrak{g})}: \psi(\tau)=\phi(\tau \theta)=\phi(\theta \tau)
$$


To see that $\phi(\tau \theta)=\phi(\theta \tau)$, choose $g \in \operatorname{Int}_{\mathfrak{g}}$ such that $\tau_{1}=\operatorname{Ad}_{g} \tau \operatorname{Ad}_{g}^{-1}$ commutes with $\theta$ (see [10], Theorem III.7.1). Then we get

$$
\phi(\tau \theta)=\phi\left(\operatorname{Ad}_{g}^{-1} \tau_{1} \operatorname{Ad}_{g} \theta\right)=\phi\left(\tau_{1} \operatorname{Ad}_{g} \theta\right)=\phi\left(\tau_{1} \theta \theta^{-1} \operatorname{Ad}_{g} \theta\right)=\phi\left(\tau_{1} \theta\right)
$$

and similarly, $\phi(\theta \tau)=\phi\left(\theta \tau_{1}\right)$. Since $\tau_{1}$ commutes with $\theta$, we have $\phi(\tau \theta)=\phi(\theta \tau)$. In particular, we see that $\psi(\tau)$ is an involution.

Conversely, let $d$ be an involutive automorphism of the Dynkin diagram $D(\mathfrak{g})$. Then $d$ extends to a complex linear involution $\gamma_{d}$ of $\mathfrak{g}$ as follows: on $\mathfrak{h}$, let $\tilde{\gamma}_{d}$ be the automorphism induced via the Killing form by the complex linear extension of $d$. For $\alpha \in S\left(\Sigma^{+}\right)$, set $\tilde{\gamma}_{d}\left(E_{\alpha}\right)=E_{d \alpha}$. By [11], p. 75, there exists a unique complex Lie algebra automorphism $\gamma_{d}$ of $\mathfrak{g}$ extending $\tilde{\gamma}_{d}$. We claim that $\gamma_{d}$ commutes with $\theta$. Indeed, if $t_{\alpha}=\left[E_{\alpha}, E_{-\alpha}\right] \in \mathfrak{h}$, then since $t_{\alpha}$ corresponds to $\alpha$ using the Killing form, $\gamma_{d}\left(t_{\alpha}\right)=t_{d \alpha}$. Since $\gamma_{d}$ is a Lie algebra automorphism, it follows easily that $\gamma_{d}\left(E_{-\alpha}\right)=E_{-d \alpha}$. This implies $\gamma_{d}$ commutes with $\theta$ on generators $\left\{E_{\alpha}, E_{-\alpha} \mid \alpha \in S\left(\Sigma^{+}\right)\right\}$ of $\mathfrak{g}$, so $\gamma_{d}$ commutes with $\theta$. We denote the unique lift of $\gamma_{d}$ to $G$ by $\gamma_{d}$ also.

Set

$$
\mathcal{L}(\mathfrak{g}, d)=\psi^{-1}(d)
$$

Then

$$
\mathcal{R}=\bigcup_{d} \mathcal{L}(\mathfrak{g}, d)
$$

is a finite disjoint union, where $d$ runs over the set of all involutive diagram automorphisms of $\mathfrak{g}$.

Let $\tau_{d}=\gamma_{d} \theta=\theta \gamma_{d}$. Then $\tau_{d} \in \mathcal{L}(\mathfrak{g}, d)$. To describe all the elements in $\mathcal{L}(\mathfrak{g}, d)$, consider

$$
G^{-\tau_{d}}=\left\{g \in \operatorname{Int}_{\mathfrak{g}}:\left(\operatorname{Ad}_{g} \tau_{d}\right)^{2}=1\right\}=\left\{g \in G=\operatorname{Int}_{\mathfrak{g}}: \tau_{d}(g)=g^{-1}\right\} .
$$

Here $\tau_{d}$ also stands for the lifting of $\tau_{d}$ to $G$ by $\operatorname{Ad}_{\tau_{d}(g)}=\tau_{d} \operatorname{Ad}_{g} \tau_{d}$. If $g \in G^{-\tau_{d}}$, then $\operatorname{Ad}_{g} \tau_{d}$ is a real form of $\mathfrak{g}$ and $\psi\left(\operatorname{Ad}_{g} \tau_{d}\right)=d$, so $\operatorname{Ad}_{g} \tau_{d} \in \mathcal{L}(\mathfrak{g}, d)$. Conversely, if $\tau \in \mathcal{L}(\mathfrak{g}, d)$, then $\phi(\tau \theta)=\phi\left(\gamma_{d}\right)$, so $\tau=\operatorname{Ad}_{g} \tau_{d}$ for some $g \in \operatorname{Int}_{\mathfrak{g}}=\operatorname{ker}(\phi)$. But $\tau^{2}=1$, so $g \in G^{-\tau_{d}}$. Hence every real form $\tau$ in $\mathcal{L}(\mathfrak{g}, d)$ is of the form $\tau=\operatorname{Ad}_{g} \tau_{d}$ for some $g \in G^{-\tau_{d}}$.

LEMMA 3.10. - Every real form of $\mathfrak{g}$ is its own normalizer in $\mathfrak{g}$.

Proof. - The proof follows easily by considering the \pm 1 eigenspace decomposition $\mathfrak{g}=$ $\mathfrak{g}^{\tau} \oplus \mathfrak{g}^{-\tau}$ of $\tau$.

LEMMA 3.11. - $\mathcal{L}(\mathfrak{g}, d)$ is a smooth submanifold of $\operatorname{Gr}(n, \mathfrak{g})$ of dimension $\operatorname{dim}_{\mathbb{C}} \mathfrak{g}$.

Proof. - Note that $\operatorname{Int}_{\mathfrak{g}}$ acts on $\mathcal{L}(\mathfrak{g}, d)$ by the action $g \cdot \tau=\operatorname{Ad}_{g} \tau \operatorname{Ad}_{g}^{-1}$. The orbits of the larger group $\mathrm{Aut}_{\mathfrak{g}}$ on the set of all real forms are the equivalence classes of real forms, and there are only finitely many of them (see [22]). Since $\operatorname{Int}_{\mathfrak{g}}$ is the identity connected component of $\mathrm{Aut}_{\mathfrak{g}}$ and $\mathrm{Aut}_{\mathfrak{g}}$ has only finitely many components, it follows that $\mathrm{Int}_{\mathfrak{g}}$ has only finitely many orbits on the set of all real forms. Since $\mathcal{L}(\mathfrak{g}, d)$ is a subset of the set of all real forms, it follows that $\mathcal{L}(\mathfrak{g}, d)$ is a finite union of $\operatorname{Int}_{\mathfrak{g}}$ orbits. Now the action of $\operatorname{Int}_{\mathfrak{g}}$ on $\operatorname{Gr}(n, \mathfrak{g})$ by $(g, \mathfrak{l}) \mapsto g(\mathfrak{l})$ is smooth and $\mathcal{L}(\mathfrak{g}, d) \subset \operatorname{Gr}(n, \mathfrak{g})$ is a disjoint union of finitely many $\operatorname{Int}_{\mathfrak{g}}$-orbits. It follows that each $\operatorname{Int}_{\mathfrak{g}}$-orbit in $\mathcal{L}(\mathfrak{g}, d)$ is a smooth submanifold of $\operatorname{Gr}(n, \mathfrak{g})$. Moreover, by Lemma 3.10, all orbits have the same dimension. Thus, $\mathcal{L}(\mathfrak{g}, d)$ is a smooth submanifold of $\operatorname{Gr}(n, \mathfrak{g})$ of dimension $\operatorname{dim}_{\mathbb{C}} \mathfrak{g}$.

We will show later that the closure of $\mathcal{L}(\mathfrak{g}, d)$ in $\operatorname{Gr}(n, \mathfrak{g})$ (in the classical topology) is a smooth connected submanifold of $\operatorname{Gr}(n, \mathfrak{g})$. 
In Karolinsky's classification, the real forms $\mathfrak{m}_{S, 1}^{\tau}$ of $\mathfrak{m}_{S, 1}$ are such that the Killing form of $\mathfrak{g}$ restricted to $\mathfrak{m}_{S, 1}^{\tau}$ is real. We characterize such real forms in terms of their diagram automorphism.

Let $\mathfrak{c}=\mathfrak{h} \cap \mathfrak{m}_{S, 1}$. It is a Cartan subalgebra of $\mathfrak{m}_{S, 1}$. The Killing form of $\mathfrak{g}$ has nondegenerate restriction to $\mathfrak{c}$ and thus induces a bilinear form, denoted $B_{\mathfrak{c}}$ on $\mathfrak{c}^{*}$.

DEFINITION 3.12. - A diagram automorphism of the Dynkin diagram of $\mathfrak{m}_{S, 1}$ is said to be isometric if its complex linear extension to $\mathfrak{c}^{*}$ preserves $B_{\mathfrak{c}}$.

PROPOSITION 3.13. - The Killing form of $\mathfrak{g}$ is real on a real form $\mathfrak{m}_{S, 1}^{\tau}$ of $\mathfrak{m}_{S, 1}$ if and only if the diagram automorphism of the Dynkin diagram of $\mathfrak{m}_{S, 1}$ associated to $\mathfrak{m}_{S, 1}^{\tau}$ is isometric.

Proof. - The Cartan involution $\theta$ of $\mathfrak{g}$ defined by $\mathfrak{k}$ preserves $\mathfrak{m}_{S, 1}$ and restricts to give a Cartan involution of $\mathfrak{m}_{S, 1}$. Denote by $B$ the restriction of the Killing form of $\mathfrak{g}$ to $\mathfrak{m}_{S, 1}$. Let $\mathfrak{m}_{S, 1}^{\tau}$ be a real form of $\mathfrak{m}_{S, 1}$. Then $B$ is real on $\mathfrak{m}_{S, 1}^{\tau}$ if and only if $B(\tau x, \tau y)=\overline{B(x, y)}$ for all $x, y \in \mathfrak{m}_{S, 1}$, which is equivalent to $\tau \theta$ preserving $B$. By composing with an inner automorphism of $\mathfrak{m}_{S, 1}$ which necessarily preserves $B$, we can assume that $\tau \theta$ leaves $\mathfrak{c}$ invariant. Then it follows from the ad-invariance of $B$ that $\tau \theta$ preserves $B$ if and only if it preserves the restriction of $B$ to $\mathrm{c}$. By definition, this is equivalent to the automorphism of the Dynkin diagram of $\mathfrak{m}_{S, 1}$ induced by $\tau \theta$ being isometric.

Remark 3.14. - If $S$ contains exactly two orthogonal simple roots of $\mathfrak{g}$ with different lengths, then there is a diagram automorphism of $\mathfrak{m}_{S, 1}$ interchanging the $\mathfrak{s l}(2)$ 's corresponding to the simple roots which is not an isometry. An explicit description of all involutive isometries of the Dynkin diagram of $\mathfrak{m}_{S, 1}$ can be given in terms of involutive automorphisms of simple factors, and automorphisms which interchange simple factors $\mathfrak{r}_{1}$ and $\mathfrak{r}_{2}$ of $\mathfrak{m}_{S, 1}$ such that the restriction of the Killing form to $\mathfrak{r}_{1}$ coincides with the restriction of the Killing form to $\mathfrak{r}_{2}$ after composition with the automorphism. For example if $\mathfrak{m}_{S, 1} \cong \mathfrak{s o}(8) \oplus \mathfrak{s o}(8)$, then there are 22 such involutive automorphisms of the Dynkin diagram if the Killing form has the same restriction to each factor. Indeed, there are 4 involutions of the Dynkin diagram of $\mathfrak{s o}(8)$, so there are 16 involutions which preserve the two factors. If $\phi$ is an automorphism of the Dynkin diagram of $\mathfrak{s o}(8)$, then $(i, j) \mapsto\left(\phi^{-1}(j), \phi(i)\right)$ is an involution of the diagram of $\mathfrak{m}_{S, 1}$. Thus each of the 6 automorphisms of the Dynkin diagram of $\mathfrak{s o}(8)$ gives an involution of the Dynkin diagram of $\mathfrak{m}_{S, 1}$.

\subsection{Lagrangian data}

DEFinition 3.15. - A triple $(S, \varepsilon, d)$ is called Lagrangian datum if $S \subset S\left(\Sigma_{+}\right)$is a subset of the set of simple roots, $\varepsilon= \pm 1$, and $d$ is an involutive isometric diagram automorphism for the Dynkin diagram $D\left(\mathfrak{m}_{S, 1}\right)$ of $\mathfrak{m}_{S, 1}$. If $\mathfrak{l}=\operatorname{Ad}_{k}(\mathfrak{l}(S, V, \tau)), k \in K$, is a Lagrangian subalgebra, then $\mathfrak{l}$ has associated Lagrangian data $\Phi(\mathfrak{l})=(S, \varepsilon, d)$, where $\varepsilon=1$ if $V$ lies in the same connected component of $\mathcal{L}_{\mathfrak{z}_{S}}$ as $\mathfrak{z}_{S} \cap \mathfrak{t}$ and is -1 otherwise, and $d$ is the isometric diagram automorphism of $\mathfrak{m}_{S, 1}$ defined by $\tau$ (see Proposition 3.13). It follows from Proposition 3.3 that the triple $(S, \varepsilon, d)$ is determined by $\mathfrak{l}$.

Given Lagrangian datum $(S, \varepsilon, d)$, we let

$$
\mathcal{L}(S, \varepsilon, d)=\{\mathfrak{l}: \Phi(\mathfrak{l})=(S, \varepsilon, d)\} .
$$

Recall that $\mathcal{L}$ is the set of all Lagrangian subalgebras of $\mathfrak{g}$. Clearly, we have

$$
\mathcal{L}=\bigcup_{(S, \varepsilon, d)} \mathcal{L}(S, \varepsilon, d)
$$

$4^{\text {e }}$ SÉRIE - TOME $34-2001-\mathrm{N}^{\circ} 5$ 
Note that this is a finite disjoint union.

Proposition 3.16. - For each Lagrangian datum $(S, \varepsilon, d), \mathcal{L}(S, \varepsilon, d)$ is a smooth submanifold of the Grassmannian $\operatorname{Gr}(n, \mathfrak{g})$ of dimension $\operatorname{dim}(\mathfrak{k})+\frac{z(z-3)}{2}$, where $z=\operatorname{dim}_{\mathbb{C}} \mathfrak{z} S$, and it fibers over $G / P_{S}$ with the fiber being the product of $\mathcal{L}_{\mathfrak{z} S, \varepsilon}$ and $\mathcal{L}\left(\mathfrak{m}_{S, 1}, d\right)$.

Proof. - Let

$$
\mathcal{L}_{\mathfrak{p}_{S}}(S, \varepsilon, d)=\left\{\mathfrak{m}_{S, 1}^{\tau}+V+\mathfrak{n}_{S}: \mathfrak{m}_{S, 1}^{\tau} \in \mathcal{L}\left(\mathfrak{m}_{S, 1}, d\right), V \in \mathcal{L}_{\mathfrak{z} S, \varepsilon}\right\} \subset \operatorname{Gr}(n, \mathfrak{g}) .
$$

It can be identified with $\mathcal{L}\left(\mathfrak{m}_{S, 1}, d\right) \times \mathcal{L}_{\mathfrak{z} S, \varepsilon}$. Indeed, $\mathcal{L}\left(\mathfrak{m}_{S, 1}, d\right)$ is a submanifold of the Grassmannian $\operatorname{Gr}\left(m, \mathfrak{m}_{S, 1}\right)$, where $m=\operatorname{dim}_{\mathbb{C}}\left(\mathfrak{m}_{S, 1}\right), \mathcal{L}_{\mathfrak{z} S, \varepsilon}$ is a submanifold of $\operatorname{Gr}\left(z, \mathfrak{z}_{S}\right)$, and the direct sum map $\operatorname{Gr}\left(m, \mathfrak{m}_{S, 1}\right) \times \operatorname{Gr}\left(z, \mathfrak{z}_{S}\right) \rightarrow \operatorname{Gr}(n, \mathfrak{g}),(U, V) \mapsto U \oplus V \oplus \mathfrak{n}_{S}$ is a closed embedding.

We consider the multiplication map

$$
m: K \times_{K \cap P_{S}} \mathcal{L}_{\mathfrak{p}_{S}}(S, \varepsilon, d) \longrightarrow \operatorname{Gr}(n, \mathfrak{g}): m(k, \mathfrak{l})=\operatorname{Ad}_{k}(\mathfrak{l}) .
$$

The fiber product is a smooth manifold since it is a fiber bundle over $K / K \cap P_{S} \cong G / P_{S}$ with smooth fiber $\mathcal{L}_{\mathfrak{p}_{S}}(S, \varepsilon, d)$. The map $m$ is clearly smooth and proper, and the image of $m$ is $\mathcal{L}(S, \varepsilon, d)$ by the Karolinsky classification Theorem 3.2. We will show that $m$ is an immersion, and it will follow that $m$ is an embedding and that $\mathcal{L}(S, \varepsilon, d)$ is a smooth submanifold of $\operatorname{Gr}(n, \mathfrak{g})$.

The fact that $m$ is injective follows from Proposition 3.3. In order to show that the tangent map $m_{*}$ is injective, it suffices to show $m_{*}$ is injective at points of the form $(e, \mathfrak{l}(S, V, \tau))$ by $K$-equivariance. Recall that the tangent space at a plane $U$ to the $\operatorname{Grassmannian} \operatorname{Gr}(n, V)$ of $n$-planes in a space $V$ can be identified with $\operatorname{Hom}(U, V / U)$. Using this identification, the tangent space to the fiber product $K \times_{K \cap P_{S}} \operatorname{Gr}\left(n, \mathfrak{p}_{S}\right)$ at $\mathfrak{l}(S, V, \tau)$ is the quotient of $\mathfrak{k} \oplus \operatorname{Hom}\left(\mathfrak{l}(S, V, \tau), \mathfrak{p}_{S} / \mathfrak{l}(S, V, \tau)\right)$ by the relation $(X-Y, \xi(Y)+Z) \sim(X, Z)$, where $X \in \mathfrak{k}$, $Y \in \mathfrak{k} \cap \mathfrak{p}_{S}, \xi(Y)$ is the induced vector field at $\mathfrak{l}(S, V, \tau)$, and $Z \in \operatorname{Hom}\left(\mathfrak{l}(S, V, \tau), \mathfrak{p}_{S} / \mathfrak{l}(S, V, \tau)\right)$. Observe that for $Z$ to be tangent to the fiber $\mathcal{L}_{\mathfrak{p}_{S}}(S, \varepsilon, d)$, we must have $Z: \mathfrak{n}_{S} \rightarrow 0$. When we identify the tangent space to $\operatorname{Gr}(n, \mathfrak{g})$ at $\mathfrak{l}(S, V, \tau)$ with $\operatorname{Hom}(\mathfrak{l}(S, V, \tau), \mathfrak{g} / \mathfrak{l}(S, V, \tau))$, the tangent map is $m_{*}(X, Z)=\xi(X)+Z$, where $\xi(X)$ is the induced vector field. Now the claim that $m_{*}$ is injective follows since for any $X \notin \mathfrak{k} \cap \mathfrak{p}_{S}, \xi(X) \cdot \mathfrak{n}_{S} \not \subset \mathfrak{l}(S, V, \tau)$. To verify this last assertion, let $X \in \mathfrak{k} \backslash \mathfrak{k} \cap \mathfrak{p}_{S}$, and choose a maximal root $\alpha \notin[S]$ such that the projection $p_{-\alpha}(X)$ of $X$ to the $-\alpha$ root space $\mathfrak{g}_{-\alpha}$ is nonzero. Then $\left[X, \mathfrak{g}_{\alpha}\right]=\left[p_{-\alpha}(X), \mathfrak{g}_{\alpha}\right]+Y$ where $\langle\langle Y, Y\rangle\rangle=\left\langle\left\langle Y,\left[p_{-\alpha}(X), \mathfrak{g}_{\alpha}\right]\right\rangle\right\rangle=0$. Since $\left[p_{-\alpha}(X), \mathfrak{g}_{\alpha}\right]=\left[\mathfrak{g}_{\alpha}, \mathfrak{g}_{-\alpha}\right]$, which is a 2-dimensional real vector space on which the imaginary part of the Killing form is not isotropic, it follows that $\left[X, \mathfrak{g}_{\alpha}\right]$ is not isotropic. Thus, $\left[X, \mathfrak{g}_{\alpha}\right]$ is not contained in any Lagrangian subalgebra.

The dimension statement follows from Proposition 3.9 and Lemma 3.11.

Remark 3.17. - Note that $G$ preserves $\mathcal{L}(S, \varepsilon, d)$. The proof is similar to that of Corollary 3.7.

Example 3.18. - When $S$ is the set of all simple roots, we have $\mathfrak{m}_{S}=\mathfrak{g}$ and $\varepsilon$ can only be 1 , so $\mathcal{L}(S, \varepsilon, d)=\mathcal{L}(\mathfrak{g}, d)$.

Example 3.19. - For $\mathfrak{g}=\mathfrak{s l}(2, \mathbb{C})$, there are three $\mathcal{L}(S, \varepsilon, d)$ 's. First, $\mathcal{L}\left(S\left(\Sigma_{+}\right), 1\right.$, id $)$ is a disjoint union of the two symmetric spaces $\mathrm{SO}(3, \mathbb{C}) / \mathrm{SO}(3, \mathbb{R})$ and $\mathrm{SO}(3, \mathbb{C}) / \mathrm{SO}(2,1)$, where the first piece consists of compact real forms and the second piece consists of real forms isomorphic to $\mathfrak{s o}(2,1) . \mathcal{L}(\emptyset, 1, \mathrm{id})$ is the $\mathrm{SL}(2, \mathbb{C})$ orbit of $\mathfrak{t}+\mathfrak{n}$ and is isomorphic to $\mathbb{C} \mathrm{P}^{1}$. $\mathcal{L}(\emptyset,-1$, id $)$ is also isomorphic to $\mathbb{C} P^{1}$, and is the $\operatorname{SL}(2, \mathbb{C})$ orbit through $\mathfrak{a}+\mathfrak{n}$. As we will 
show in Section $6, \mathcal{L}(\emptyset, 1$, id $) \subset \overline{\mathcal{L}\left(S\left(\Sigma_{+}\right), 1, \text { id }\right)}$. This last closure can be identified with $\mathbb{R P}^{3}$, the projectivization of $2 \times 2$ Hermitian matrices.

In case $\mathfrak{g}=\mathfrak{s l}(3)$, there are eight $\mathcal{L}(S, \varepsilon, d)$ 's. $\mathcal{L}\left(S\left(\Sigma_{+}\right), 1\right.$,id $)$ is a union of components consisting of the real forms isomorphic to $\mathfrak{s u}(p, 3-p)$. It is a union of symmetric spaces. Let $\sigma$ be the nontrivial involution of the Dynkin diagram of $\mathfrak{s l}(3)$. Then $\mathcal{L}\left(S\left(\Sigma_{+}\right), 1, \sigma\right)$ consists of real forms isomorphic to $\mathfrak{s l}(3, \mathbb{R})$. There are four pieces of the form $\mathcal{L}\left(\alpha_{i}, \pm 1\right.$, id) corresponding to the two choices of $\alpha_{i}$ and the two choices of \pm 1 . Each of these pieces fibers over $G / P_{i}$ for a parabolic $P_{i}$ with the fiber being a symmetric space for $\operatorname{SL}(2, \mathbb{C})$. The final two pieces are of the form $\mathcal{L}(\emptyset, \pm 1$, id). These are bundles over the full flag variety $G / B$ with the fiber being a connected component of the orthogonal group $\mathrm{O}(2)$. The only nontrivial inclusions are $\mathcal{L}\left(\alpha_{i}, 1\right.$, id $) \subset \overline{\mathcal{L}\left(S\left(\Sigma_{+}\right), 1, \text { id }\right)}$.

Because of the fiber bundle decomposition of $\mathcal{L}(S, \varepsilon, d)$ and the fact that the base and $\mathcal{L}_{\mathfrak{z} S, \varepsilon}$ are compact, the study of the closure $\overline{\mathcal{L}(S, \varepsilon, d)}$ can be reduced to the study of $\overline{\mathcal{L}(\mathfrak{g}, d)}$ for $\mathfrak{g}$ semi-simple. In the following Sections 4 and 5, we show that $\overline{\mathcal{L}(\mathfrak{g}, d)}$ is a smooth connected submanifold of $\operatorname{Gr}(n, \mathfrak{g})$. We will also determine its decomposition into $G$-orbits. These results will be applied in Section 6 to show that $\overline{\mathcal{L}(S, \varepsilon, d)}$ is a smooth submanifold of $\operatorname{Gr}(n, \mathfrak{g})$.

\section{Extended signatures and the corresponding Lagrangian subalgebras of $\mathfrak{g}$}

\subsection{Extended $d$-signatures}

Let $d$ be an involutive diagram automorphism for the Dynkin diagram of $\mathfrak{g}$. In this section, we define Lagrangian subalgebras $\mathfrak{l}_{d, \sigma}$ associated to extended $d$-signatures $\sigma$ of the root system $\Sigma$, a notion that is a slight generalization of one from [23]. We will show that each $\mathfrak{l}_{d, \sigma}$ is in $\overline{\mathcal{L}(\mathfrak{g}, d)}$ and study the $G$-orbit through $\mathfrak{l}_{d, \sigma}$. In Section 5, we will show that every $\mathfrak{l}$ in $\overline{\mathcal{L}(\mathfrak{g}, d)}$ is $G$-conjugate to some $\mathfrak{l}_{d, \sigma}$.

Recall that $S\left(\Sigma_{+}\right)=\left\{\alpha_{1}, \alpha_{2}, \ldots, \alpha_{l}\right\}$ is the set of simple roots in $\Sigma_{+}$.

DEFINITION 4.1. - An extended d-signature of the root system $\Sigma$ is a map $\sigma: \Sigma \rightarrow\{-1,0,1\}$ satisfying $\sigma\left(d\left(\alpha_{i}\right)\right)=\sigma\left(\alpha_{i}\right)$ for $i=1,2, \ldots, l$, and

$$
\sigma(\alpha)=\prod_{i: m_{i} \neq 0} \sigma\left(\alpha_{i}\right)^{\left|m_{i}\right|}, \quad \text { where } \alpha=\sum_{i=1}^{l} m_{i} \alpha_{i} .
$$

We say that $\sigma$ is a $d$-signature if $\sigma(\alpha) \neq 0$ for any $\alpha \in \Sigma$.

An extended $d$-signature $\sigma$ is determined by its value on the simple roots. If $\sigma$ is an extended $d$-signature, let $\operatorname{supp}(\sigma)=\{\alpha \in \Sigma: \sigma(\alpha) \neq 0\}$. Then $S_{\sigma}:=S\left(\Sigma_{+}\right) \cap \operatorname{supp}(\sigma)$ is $d$-invariant. If we use $\left[S_{\sigma}\right]$ to denote the set of roots that are in the linear span of $S_{\sigma}$, then $\operatorname{supp}(\sigma)=\left[S_{\sigma}\right]$. Let

$$
S_{\sigma, 1}=\left\{\alpha_{i} \in S\left(\Sigma_{+}\right): \sigma\left(\alpha_{i}\right)=-1\right\}, \quad \check{\rho}_{1}=\sum_{\alpha_{i} \in S_{\sigma, 1}} \check{h}_{i} \in \mathfrak{a}
$$

where $\left\{\check{h}_{i}: i=1, \ldots, l\right\} \subset \mathfrak{a}$ is the set of fundamental coweights corresponding to the simple roots, namely $\alpha_{i}\left(\breve{h}_{j}\right)=\delta_{i, j}$ for $i, j=1, \ldots, l$. Then

$$
\sigma(\alpha)= \begin{cases}0, & \alpha \notin\left[S_{\sigma}\right], \\ (-1)^{\alpha\left(\check{\rho}_{1}\right)}, & \alpha \in\left[S_{\sigma}\right] .\end{cases}
$$


Conversely, for any $d$-invariant subset $S$ of $S\left(\Sigma_{+}\right)$and any $d$-invariant subset $S_{1}$ of $S$, there is an extended $d$-signature $\sigma$ such that $S=S_{\sigma}$ and $S_{1}=S_{\sigma, 1}$.

For an extended $d$-signature $\sigma$, let

$$
\mathfrak{m}_{\sigma}=\mathfrak{m}_{S_{\sigma}}=\mathfrak{h} \oplus\left(\bigoplus_{\alpha \in\left[S_{\sigma}\right]} \mathfrak{g}_{\alpha}\right), \quad \mathfrak{n}_{\sigma}=\mathfrak{n}_{S_{\sigma}}=\bigoplus_{\alpha \in \Sigma_{+}-\left[S_{\sigma}\right]} \mathfrak{g}_{\alpha}, \quad \mathfrak{p}_{\sigma}=\mathfrak{p}_{S_{\sigma}}=\mathfrak{m}_{\sigma} \oplus \mathfrak{n}_{\sigma}
$$

as in Section 3.1. Also let $\mathfrak{z}_{\sigma}=\mathfrak{z}_{S_{\sigma}}$ be the center of $\mathfrak{m}_{\sigma}$, and let $\mathfrak{m}_{\sigma, 1}=\left[\mathfrak{m}_{\sigma}, \mathfrak{m}_{\sigma}\right]$. Then $\sigma$ determines a complex linear involution $a_{\sigma}$ of $\mathfrak{m}_{\sigma}$ by

$$
\left.a_{\sigma}\right|_{\mathfrak{h}}=\mathrm{id},\left.\quad a_{\sigma}\right|_{\mathfrak{g}_{\alpha}}=\sigma(\alpha) \cdot \mathrm{id},
$$

where $\alpha \in \operatorname{supp}(\sigma)$. In other words,

$$
a_{\sigma}=\operatorname{Ad}_{\exp \left(\pi i \check{\rho}_{1}\right)} .
$$

Let $\tau_{d}=\gamma_{d} \theta$ be the conjugate linear involution of $\mathfrak{g}$ discussed in Section 3.4. Then it is routine to check that $\tau_{d, \sigma}:=a_{\sigma} \tau_{d}$ is a conjugate linear involution of $\mathfrak{m}_{\sigma}$ so the Lie algebra $\mathfrak{k}_{d, \sigma}=\mathfrak{m}_{\sigma}^{\tau_{d, \sigma}}$ is a real form of $\mathfrak{m}_{\sigma}$. Set

$$
\mathfrak{l}_{d, \sigma}=\mathfrak{k}_{d, \sigma}+\mathfrak{n}_{\sigma+} .
$$

It is easy to check that $\mathfrak{l}_{d, \sigma}$ is a Lagrangian subalgebra of $\mathfrak{g}$. Since $S_{\sigma}$ is $d$-invariant, $\mathfrak{m}_{\sigma}$ is invariant under $\gamma_{d}$. Regarded as an complex automorphism of $\mathfrak{m}_{\sigma, 1}, \gamma_{d}$ defines an automorphism of the Dynkin diagram of $\mathfrak{m}_{\sigma, 1}$ which is just $\left.d\right|_{S_{\sigma}}$. Let $\mathfrak{z}_{\sigma}^{\tau_{d}}$ be the fixed point set of $\tau_{d}$ restricted to $\mathfrak{z}_{\sigma}$. Set $\varepsilon=1$ if $\mathfrak{z}_{\sigma}^{\tau_{d}}$ lies in the same component as $\mathfrak{z}_{\sigma} \cap \mathfrak{t}$ and $\varepsilon=-1$ otherwise. Then, since $a_{\sigma}$ is an inner automorphism of $\mathfrak{m}_{\sigma, 1}$, we know that $\mathfrak{l}_{d, \sigma} \in \mathcal{L}\left(S_{\sigma}, \varepsilon,\left.d\right|_{S_{\sigma}}\right)$.

We can give a more explicit description of $\mathfrak{l}_{d, \sigma}$. Let $E_{\alpha}, E_{-\alpha}$, for $\alpha \in \Sigma_{+}$, be the root vectors chosen as in Section 3. Then

$$
\mathfrak{l}=\mathfrak{h}^{\tau_{d}}+\mathfrak{n}_{\sigma}+\operatorname{span}_{\mathbb{R}}\left\{E_{\alpha}-\sigma(\alpha) \gamma_{d}\left(E_{-\alpha}\right), i E_{\alpha}+i \sigma(\alpha) \gamma_{d}\left(E_{-\alpha}\right): \alpha \in \Sigma_{+} \cap\left[S_{\sigma}\right]\right\}
$$

Example 4.2. - When $\sigma(\alpha)=0$ for all $\alpha$, we have $\mathfrak{l}_{d, \sigma}=\mathfrak{h}^{\tau_{d}}+\mathfrak{n}$. On the other hand, $\sigma$ is a $d$-signature if and only if $\mathfrak{l}_{d, \sigma}$ is a real form of $\mathfrak{g}$. In this case, $\mathfrak{l}_{d, \sigma} \in \mathcal{L}(\mathfrak{g}, d)$.

\section{2. $d$-signatures and real forms in $\mathcal{L}(\mathfrak{g}, d)$}

We will now show that every real from in $\mathcal{L}(\mathfrak{g}, d)$ is $G$-conjugate to an $\mathfrak{l}_{d, \sigma}$ for some $d$-signature $\sigma$. The following proposition (and its proof) is analogous to a result of Karolinsky.

Proposition 4.3. - For any $\tau \in \mathcal{L}(\mathfrak{g}, d)$, there exist $g \in G$ and $t \in T^{\gamma_{d}}$ of order 2 such that

$$
\tau=\operatorname{Ad}_{g}\left(\operatorname{Ad}_{t} \tau_{d}\right) \operatorname{Ad}_{g^{-1}} .
$$

Proof. - By [10], Theorem III.7.1, we can find $v \in G$ so that $\operatorname{Ad}_{v} \tau \operatorname{Ad}_{v^{-1}}$ commutes with $\theta$. By [27], Theorem 1, there exists a Cartan subalgebra $\mathfrak{h}_{1}$ of $\mathfrak{g}$ which is $\operatorname{Ad}_{v} \tau \operatorname{Ad}_{v^{-1}}$ and $\theta$ stable. $\mathfrak{h}_{1}^{\theta}$ is the Lie algebra of a maximal torus of $K$, so by conjugacy of maximal tori in $K$, there exists $k \in K$ such that $\operatorname{Ad}_{k} \mathfrak{h}_{1}^{\theta}=\mathfrak{t}$. It follows that $\operatorname{Ad}_{k} \operatorname{Ad}_{v} \tau \operatorname{Ad}_{v^{-1}} \operatorname{Ad}_{k^{-1}}$ preserves $\mathfrak{t}$ and its complexification $\mathfrak{h}$. By conjugating by an element in $N_{K}(\mathfrak{h})$, we can further assume that a $K$-conjugate, denoted $\tau_{1}$, of $\operatorname{Ad}_{k} \operatorname{Ad}_{v} \tau \operatorname{Ad}_{v^{-1}} \operatorname{Ad}_{k^{-1}}$ preserves $\mathfrak{h}$ and maps the positive roots 
to the negative roots. Moreover, $\tau_{1}$ commutes with $\theta$, so $\tau_{1} \theta$ is a complex linear involution of $\mathfrak{h}$ preserving the set of positive roots. Hence, $\tau_{1} \theta=\operatorname{Ad}_{h} \gamma_{d}$ for some $h \in H$ (see [1], Proposition 2.8). Then $\tau_{1}=\operatorname{Ad}_{h} \tau_{d}$.

Since $\tau_{1}$ commutes with $\theta$, it follows that $\theta(h)=h$, so $h \in T$. Write $h=\exp (x)$, with $x=x_{1}+x_{2}, x_{1} \in \mathfrak{t}^{\gamma_{d}}, x_{2} \in \mathfrak{t}^{-\gamma_{d}}$. Since $\operatorname{Ad}_{h} \tau_{d}$ is a real form, it follows that $h \tau_{d}(h)=1$. Hence, $\exp \left(x_{1}\right) \exp \left(x_{2}\right) \exp \left(x_{1}\right) \exp \left(-x_{2}\right)=1$, so if we set $t=\exp \left(x_{1}\right), t^{2}=1$. Let $g_{2}=\exp \left(\frac{x_{2}}{2}\right)$. Then $\tau_{1}=\operatorname{Ad}_{g_{2}} \operatorname{Ad}_{t} \tau_{d} \operatorname{Ad}_{g_{2}^{-1}}$. Since $\tau_{1}$ is $G$-conjugate to $\tau$, this completes the proof.

COROLLARY 4.4. - Every real form $\tau \in \mathcal{L}(\mathfrak{g}, d)$ is conjugate to an $\mathfrak{l}_{d, \sigma}$ for some d-signature $\sigma$. Moreover,

$$
G \cdot \mathfrak{l}_{d, \sigma}=K A^{\gamma_{d}} \cdot \mathfrak{l}_{d, \sigma} .
$$

Proof. - By Proposition 4.3, there is $g \in G$ such that $\tau=\operatorname{Ad}_{g} \operatorname{Ad}_{t} \tau_{d} \operatorname{Ad}_{g^{-1}}$ with $t \in T^{\gamma_{d}}$ of order 2. The eigenvalues of $\operatorname{Ad}_{t}$ on each root space $\mathfrak{g}_{\alpha}$ are \pm 1 , and the map $\sigma: \Sigma \rightarrow\{1,-1\}$ defined this way is a $d$-signature because $\operatorname{Ad}_{\tau_{d}(t)}=\operatorname{Ad}_{t}$. By definition, the real form $\mathfrak{l}_{d, \sigma}$ is the fixed point set of the involution $\operatorname{Ad}_{t} \tau_{d}$. Therefore $\mathfrak{g}^{\tau}=\operatorname{Ad}_{g} \mathfrak{l}_{d, \sigma}$, which gives the first assertion. By Proposition 7.1.3 in [29], we can decompose $g$ as $g=k a g_{1}$, where $k \in K, a \in A^{\gamma_{d}}$ and $g_{1} \in G^{\mathrm{Ad}_{t} \tau_{d}}$. Thus

$$
\tau=\operatorname{Ad}_{k a}\left(\operatorname{Ad}_{t} \tau_{d}\right) \operatorname{Ad}_{a^{-1} k^{-1}}
$$

We now identify the $G$-orbits in $\mathcal{L}(\mathfrak{g}, d)$ with certain symmetric spaces.

Proposition 4.5. - Let $\tau$ be a real form of a semi-simple Lie algebra $\mathfrak{g}$ with its lifting to $G$ also denoted by $\tau$. Then the normalizer $N_{G}\left(\mathfrak{g}^{\tau}\right)$ of $\mathfrak{g}^{\tau}$ in $G$ coincides with $G^{\tau}:=\{g \in G$ : $\tau(g)=g\}$.

Proof. - Let $g \in G$. Then $g \in N_{G}\left(\mathfrak{g}^{\tau}\right)$ if and only if $\operatorname{Ad}_{g} \tau \operatorname{Ad}_{g^{-1}}=\tau$, or equivalently, $\tau \operatorname{Ad}_{g} \tau=\operatorname{Ad}_{g}$. But by the definition of the lifting of $\tau$ to $G, \tau \operatorname{Ad}_{g} \tau=\operatorname{Ad}_{\tau(g)}$. Thus $g \in N_{G}\left(\mathfrak{g}^{\tau}\right)$ if and only if $\operatorname{Ad}_{\tau(g)}=\operatorname{Ad}_{g}$. Since $G$ is the adjoint group, we see that $g \in N_{G}\left(\mathfrak{g}^{\tau}\right)$ if and only if $\tau(g)=g$ or $g \in G^{\tau}$.

COROLlary 4.6. - For a $d$-signature $\sigma$, the orbit $G \cdot \mathfrak{l}_{d, \sigma}$ is the semi-simple symmetric space $G / G^{\tau_{d, \sigma}}$.

Proof. - The above proposition implies that the stabilizer $N_{G}\left(\mathfrak{l}_{d, \sigma}\right)=G^{\tau_{d, \sigma}}$.

\subsection{Extended $d$-signatures and $\overline{\mathcal{L}(\mathfrak{g}, d)}$}

We have seen that the set $\mathcal{L}(\mathfrak{g}, d)$ is the union of the $G$-orbits $G \cdot \mathfrak{l}_{d, \sigma}$ as $\sigma$ runs over the set of all $d$-signatures. We now consider the $G$-orbits $G \cdot \mathfrak{l}_{d, \sigma}$, where $\sigma$ is any extended $d$-signature. We show that each such orbit is in $\overline{\mathcal{L}(\mathfrak{g}, d)}$, the closure of $\mathcal{L}(\mathfrak{g}, d)$ in the $\operatorname{Grassmannian} \operatorname{Gr}(n, \mathfrak{g})$ in the classical topology. In Section 5 we will show that $\overline{\mathcal{L}(\mathfrak{g}, d)}$ is precisely the union of all these $G$-orbits.

Lemma 4.7. - Suppose that $\sigma$ and $\sigma^{\prime}$ are two extended $d$-signatures such that $S_{\sigma} \subset S_{\sigma^{\prime}}$ and $\sigma(\alpha)=\sigma^{\prime}(\alpha)$ for all $\alpha \in S_{\sigma}$. Let $H \in \mathfrak{a}$ be such that $\alpha(H)=0$ for $\alpha \in S_{\sigma}$ and $\alpha(H)>0$ for all $\alpha \in S\left(\Sigma_{+}\right) \backslash S_{\sigma}$. Then

$$
\lim _{t \rightarrow+\infty} \operatorname{Ad}_{\exp (t H)} \mathfrak{l}_{d, \sigma^{\prime}}=\mathfrak{l}_{d, \sigma}
$$

in $\operatorname{Gr}(n, \mathfrak{g})$. Consequently, $G \cdot \mathfrak{l}_{d, \sigma} \subset \overline{G \cdot \mathfrak{l}_{d, \sigma^{\prime}}}$. 
Proof. - For $\alpha \in \Sigma_{+} \cap\left[S_{\sigma^{\prime}}\right]$, set $x_{\alpha}=E_{\alpha}-\sigma(\alpha) \gamma_{d}\left(E_{-\alpha}\right)$ and $y_{\alpha}=i E_{\alpha}+i \sigma(\alpha) \gamma_{d}\left(E_{-\alpha}\right)$. We know from Equation (14) that

$$
\mathfrak{l}_{d, \sigma^{\prime}}=\mathfrak{h}^{\tau_{d}}+\mathfrak{n}_{\sigma^{\prime}}+\operatorname{span}_{\mathbb{R}}\left\{x_{\alpha}, y_{\alpha}: \alpha \in \Sigma_{+} \cap\left[S_{\sigma^{\prime}}\right]\right\} .
$$

Pick $u_{0} \in \wedge^{p}\left(\mathfrak{h}^{\tau_{d}}+\mathfrak{n}_{\sigma^{\prime}}\right), u_{0} \neq 0$, where $p=\operatorname{dim}\left(\mathfrak{h}^{\tau_{d}}+\mathfrak{n}_{\sigma^{\prime}}\right)$. Let

$$
v_{0}=u_{0} \wedge \prod_{\alpha \in \Sigma_{+} \cap\left[S_{\sigma^{\prime}}\right]} x_{\alpha} \wedge y_{\alpha} \in \wedge^{n} \mathfrak{g}
$$

where $\mathfrak{g}$ is regarded as a $2 n$-dimensional real vector space. Then $\mathbb{R} v_{0} \in \mathbb{R} \mathrm{P}\left(\wedge^{n} \mathfrak{g}\right)$ is the image of $\mathfrak{l}_{d, \sigma^{\prime}}$ under the Plucker embedding of $\operatorname{Gr}(n, \mathfrak{g})$ into $\wedge^{n} \mathfrak{g}$. Since

$$
\begin{aligned}
& \operatorname{Ad}_{\exp (t H)} x_{\alpha}=\mathrm{e}^{t \alpha(H)}\left(E_{\alpha}-\sigma(\alpha) \mathrm{e}^{-2 t \alpha(H)} \gamma_{d}\left(E_{-\alpha}\right)\right), \\
& \operatorname{Ad}_{\exp (t H)} y_{\alpha}=\mathrm{e}^{t \alpha(H)}\left(i E_{\alpha}+i \sigma(\alpha) \mathrm{e}^{-2 t \alpha(H)} \gamma_{d}\left(E_{-\alpha}\right)\right),
\end{aligned}
$$

we see that $\lim _{t \rightarrow+\infty} \operatorname{Ad}_{\exp (t H)} v_{0}$ is the image of $\mathfrak{l}_{d, \sigma}$ in $\mathbb{R} \mathrm{P}\left(\wedge^{n} \mathfrak{g}\right)$ under the Plucker embedding of $\operatorname{Gr}(n, \mathfrak{g})$. Hence (15) holds.

COROLlaRY 4.8. - For every extended d-signature $\sigma$, we have

$$
G \cdot \mathfrak{l}_{d, \sigma} \subset \overline{\mathcal{L}(\mathfrak{g}, d)} \quad \text { and } \quad G \cdot\left(\mathfrak{h}^{\tau_{d}}+\mathfrak{n}\right) \cong G / B \subset \overline{G \cdot \mathfrak{l}_{d, \sigma}}
$$

where $B$ is the Borel subgroup of $G$ with Lie algebra $\mathfrak{h}+\mathfrak{n}$.

Proof. - Let $\sigma^{\prime}$ be any $d$-signature such that $\left.\sigma^{\prime}\right|_{S_{\sigma}}=\sigma$. Then by Lemma 4.7,

$$
G \cdot \mathfrak{l}_{d, \sigma} \subset \overline{G \cdot \mathfrak{l}_{d, \sigma^{\prime}}} \subset \overline{\mathcal{L}(\mathfrak{g}, d)} .
$$

On the other hand, let $\sigma_{0}$ be the extended $d$-signature such that $\sigma_{0}(\alpha)=0$ for all $\alpha$. Then $\mathfrak{l}_{d, \sigma_{0}}=\mathfrak{h}^{\tau_{d}}+\mathfrak{n}$. By Lemma 4.7, the $G$-orbit $G \cdot\left(\mathfrak{h}^{\tau_{d}}+\mathfrak{n}\right) \cong G / B$ is contained in $\overline{G \cdot \mathfrak{l}_{d, \sigma}}$.

Remark 4.9. - When $d$ is non-trivial and $\sigma$ is a $d$-signature, a continuous curve $\gamma(t)$ in $G \cdot \mathfrak{l}_{d, \sigma}$ with $\lim _{t \rightarrow+\infty} \gamma(t)=\mathfrak{h}^{\tau_{d}}+\mathfrak{n}$ can be used to show that the map $T: \mathcal{L} \rightarrow \mathcal{L}$ defined in Theorem 2.21 is not continuous. See also Remark 2.22.

We now give a more precise description of the $G$-orbit $G \cdot \mathfrak{l}_{d, \sigma}$ for any extended $d$-signature $\sigma$. Let $P_{\sigma}$ and $M_{\sigma, 1}$ be the connected subgroup of $G$ with Lie algebras $\mathfrak{p}_{\sigma}$ and $\mathfrak{m}_{\sigma, 1}$ respectively. Recall that $\tau_{d, \sigma}=a_{\sigma} \tau_{d}$ defines a real from of $\mathfrak{m}_{\sigma, 1}$ such that $\mathfrak{l}_{d, \sigma}=\mathfrak{m}_{\sigma, 1}^{\tau_{d, \sigma}}+\mathfrak{z}_{\sigma}^{\tau_{d}}+\mathfrak{n}_{\sigma}$.

Proposition 4.10. - For any extended d-signature $\sigma$, the $G$-orbit $G \cdot \mathfrak{l}_{d, \sigma}$ fibers over $G / P_{\sigma}$, and the fibers are diffeomorphic to the symmetric space $M_{\sigma, 1} / M_{\sigma, 1}^{\tau_{d, \sigma}}$. In particular, $\operatorname{dim}_{\mathbb{R}} G \cdot \mathfrak{l}_{d, \sigma}=\operatorname{dim}_{\mathbb{C}} \mathfrak{g}-\operatorname{dim}_{\mathbb{C} \mathfrak{z} \sigma}$. The G-orbit $G \cdot \mathfrak{l}_{d, \sigma}$ is compact if and only if $\sigma(\alpha)=0$ for all $\alpha \in \Sigma$.

Proof. - Let $\mathfrak{r}_{d, \sigma}$ and $R_{d, \sigma}$ be the normalizers of $\mathfrak{l}_{d, \sigma}$ in $\mathfrak{g}$ and $G$ respectively. Then $R_{d, \sigma} \subset P_{\sigma}$, and by Lemma 3.4, $\mathfrak{r}_{d, \sigma}=\mathfrak{m}_{\sigma, 1}^{\tau_{d, \sigma}}+\mathfrak{z} \sigma+\mathfrak{n}_{\sigma}$. It follows that the map

$$
G \cdot \mathfrak{l}_{d, \sigma} \longrightarrow G / P_{\sigma}: \operatorname{Ad}_{g} \mathfrak{l}_{d, \sigma} \longmapsto g P_{\sigma}
$$

is a well-defined fibration and its fibers are diffeomorphic to $P_{\sigma} / R_{d, \sigma} \cong M_{\sigma, 1} / M_{\sigma, 1}^{\tau_{d, \sigma}}$. The dimension for $G \cdot \mathfrak{l}_{d, \sigma}$ follows from the formula for $\mathfrak{r}_{d, \sigma}$. If $\sigma$ is not identically zero, the fiber 
of the projection from $G \cdot \mathfrak{l}_{d, \sigma}$ to $G / P_{\sigma}$ is not compact, so $G \cdot \mathfrak{l}_{d, \sigma}$ can not be compact. Thus $G \cdot \mathfrak{l}_{d, \sigma}$ is compact when and only when $\sigma(\alpha)=0$ for all $\alpha$.

Similarly to Corollary 4.4, we have

Proposition 4.11. - For any extended d-signature $\sigma, G \cdot \mathfrak{l}_{d, \sigma}=K A_{\sigma}^{\gamma_{d}} \cdot \mathfrak{l}_{d, \sigma}$, where

$$
A_{\sigma}^{\gamma_{d}}=\exp \left(\left(\mathfrak{a} \cap \mathfrak{m}_{\sigma, 1}\right)^{\gamma_{d}}\right) .
$$

Proof. - Let $M_{\sigma}$ and $N_{\sigma}$ be the connected subgroups of $G$ with Lie algebras $\mathfrak{m}_{\sigma}$ and $\mathfrak{n}_{\sigma}$ respectively. Then $P_{\sigma}=M_{\sigma} N_{\sigma}$. Since $K$ acts on $G / P_{\sigma}$ transitively, we can write any $g \in G$ as $g=k m n$, where $k \in K, m \in M_{\sigma}$ and $n \in N_{\sigma}$. Since $N_{\sigma}$ normalizes $\mathfrak{l}_{d, \sigma}$, we have $\operatorname{Ad}_{g} \mathfrak{l}_{d, \sigma}=\operatorname{Ad}_{k} \operatorname{Ad}_{m} \mathfrak{l}_{d, \sigma}$. Recall that $\mathfrak{l}_{d, \sigma}=\mathfrak{m}_{\sigma, 1}^{\tau_{d, \sigma}}+\mathfrak{z}_{\sigma}^{\tau_{d}}+\mathfrak{n}_{\sigma}$. Applying Corollary 4.4 to the real form $\mathfrak{m}_{\sigma, 1}^{\tau_{d, \sigma}}$ for $\mathfrak{m}_{\sigma, 1}$, we know that there exist $k_{1} \in K \cap M_{\sigma, 1}$ and $a \in A_{\sigma}^{\gamma_{d}}$ such that $\operatorname{Ad}_{m} \mathfrak{m}_{\sigma, 1}^{\tau_{d, \sigma}}=\operatorname{Ad}_{k_{1} a} \mathfrak{m}_{\sigma, 1}^{\tau_{d, \sigma}}$. Thus $\operatorname{Ad}_{m} \mathfrak{l}_{d, \sigma}=\operatorname{Ad}_{k_{1} a} \mathfrak{l}_{d, \sigma}$ and $\operatorname{Ad}_{g} \mathfrak{l}_{d, \sigma}=\operatorname{Ad}_{k k_{1} a} \mathfrak{l}_{d, \sigma}$.

\section{5. $\overline{\mathcal{L}(\mathfrak{g}, d)}$ as the real part of the De Concini-Procesi compactification $Z_{d}$ of $G$}

Let $d$ be an involutive diagram automorphism of the Dynkin diagram of $\mathfrak{g}$. In this section, we identify the variety $\overline{\mathcal{L}(\mathfrak{g}, d)}$ with the set of real points of a De Concini-Procesi compactification $Z_{d}$ of the group $G$. Since $Z_{d}$ is known to be smooth, it follows that $\overline{\mathcal{L}(\mathfrak{g}, d)}$ is a smooth irreducible algebraic subvariety of $\operatorname{Gr}(n, \mathfrak{g})$. We also show that $\overline{\mathcal{L}(\mathfrak{g}, d)}$ is connected and determine the $G$-orbits in $\overline{\mathcal{L}(\mathfrak{g}, d)}$.

\subsection{The complexification of $\mathfrak{g}$}

Regard $\mathfrak{g}$ as a real Lie algebra and denote its complex structure by $J_{0} \in \operatorname{End}_{\mathbb{R}}(\mathfrak{g})$. We may identify its complexification $\mathfrak{g}_{\mathbb{C}}$ with $\left(\mathfrak{g} \oplus \mathfrak{g}, J_{0} \oplus J_{0}\right)$ via the map

$$
\mathfrak{g}_{\mathbb{C}} \longrightarrow\left(\mathfrak{g} \oplus \mathfrak{g}, J_{0} \oplus J_{0}\right): x+i y \longmapsto\left(x+J_{0} y, \theta(x)+J_{0} \theta(y)\right), \quad x, y \in \mathfrak{g} .
$$

Under this identification, the complex conjugation operator $\tau$ on $\mathfrak{g}_{\mathbb{C}}$ becomes

$$
\tau(x, y)=(\theta(y), \theta(x)),
$$

with its set of real points realized as

$$
(\mathfrak{g} \oplus \mathfrak{g})^{\tau}=\{(x, \theta(x)): x \in \mathfrak{g}\} .
$$

If $\mathfrak{r} \subset \mathfrak{g}$ is a real subalgebra, then $\mathfrak{r}_{\mathbb{C}}=\mathfrak{r}+i \mathfrak{r}$ is regarded as a complex subalgebra of $\mathfrak{g} \oplus \mathfrak{g}$. For example, $\mathfrak{k}_{\mathbb{C}}$ is the diagonal subalgebra $\mathfrak{g}_{\Delta}=\{(x, x): x \in \mathfrak{g}\}$ and $(\mathfrak{t}+\mathfrak{n})_{\mathbb{C}}=\mathfrak{h}_{\Delta}+\mathfrak{n}_{1}+\mathfrak{n}_{-2}$, where $\mathfrak{n}_{-}=\theta(\mathfrak{n})$ and for a Lie subalgebra $\mathfrak{r}$ of $\mathfrak{g}$,

$$
\mathfrak{r}_{\Delta}=\{(x, x): x \in \mathfrak{r}\}, \quad \mathfrak{r}_{1}=\{(x, 0): x \in \mathfrak{r}\}, \quad \mathfrak{r}_{2}=\{(0, x): x \in \mathfrak{r}\} .
$$

The proof of the following lemma is straightforward.

Lemma 5.1. - For an extended d-signature $\sigma$, the complexification $\mathfrak{l}_{d, \sigma, \mathbb{C}}$ of $\mathfrak{l}_{d, \sigma}$ is

$$
\mathfrak{l}_{d, \sigma, \mathbb{C}}=\left\{\left(x, a_{\sigma} \gamma_{d}(x)\right): x \in \mathfrak{m}_{\sigma}\right\} \oplus \mathfrak{n}_{\sigma 1} \oplus \mathfrak{n}_{\sigma-2},
$$

where $\mathfrak{n}_{\sigma-}=\theta\left(\mathfrak{n}_{\sigma}\right)$. 
Recall that $\langle\langle\rangle$,$\rangle is the Killing form of \mathfrak{g}$. Consider the symmetric form $I$ on $\mathfrak{g} \oplus \mathfrak{g}$ given by

$$
I\left(\left(x_{1}, x_{2}\right),\left(y_{1}, y_{2}\right)\right)=\left\langle\left\langle x_{1}, y_{1}\right\rangle\right\rangle-\left\langle\left\langle x_{2}, y_{2}\right\rangle\right\rangle .
$$

Then $\mathfrak{l} \subset \mathfrak{g}$ is a real Lagrangian subalgebra of $\mathfrak{g}$ with respect to the imaginary part of the Killing form if and only if $\mathfrak{l}_{\mathbb{C}} \subset \mathfrak{g} \oplus \mathfrak{g}$ is a complex Lagrangian subalgebra with respect to $I$. If we denote by $\mathcal{L}_{\mathbb{C}}$ the set of all complex Lagrangian subalgebras of $\mathfrak{g} \oplus \mathfrak{g}$ with respect to $I$, then we have the injective map

$$
\mathcal{L} \longrightarrow \mathcal{L}_{\mathbb{C}}: \mathfrak{l} \longmapsto \mathfrak{l}_{\mathbb{C}}
$$

With respect to the adjoint action of $G$ on $\mathcal{L}$, we have

$$
\left(\operatorname{Ad}_{g} \mathfrak{l}\right)_{\mathbb{C}}=\operatorname{Ad}_{(g, \theta(g))}\left(\mathfrak{l}_{\mathbb{C}}\right)
$$

On the group level, we have the analogous identification $G_{\mathbb{C}} \cong G \times G$. We lift $\tau$ to an involution, also denoted by $\tau$, of $G \times G$. In this context, $G$ (as the set of real points) is identified with the fixed point set of $\tau$ as $\{(g, \theta(g)): g \in G\}$. Let $G_{\Delta, d}=\left\{\left(g, \gamma_{d}(g)\right): g \in G\right\}$. Then $(G \times G) / G_{\Delta, d}$ is an example of a complex symmetric space, and De Concini and Procesi [4] have exhibited a particular smooth compactification $Z_{d}$ of $(G \times G) / G_{\Delta, d}$.

\subsection{The De Concini-Procesi compactification $Z_{d}$}

Note that $G \times G$ acts on the Grassmannian of $n$-dimensional complex subspaces of $\mathfrak{g} \oplus \mathfrak{g}$ through the adjoint action, where $n=\operatorname{dim}_{\mathbb{C}} \mathfrak{g}$. Consider the $\gamma_{d}$-diagonal subalgebra

$$
\mathfrak{g}_{\Delta, d}=\left\{\left(x, \gamma_{d}(x)\right): x \in \mathfrak{g}\right\}
$$

of $\mathfrak{g} \oplus \mathfrak{g}$ and the orbit $(G \times G) \cdot \mathfrak{g}_{\Delta, d}$ inside the Grassmannian. The stabilizer subgroup of $G \times G$ at $\mathfrak{g}_{\Delta, d}$ is $G_{\Delta, d}$, so $(G \times G) \cdot \mathfrak{g}_{\Delta, d} \cong(G \times G) / G_{\Delta, d}$. By definition, the De Concini-Procesi variety is the closure (with respect to the Zariski or the classical topology) of $(G \times G) \cdot \mathfrak{g}_{\Delta, d}$ in the Grassmannian. It will be denoted by $Z_{d}$ and it is called the De Concini-Procesi compactification of $(G \times G) / G_{\Delta, d}$. It is a smooth complex manifold of complex dimension $n$ (see [4] for more details). Since the variety of complex Lagrangian subalgebras is $G \times G$ stable, it follows that every element in $Z_{d}$ is a complex Lagrangian subalgebra of $\mathfrak{g} \oplus \mathfrak{g}$ of dimension $n$.

It is known [4] that $G \times G$ has finitely many orbits in $Z_{d}$. We describe the orbits. Recall that $S\left(\Sigma_{+}\right)=\left\{\alpha_{1}, \ldots, \alpha_{l}\right\}$ is the set of all simple roots. Let $\eta: S\left(\Sigma_{+}\right) \rightarrow\{0,1\}$ be any map. Regarding $\eta$ as an extended signature for the trivial involution, we have the parabolic subalgebra $\mathfrak{p}_{\eta}=\mathfrak{m}_{\eta}+\mathfrak{n}_{\eta}$ of $\mathfrak{g}$. Set $\mathfrak{n}_{\eta_{-}}=\theta\left(\mathfrak{n}_{\eta}\right)$. Consider the subalgebra

$$
\mathfrak{g}_{d, \eta}=\left\{\left(x, \gamma_{d}(x)\right): x \in \mathfrak{m}_{\eta}\right\} \oplus \mathfrak{n}_{\eta_{1}} \oplus \gamma_{d} \mathfrak{n}_{\eta_{-2}} .
$$

It is easy to check that $\mathfrak{g}_{d, \eta} \in Z_{d}$. In particular, $\mathfrak{g}_{\Delta, d}=\mathfrak{g}_{d, \eta_{1}}$, where $\eta_{1}(\alpha)=1$ for all roots $\alpha$.

THEOREM 5.2 ([4]). - Every point $\mathfrak{r} \in Z_{d}$ is in a $G \times G$ orbit of $\mathfrak{g}_{d, \eta}$ for some $\eta$.

DEFINITION 5.3. - We say that a complex subalgebra of $\mathfrak{g} \oplus \mathfrak{g}$ has a real structure if it is the complexification of a real subalgebra of $\mathfrak{g}$ under the identification $\mathfrak{g}_{\mathbb{C}} \cong \mathfrak{g} \oplus \mathfrak{g}$. We use $Z_{d, \mathbb{R}}$ to denote the set of all Lie subalgebras in $Z_{d}$ that have a real structure.

Note that a complex subalgebra $\mathfrak{r}$ of $\mathfrak{g} \oplus \mathfrak{g}$ is in $Z_{d, \mathbb{R}}$ if and only if $\tau(\mathfrak{r})=\mathfrak{r}$, and in this case, $\mathfrak{r}=\left(\mathfrak{r}^{\tau}\right)_{\mathbb{C}}$, where $\mathfrak{r}^{\tau} \subset \mathfrak{g} \oplus \mathfrak{g}$, the fixed point set of $\tau$ in $\mathfrak{r}$, is identified with its image in $\mathfrak{g}$ 
under the projection $\mathfrak{g} \oplus \mathfrak{g} \rightarrow \mathfrak{g}:(x, y) \mapsto x$. We can regard $\tau$ as an anti-holomorphic involution on the Grassmannian $\operatorname{Gr}(n, \mathfrak{g} \oplus \mathfrak{g})$ of $n$-dimensional complex subspaces of $\mathfrak{g} \oplus \mathfrak{g}$. Then since $\tau\left(\mathfrak{g}_{\Delta, d}\right)=\mathfrak{g}_{\Delta, d}$ and since

$$
\tau \operatorname{Ad}_{\left(g_{1}, g_{2}\right)}=\operatorname{Ad}_{\left(\theta\left(g_{2}\right), \theta\left(g_{1}\right)\right)} \tau
$$

the subvariety $Z_{d}$ of $\operatorname{Gr}(n, \mathfrak{g} \oplus \mathfrak{g})$ is $\tau$-invariant, and $Z_{d, \mathbb{R}}$ is the set of fixed points of $\tau$. Therefore $Z_{d, \mathbb{R}}$ is a smooth and compact subvariety of $\operatorname{Gr}(n, \mathfrak{g} \oplus \mathfrak{g})$ (see Section 1.3.4 in [30]).

Note also that $\mathfrak{g}_{d, \eta} \in Z_{d, \mathbb{R}}$ if and only if $\eta$ is constant on the $d$-orbits, and in this case, $\mathfrak{g}_{d, \eta}=$ $\mathfrak{l}_{d, \eta, \mathbb{C}}$. On the other hand, for any extended $d$-signature $\sigma$, we have $\mathfrak{l}_{d, \sigma} \in(G \times G) \cdot \mathfrak{g}_{d,|\sigma|} \in Z_{d}$, so $\mathfrak{l}_{d, \sigma} \in Z_{d, \mathbb{R}}$.

\section{3. $G$-orbits in $Z_{d, \mathbb{R}}$}

Recall that for every Lagrangian subalgebra $\mathfrak{l} \subset \mathfrak{g}$,

$$
\left(\operatorname{Ad}_{g} \mathfrak{l}_{\mathbb{C}}=\operatorname{Ad}_{(g, \theta(g))}\left(\mathfrak{l}_{\mathbb{C}}\right), \quad \forall g \in G\right.
$$

Proposition 5.4. - Every $\mathfrak{r} \in Z_{d, \mathbb{R}}$ is in the G-orbit of an $\mathfrak{l}_{d, \sigma, \mathbb{C}}$ for some extended $d$-signature $\sigma$, where $G$ acts on $Z_{d, \mathbb{R}}$ by $g \cdot\left(\mathfrak{l}_{\mathbb{C}}\right)=\operatorname{Ad}_{(g, \theta(g))} \mathfrak{l}_{\mathbb{C}}$.

Proof. - Let $\mathfrak{r}=\operatorname{Ad}_{\left(g_{1}, g_{2}\right)} \mathfrak{g}_{d, \eta}$ for some $\eta$, so

$$
\mathfrak{r}=\left\{\left(\operatorname{Ad}_{g_{1}}\left(y+z_{1}\right), \operatorname{Ad}_{g_{2}} \gamma_{d}\left(y+z_{2}\right)\right): y \in \mathfrak{m}_{\eta}, z_{1} \in \mathfrak{n}_{\eta}, z_{2} \in \mathfrak{n}_{\eta_{-}}\right\}
$$

Since $\mathfrak{r}$ has a real structure, $\tau(\mathfrak{r})=\mathfrak{r}$, so $\left(\operatorname{Ad}_{\theta\left(g_{2}\right)} \tau_{d}\left(y+z_{2}\right), \operatorname{Ad}_{\theta\left(g_{1}\right)} \theta\left(y+z_{1}\right)\right)$ is in $\mathfrak{r}$, so that $\operatorname{Ad}_{\theta\left(g_{2}\right)} \tau_{d}\left(y+z_{2}\right)=\operatorname{Ad}_{g_{1}}\left(u+v_{1}\right)$ for some $u \in \mathfrak{m}_{\eta}$ and $v_{1} \in \mathfrak{n}_{\eta}$. But

$$
\mathfrak{p}_{\eta}=\left\{\theta\left(y+z_{2}\right): y \in \mathfrak{m}_{\eta}, z_{2} \in \mathfrak{n}_{\eta_{-}}\right\},
$$

so $\operatorname{Ad}_{g_{1}^{-1} \theta\left(g_{2}\right)} \gamma_{d}\left(\mathfrak{p}_{\eta}\right) \subset \mathfrak{p}_{\eta}$. Since $\gamma_{d}\left(\mathfrak{p}_{\eta}\right)$ is $G$-conjugate to $\mathfrak{p}_{\eta}$, it follows that $\gamma_{d}\left(\mathfrak{p}_{\eta}\right)=\mathfrak{p}_{\eta}$. Since $P_{\eta}$ is the normalizer of $\mathfrak{p}_{\eta}$, it follows that $g_{1}^{-1} \theta\left(g_{2}\right) \in P_{\eta}$, so $g_{2}=\theta\left(g_{1} p\right)$, for some $p \in P_{\eta}$. Thus,

$$
\mathfrak{r}=\left\{\left(\operatorname{Ad}_{g_{1}}\left(y+z_{1}\right), \operatorname{Ad}_{\theta\left(g_{1}\right) \theta(p)} \gamma_{d}\left(y+z_{2}\right)\right): y \in \mathfrak{m}_{\eta}, z_{1} \in \mathfrak{n}_{\eta}, z_{2} \in \mathfrak{n}_{\eta_{-}}\right\} .
$$

Thus, up to $G$-conjugacy,

$$
\mathfrak{r}=\left\{\left(\left(y+z_{1}\right), \operatorname{Ad}_{\theta(p)} \gamma_{d}\left(y+z_{2}\right)\right): y \in \mathfrak{m}_{\eta}, z_{1} \in \mathfrak{n}_{\eta}, z_{2} \in \mathfrak{n}_{\eta_{-}}\right\}
$$

and $\mathfrak{m}_{\eta}, \mathfrak{n}_{\eta}$ and $\mathfrak{n}_{\eta_{-}}$are $\gamma_{d^{-}}$stable. Write $\theta(p)=l u$ with $l \in M_{\eta}, u \in N_{\eta_{-}}$. Since

$$
\left\{\operatorname{Ad}_{u}\left(y+z_{2}\right): y \in \mathfrak{m}_{\eta}, z_{2} \in \mathfrak{n}_{\eta_{-}}\right\}=\left\{\left(y+w_{2}\right): y \in \mathfrak{m}_{\eta}, w_{2} \in \mathfrak{n}_{\eta_{-}}\right\}
$$

it follows that

$$
\mathfrak{r}=\left\{\left(\left(y+z_{1}\right), \operatorname{Ad}_{l} \gamma_{d}\left(y+z_{2}\right)\right): y \in \mathfrak{m}_{\eta}, z_{1} \in \mathfrak{n}_{\eta}, z_{2} \in \mathfrak{n}_{\eta_{-}}\right\}=\operatorname{Ad}_{(1, l)} \mathfrak{g}_{d, \eta} .
$$

We now use again the assumption that $\mathfrak{r}$ has a real structure and the facts that $\theta\left(\mathfrak{m}_{\eta}\right)=\mathfrak{m}_{\eta}$, $\theta\left(\mathfrak{n}_{\eta}\right)=\mathfrak{n}_{\eta_{-}}, M_{\eta}$ preserves the decompositions $\mathfrak{p}_{\eta}=\mathfrak{m}_{\eta}+\mathfrak{n}_{\eta}$ and $\theta\left(\mathfrak{p}_{\eta}\right)=\mathfrak{m}_{\eta}+\mathfrak{n}_{\eta_{-}}$. Since

$$
\tau\left(y+z_{1}, \operatorname{Ad}_{l} \gamma_{d}\left(y+z_{2}\right)\right)=\left(\operatorname{Ad}_{\theta(l)} \gamma_{d}\left(\theta(y)+\theta\left(z_{2}\right)\right), \theta(y)+\theta\left(z_{1}\right)\right),
$$


we see that

$$
\left.\left\{\operatorname{Ad}_{\theta(l)} \gamma_{d} y, y\right): y \in \mathfrak{m}_{\eta}\right\}=\left\{\left(y, \operatorname{Ad}_{l} \gamma_{d}(y)\right): y \in \mathfrak{m}_{\eta}\right\}=\left\{\left(\gamma_{d}\left(\operatorname{Ad}_{l^{-1}} y\right), y\right): y \in \mathfrak{m}_{\eta}\right\} .
$$

Hence, $\operatorname{Ad}_{\theta(l)} \gamma_{d}=\gamma_{d} \operatorname{Ad}_{l^{-1}}$, and it follows that $\left(\operatorname{Ad}_{l} \tau_{d}\right)^{2}=1$.

Now, by Proposition 4.3, after acting by an element of $M_{\eta}$, we may assume that $\mathfrak{r}=$ $\operatorname{Ad}_{(1, t)} \mathfrak{g}_{d, \eta}$, where $t \in T^{\gamma_{d}}$ is such that $t^{2}=1$. As before, let $\sigma_{t}(\alpha)$ be the eigenvalue of $\operatorname{Ad}_{t}$ on the root space $\mathfrak{g}_{\alpha}$. Then $\sigma_{t}$ is a $d$-signature and we can define a new extended $d$-signature $\sigma$ by $\sigma(\alpha)=\eta(\alpha) \sigma_{t}(\alpha)$. Then $\operatorname{Ad}_{(1, t)} \mathfrak{g}_{d, \eta}=\mathfrak{l}_{d, \sigma, \mathbb{C}}$ by Lemma 5.1 , which completes the proof of the proposition.

\subsection{The identification of $\overline{\mathcal{L}(\mathfrak{g}, d)}$ with $Z_{d, \mathbb{R}}$ and its geometry}

Let $d$ be any involutive diagram automorphism of the Dynkin diagram of $\mathfrak{g}$. Recall that $\overline{\mathcal{L}(\mathfrak{g}, d)}$ is the closure of $\mathcal{L}(\mathfrak{g}, d)$ in $\operatorname{Gr}(n, \mathfrak{g})$ in the classical topology. Denote by $\operatorname{Gr}(n, \mathfrak{g} \oplus \mathfrak{g})$ the Grassmannian of $n$-dimensional complex subspaces in $\mathfrak{g} \oplus \mathfrak{g}$.

THEOREM 5.5. - The map

$$
C: \operatorname{Gr}(n, \mathfrak{g}) \longrightarrow \operatorname{Gr}(n, \mathfrak{g} \oplus \mathfrak{g}): \mathfrak{l} \longmapsto \mathfrak{l}_{\mathbb{C}}
$$

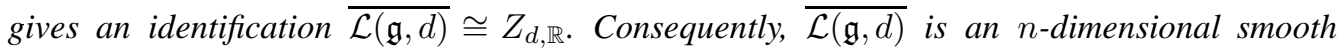
irreducible algebraic subvariety of $\operatorname{Gr}(n, \mathfrak{g})$.

Proof. - The map $C$ is $G$-equivariant, where $g \in G$ acts on $\operatorname{Gr}(n, \mathfrak{g})$ by $\operatorname{Ad}_{g}$ and on $\operatorname{Gr}(n, \mathfrak{g} \oplus \mathfrak{g})$ by $\operatorname{Ad}_{(g, \theta(g))}$. By Corollary 4.4 and the fact that every $\mathfrak{l}_{d, \sigma, \mathbb{C}} \in Z_{d, \mathbb{R}}$, we know that $C(\mathcal{L}(\mathfrak{g}, d)) \subset Z_{d, \mathbb{R}}$, so $C(\overline{\mathcal{L}(\mathfrak{g}, d)}) \subset Z_{d, \mathbb{R}}$ because $C$ is continuous. On the other hand, it follows from Proposition 5.4 and Corollary 4.8 that $Z_{d, \mathbb{R}} \subset C(\overline{\mathcal{L}(\mathfrak{g}, d)})$. Hence $C(\overline{\mathcal{L}(\mathfrak{g}, d)})=Z_{d, \mathbb{R}}$. Since $C$ is an embedding, and since $Z_{d, \mathbb{R}}$ is a smooth submanifold of $\operatorname{Gr}(n, \mathfrak{g} \oplus \mathfrak{g})$, we know that $\overline{\mathcal{L}(\mathfrak{g}, d)}$ is a smooth submanifold of $\operatorname{Gr}(n, \mathfrak{g})$. Since $\operatorname{Gr}(n, \mathfrak{g})$ is the set of real points of $\operatorname{Gr}(n, \mathfrak{g} \oplus \mathfrak{g})$ under $C$ and $Z_{d}$ is a complex algebraic subvariety of $\operatorname{Gr}(n, \mathfrak{g} \oplus \mathfrak{g})$, we see that $\overline{\mathcal{L}(\mathfrak{g}, d)}$ is a real algebraic subvariety of $\operatorname{Gr}(n, \mathfrak{g})$. It is irreducible because $Z_{d}$ is irreducible and $Z_{d}$ is the complexification of $\overline{\mathcal{L}(\mathfrak{g}, d)}$ (see [30], p. 9).

As a direct consequence of Theorem 5.5, we have:

COROLLARY 5.6. - Every Lagrangian subalgebra $\mathfrak{l}$ in $\overline{\mathcal{L}(\mathfrak{g}, d)}$ is $G$-conjugate to an $\mathfrak{l}_{d, \sigma}$ for some extended d-signature $\sigma$.

COROLlaRY 5.7. - The $G$-orbit $G \cdot\left(\mathfrak{h}^{\tau_{d}}+\mathfrak{n}\right) \cong G / B$ is the only closed orbit in $\overline{\mathcal{L}(\mathfrak{g}, d)}$, and it is contained in the closure of every $G$-orbit in $\overline{\mathcal{L}(\mathfrak{g}, d)}$.

Proof. - Clearly $G \cdot\left(\mathfrak{h}^{\tau_{d}}+\mathfrak{n}\right) \cong G / B$ is closed. By Corollary 5.6, every $G$-orbit $\mathcal{O}$ in $\overline{\mathcal{L}(\mathfrak{g}, d)}$ is of the form $G \cdot \mathfrak{l}_{d, \sigma}$ for some extended $d$-signature $\sigma$. It follows from Corollary 4.8 that $G \cdot\left(\mathfrak{h}^{\tau_{d}}+\mathfrak{n}\right) \subset \overline{\mathcal{O}}$. On the other hand, if $\mathcal{O}=G \cdot \mathfrak{l}_{d, \sigma}$ is closed, and therefore compact, then $\sigma(\alpha)=0$ for all $\alpha \in \Sigma$ by Proposition 4.10. Thus $\mathfrak{l}_{d, \sigma}=\mathfrak{h}^{\tau_{d}}+\mathfrak{n}$ and $\mathcal{O}=G \cdot\left(\mathfrak{h}^{\tau_{d}}+\mathfrak{n}\right)$.

Corollary 5.8. $-\overline{\mathcal{L}(\mathfrak{g}, d)}$ is connected.

Proof. - Since $G$ is connected, we know by Corollary 5.6 that every $\mathfrak{l} \in \overline{\mathcal{L}(\mathfrak{g}, d)}$ is pathconnected to some $\mathfrak{l}_{d, \sigma}$ which, by Lemma 4.7, is then path-connected to $\mathfrak{h}^{\tau_{d}}+\mathfrak{n}$.

COROLlary 5.9. - The Zariski closure of $\mathcal{L}(\mathfrak{g}, d)$ coincides with its closure in the classical topology. 
Proof. - We know from Theorem 5.5 that the closure $\overline{\mathcal{L}(\mathfrak{g}, d)}$ of $\mathcal{L}(\mathfrak{g}, d)$ in the classical topology is Zariski closed, so it contains the Zariski closure. But the Zariski closure contains the classical closure. It follows that they coincide.

Corollary 5.10. - For any real form $\tau \in \mathcal{L}(\mathfrak{g}, d)$, the Zariski closure of the G-orbit $G \cdot \mathfrak{g}^{\tau} \cong G / G^{\tau}$ in $\operatorname{Gr}(n, \mathfrak{g})$ is $\overline{\mathcal{L}(\mathfrak{g}, d)}$.

Proof. - Denote by $Z_{\tau}$ the Zariski closure of $G \cdot \mathfrak{g}^{\tau}$ in $\operatorname{Gr}(n, \mathfrak{g})$. Then $Z_{\tau} \subset \overline{\mathcal{L}(\mathfrak{g}, d)}$. Since $\overline{\mathcal{L}(\mathfrak{g}, d)}$ is irreducible and $Z_{\tau}$ has the same dimension as $\overline{\mathcal{L}(\mathfrak{g}, d)}$, it follows that $Z_{\tau}=\overline{\mathcal{L}(\mathfrak{g}, d)}$ (Exercise 1.10 in [9]).

Recall that a Lagrangian subalgebra $\mathfrak{l}$ of $\mathfrak{g}$ is a $\mathfrak{k}$-model point if $\mathfrak{k} \cap \mathfrak{l}=\mathfrak{k}_{\mathfrak{l}}$, where $\mathfrak{k}_{\mathfrak{l}}$ is the normalizer subalgebra of $\mathfrak{l}$ in $\mathfrak{k}$. Denote by $\mathbf{M}$ the set of all $\mathfrak{k}$-model points. We now show that $\mathbf{M}$ is the union of the $G$-orbits through some special subalgebras.

Denote by $\operatorname{Aut}_{D}^{I, S}$ the subset of all involutive, isometric automorphisms of the Dynkin diagram of the semi-simple subalgebra $\mathfrak{m}_{S, 1}$ of $\mathfrak{g}$. For each $d \in \mathrm{Aut}_{D}^{I, S}$, let $\mathcal{S}_{d, S}$ be the set of all $d$-signatures $\sigma$ of $\mathfrak{m}_{S, 1}$. If $d \in \operatorname{Aut}_{D}^{I, S}$, and $\sigma$ is a $d$-signature of $\mathfrak{m}_{S, 1}$, let $\tau_{d, \sigma}$ be the corresponding real form of $\mathfrak{m}_{S, 1}$. Let

$$
\mathfrak{l}_{d, \sigma}^{S}=\mathfrak{m}_{S, 1}^{\tau_{d, \sigma}}+\mathfrak{z} S \cap \mathfrak{t}+\mathfrak{n}_{S} .
$$

Note that $\mathfrak{l}_{d, \sigma}^{S} \in \mathcal{L}(S, 1, d)$.

PROPOSITION 5.11. - We have

$$
\mathbf{M}=\bigcup_{S \subset S\left(\Sigma_{+}\right)} \bigcup_{d \in \operatorname{Aut}_{D}^{I, S}} \bigcup_{\sigma \in \mathcal{S}_{d, S}} G \cdot \mathfrak{l}_{d, \sigma}^{S} .
$$

Proof. - Let $d \in$ Aut $_{D}^{I, S}$. It is easy to see from the definition of $\mathfrak{l}_{d, \sigma}^{S}$ and Proposition 3.5 that $\mathfrak{l}_{d, \sigma}^{S}$ is in $\mathbf{M}$ for every $\sigma \in \mathcal{S}_{d, S}$. Since $\mathbf{M}$ is $G$-invariant by Corollary 3.7, we know that $G \cdot \mathfrak{l}_{d, \sigma}^{S} \in \mathbf{M}$ for every such $\sigma$. Now assume that $\mathfrak{l} \in \mathbf{M}$. By Proposition 3.5, $\mathfrak{l}=\operatorname{Ad}_{k}\left(\mathfrak{m}_{S, 1}^{\tau}+\mathfrak{z} S \cap \mathfrak{t}+\mathfrak{n}_{S}\right)$ for some $k \in K, S \subset S\left(\Sigma_{+}\right)$and a real form $\tau$ of $\mathfrak{m}_{S, 1}$. By Corollary 4.4 applied to $\mathfrak{m}_{S, 1}$, $\mathfrak{m}_{S, 1}^{\tau}=\operatorname{Ad}_{m} \mathfrak{m}_{S, 1}^{\tau_{d, \sigma}}$ for some $d \in \operatorname{Aut}_{D}^{I, S}$, some $\sigma \in \mathcal{S}_{d, S}$, and some $m \in M_{S, 1}:=\left[M_{S}, M_{S}\right]$. Since $M_{S, 1}$ acts trivially on $\mathfrak{z} S$ and normalizes $\mathfrak{n}_{S}$, it follows that $\mathfrak{l}=\operatorname{Ad}_{k} \operatorname{Ad}_{m} \mathfrak{l}_{d, \sigma}^{S}$.

\subsection{Another description for $\overline{\mathcal{L}(\mathfrak{g}, \text { id })}$}

The set $\overline{\mathcal{L}(\mathfrak{g}, \text { id })}$ has been most important for applications. It will follow from the description of irreducible components in Section 6 that $\overline{\mathcal{L}(\mathfrak{g}, \text { id })}$ is the unique irreducible component of $\mathcal{L}$ containing $\mathfrak{k}$. In this section, we give another description of it.

Set

$$
\mathcal{L}_{0}=\{\mathfrak{l} \in \mathcal{L}: \operatorname{rank}(\mathfrak{k} \cap \mathfrak{l})=\operatorname{rank}(\mathfrak{k})\} .
$$

It is the set of Lagrangian subalgebras of $\mathcal{L}$ containing the Lie algebra of a maximal torus of $K$.

Proposition 5.12. $-\mathcal{L}_{0}=\overline{\mathcal{L}(\mathfrak{g}, \text { id })}$.

Proof. - Write $\mathfrak{l}_{\sigma}$ for $\mathfrak{l}_{d, \sigma}$ for $d$ trivial. First assume $\mathfrak{l}=\operatorname{Ad}_{g} \mathfrak{l}_{\sigma}$ lies in $\overline{\mathcal{L}(\mathfrak{g}, \text { id })}$. By Corollary 5.6 and Proposition 4.11, we can write $\mathfrak{l}=\operatorname{Ad}_{k} \operatorname{Ad}_{a} \mathfrak{l}_{\sigma}$, for $k \in K, a \in A$. But $\mathfrak{l}_{\sigma}$ contains $\mathfrak{t}$, so $\operatorname{Ad}_{k} \operatorname{Ad}_{a} \mathfrak{l}_{\sigma}$ contains $\operatorname{Ad}_{k}(\mathfrak{t})$, since $A$ acts trivially on $\mathfrak{t}$. Thus, $\mathfrak{l} \in \mathcal{L}_{0}$. 
Now, assume a Lagrangian subalgebra $\mathfrak{l}$ contains the Lie algebra of a maximal torus of $K$. By [12], we know $\mathfrak{l}=\operatorname{Ad}_{k}\left(\mathfrak{m}_{S, 1}^{\tau} \oplus V \oplus \mathfrak{n}_{S}\right)$, for some $(S, V, \tau)$. By the assumption on $\mathfrak{l}$, we may assume that $\mathfrak{m}_{S, 1}^{\tau} \oplus V$ contains $\mathfrak{t}$. Then $V=\mathfrak{t} \cap \mathfrak{z}_{S}$ and $\mathfrak{m}_{S, 1}^{\tau}$ contains a Cartan subalgebra of $\mathfrak{m}_{S, 1} \cap \mathfrak{k}$. But it is easy to show that if $\tau$ does not have trivial diagram automorphism, then $\mathfrak{m}_{S, 1}^{\tau}$ does not contain a Cartan subalgebra of $\mathfrak{m}_{S, 1} \cap \mathfrak{k}$. It follows easily that $\mathfrak{l} \in \overline{\mathcal{L}(\mathfrak{g}, \text { id })}$.

We remark that it follows that $G$ acts on $\mathcal{L}_{0}$, a fact that is not clear from the definition of $\mathcal{L}_{0}$.

Remark 5.13. - It is not difficult to show that every $\mathfrak{l}_{d, \sigma} \in \overline{\mathcal{L}(\mathfrak{g}, \mathrm{id})}$ is a $\mathfrak{k}$-model point. It follows from Corollary 3.7 and Corollary 5.6 that every $\mathfrak{l} \in \overline{\mathcal{L}(\mathfrak{g}, \text { id })}$ is a $\mathfrak{k}$-model point in $\mathcal{L}$. It follows from Section 2.3 that many familiar Poisson structures are contained in $\overline{\mathcal{L}(\mathfrak{g}, \text { id })}$ as $G$ or $K$ orbits with the Poisson structures being the restriction of the Poisson structure $\Pi$ on $\mathcal{L}$ defined in Section 2.2. For example, we can identify $G \cdot \mathfrak{k} \cong G / K$, and the Poisson structure induced by $\Pi$ on $G / K \cong A N$ is the negative of the Poisson structure $\pi_{A N}$ that makes $A N$ into the dual Poisson Lie group of $K$. More generally, by looking at $G$-orbits in $\mathcal{L}(\mathfrak{g}, d)$, we obtain in this manner a Poisson structure on $G / G_{0}$ for every real form $G_{0}$ of $G$. The Poisson manifolds arising from $K$-orbits in $\overline{\mathcal{L}(\mathfrak{g}, \text { id })}$ are studied in more detail in Section 7.

Remark 5.14. - Not all points in $\overline{\mathcal{L}(\mathfrak{g}, d)}$ are $\mathfrak{k}$-model points when $d$ is not trivial. The criterion for $\mathfrak{l}_{d, \sigma}$ to be a $\mathfrak{k}$-model point is that if $\sigma(\alpha)=0$, then $d(\alpha)=\alpha$.

In [7], we introduced certain $K$-invariant metrics $g_{\lambda}$ on $\mathrm{T}^{*}(K / T)$ for $\lambda \in \mathfrak{a}_{r}$, the set of elements in $\mathfrak{a}$ whose centralizer in $K$ is $T$. These metrics are important for showing that an operator $S$ introduced by Kostant is a limit of some Hodge Laplacians $S_{\lambda}$. The existence of this family simplifies the proof of Kostant's basic result that $\operatorname{Ker}(S)$ is isomorphic to $H^{*}(K / T)$. We remark that the metrics $g_{\lambda}$ can be understood in terms of the restriction of a Riemannian metric on the Riemannian symmetric space $G / K$. Since $\overline{\mathcal{L}(\mathfrak{g}, \text { id })}$ is a compactification of $G / K$ with closed orbit the flag manifold $G / B$, this observation provides evidence that embedding the Bruhat-Poisson structure on $G / B$ into the manifold $\overline{\mathcal{L}(\mathfrak{g}, \text { id })}$ is useful in Poisson geometry.

We give the construction of this metric. We can identify the tangent space of $G / K$ at $g K$ with $\operatorname{Ad}_{g}(i \mathfrak{k})$. The Killing form is positive definite at $\operatorname{Ad}_{g}(i \mathfrak{k})$, and we let $s$ be the metric on $G / K$ given by taking the square root of the Killing form metric on $\operatorname{Ad}_{g}(i \mathfrak{k})$.

Let $H_{\lambda} \in \mathfrak{a}$ be such that $\lambda(H)=\left(H_{\lambda}, H\right)$ and let $a_{\lambda}=\exp \left(H_{\lambda}\right)$. Then the $K$-orbit through $a_{\lambda} K \in G / K$ can be identified with $K / T$. If we restrict the above metric $s$ to a metric $s_{\lambda}$ on $K \cdot a_{\lambda} K \subset G / K$, and use $s_{\lambda}$ to identify the cotangent bundle with the tangent bundle, then one can show by easy calculations that we obtain the metric $g_{\lambda}$ from [7].

\section{Irreducible components of $\mathcal{L}$}

Recall from Section 3.5 that the variety $\mathcal{L}$ of all Lagrangian subalgebras of $\mathfrak{g}$ can be decomposed as the disjoint union

$$
\mathcal{L}=\bigcup_{(S, \varepsilon, d)} \mathcal{L}(S, \varepsilon, d)
$$

where, for the Lagrangian datum $(S, \varepsilon, d)$ (see Definition 3.15),

$$
\mathcal{L}(S, \varepsilon, d)=\left\{\operatorname{Ad}_{k}\left(\mathfrak{m}_{S, 1}^{\tau}+V+\mathfrak{n}_{S}\right): k \in K, \tau \in \mathcal{L}\left(\mathfrak{m}_{S, 1}, d\right), V \in \mathcal{L}_{\mathfrak{z}_{S, \varepsilon}}\right\}
$$

In this section, we will determine the irreducible components of $\mathcal{L}$ (as a real algebraic variety) using the closures $\overline{\mathcal{L}(S, \varepsilon, d)}$ of $\mathcal{L}(S, \varepsilon, d)$ in the Grassmannian $\operatorname{Gr}(n, \mathfrak{g})$ in the classical topology. 
We first combine results from Sections 3 and 5 to describe the geometry of the closures $\overline{\mathcal{L}(S, \varepsilon, d)}$.

THEOREM 6.1. - Each $\overline{\mathcal{L}(S, \varepsilon, d)}$ is a smooth connected submanifold of the Grassmannian $\operatorname{Gr}(n, \mathfrak{g})$ of dimension $\operatorname{dim}(\mathfrak{k})+\frac{z(z-3)}{2}$. It fibers over $G / P_{S}$, and the fiber is the product of $\mathcal{L}_{\mathfrak{z} S, \varepsilon}$ with $\overline{\mathcal{L}\left(\mathfrak{m}_{S, 1}, d\right)}$, the set of real points of a De Concini-Procesi variety.

Proof. - Recall from the proof of Proposition 3.16 that

$$
\mathcal{L}_{\mathfrak{p}_{S}}(S, \varepsilon, d):=\left\{\mathfrak{m}_{S, 1}^{\tau}+V+\mathfrak{n}_{S}: k \in K, \tau \in \mathcal{L}\left(\mathfrak{m}_{S, 1}, d\right), V \in \mathcal{L}_{\mathfrak{z} S, \varepsilon}\right\}
$$

It can be identified with the image of $\mathcal{L}\left(\mathfrak{m}_{S, 1}, d\right) \times \mathcal{L}_{\mathfrak{z}_{S, \varepsilon}} \subset \operatorname{Gr}\left(m, \mathfrak{m}_{S, 1}\right) \times \operatorname{Gr}\left(z, \mathfrak{z}_{S}\right)$ under the embedding

$$
E: \operatorname{Gr}\left(m, \mathfrak{m}_{S, 1}\right) \times \operatorname{Gr}\left(z, \mathfrak{z}_{S}\right) \longrightarrow \operatorname{Gr}(n, \mathfrak{g}):(U, V) \longmapsto U \oplus V \oplus \mathfrak{n}_{S}
$$

Thus $\overline{\mathcal{L}_{\mathfrak{p}_{S}}(S, \varepsilon, d)}$, the closure of $\mathcal{L}_{\mathfrak{p}_{S}}(S, \varepsilon, d)$ in $\operatorname{Gr}(n, \mathfrak{g})$ in the classical topology, can be identified with the image of $\overline{\mathcal{L}\left(\mathfrak{m}_{S, 1}, d\right)} \times \mathcal{L}_{\mathfrak{z} S, \varepsilon}$ under $E$. Thus $\overline{\mathcal{L}_{\mathfrak{p}_{S}}(S, \varepsilon, d)} \subset \operatorname{Gr}(n, \mathfrak{g})$ is smooth and connected by Theorem 5.5. Consider now the map

$$
m: K \times_{K \cap P_{S}} \overline{\mathcal{L}_{\mathfrak{p}_{S}}(S, \varepsilon, d)} \longrightarrow \operatorname{Gr}(n, \mathfrak{g}):(k, \mathfrak{l}) \longmapsto \operatorname{Ad}_{k} \mathfrak{l} .
$$

It is clear that $m$ is smooth and proper, and arguments similar to those used in the proof of Proposition 3.16 show that $m$ is an immersion. Thus $m$ is an embedding. But Karolinsky's Theorem 3.2 implies that the image of $m$ is $\overline{\mathcal{L}(S, \varepsilon, d)}$. Thus $\overline{\mathcal{L}(S, \varepsilon, d)}$ is a smooth connected submanifold of $\operatorname{Gr}(n, \mathfrak{g})$. The dimension statement is clear from Proposition 3.16.

PROPOSITION 6.2. $-\overline{\mathcal{L}(S, \varepsilon, d)} \subset \operatorname{Gr}(n, \mathfrak{g})$ is closed and irreducible in the Zariski topology.

Proof. - Since $\overline{\mathcal{L}_{\mathfrak{p}_{S}}(S, \varepsilon, d)}$ is Zariski closed in $\operatorname{Gr}(n, \mathfrak{g})$ by Proposition 3.9 and Theorem 5.5, it follows that $G \times{ }_{P_{S}} \overline{\mathcal{L}_{\mathfrak{p}_{S}}(S, \varepsilon, d)}$ is Zariski closed in $G \times{ }_{P_{S}} \operatorname{Gr}(n, \mathfrak{g})$. The map

$$
m: G \times{ }_{P_{S}} \operatorname{Gr}(n, \mathfrak{g}) \rightarrow \operatorname{Gr}(n, \mathfrak{g}): m(g, \mathfrak{l})=\operatorname{Ad}_{g} \mathfrak{l}
$$

is projective, so the image of $G \times{ }_{P_{S}} \overline{\mathcal{L}_{\mathfrak{p}_{S}}(S, \varepsilon, d)}$ under $m$ is Zariski closed, and irreducible since the domain is irreducible. Thus, the proposition follows from the proof of Theorem 6.1, since $G / P_{S} \cong K / K \cap P_{S}$.

Definition 6.3. - Lagrangian data $(S, \varepsilon, d)$ is said to be inessential if $S=S\left(\Sigma_{+}\right)-\left\{\alpha_{i}\right\}$, $d=\left.d^{\prime}\right|_{S}$ for some involutive diagram automorphism $d^{\prime}$ of $S\left(\Sigma_{+}\right)$, and $\varepsilon=1$. Otherwise, $(S, \varepsilon, d)$ is called essential.

Proposition 6.4. - Lagrangian data $(S, \varepsilon, d)$ is inessential if and only if

$$
\mathcal{L}(S, \varepsilon, d) \subset \partial \overline{\mathcal{L}\left(S^{\prime}, \varepsilon^{\prime}, d^{\prime}\right)}
$$

for some Lagrangian data $\left(S^{\prime}, \varepsilon^{\prime}, d^{\prime}\right)$.

Proof. - If $(S, \varepsilon, d)$ is inessential, then we claim $\mathcal{L}(S, \varepsilon, d) \subset \overline{\mathcal{L}\left(S\left(\Sigma_{+}\right), 1, d^{\prime}\right)}$, where $\left.d^{\prime}\right|_{S}=d$. Indeed, since $\operatorname{dim}_{\mathbb{C}}\left(\mathfrak{z}_{S}\right)=1$ and $\varepsilon=1$, the set $\mathcal{L}_{\mathfrak{z} S, \varepsilon}$ consists of only one point, namely $\mathfrak{z}_{S} \cap \mathfrak{t}$. It follows from Theorem 3.2 and Corollary 4.4 that each subalgebra in $\mathcal{L}(S, \varepsilon, d)$ is $G$-conjugate to 
$\mathfrak{m}_{S, 1}^{\tau_{d, \sigma}} \oplus \mathfrak{z} S \cap \mathfrak{t} \oplus \mathfrak{n}_{S}$ for some $d$-signature $\sigma$. But this algebra coincides with $\mathfrak{l}_{d^{\prime}, \sigma}$ which lies in $\partial \overline{\mathcal{L}\left(\mathfrak{g}, d^{\prime}\right)}$ by Lemma 4.7. Hence, $\mathcal{L}(S, \varepsilon, d)=\bigcup_{\sigma} G \cdot \mathfrak{l}_{d^{\prime}, \sigma} \subset \partial \overline{\mathcal{L}\left(\mathfrak{g}, d^{\prime}\right)}$.

Suppose that $\mathcal{L}(S, \varepsilon, d) \subset \partial \overline{\mathcal{L}\left(S^{\prime}, \varepsilon^{\prime}, d^{\prime}\right)}$. Then $S \subset S^{\prime}$ so $\operatorname{dim}_{\mathbb{C}}\left(\mathfrak{z}_{S}\right)>\operatorname{dim}_{\mathbb{C}}\left(\mathfrak{z}_{S^{\prime}}\right)$. Moreover, by Theorem 6.1 , we have

$$
\frac{\operatorname{dim}_{\mathbb{C}}\left(\mathfrak{z}_{S}\right)\left(\operatorname{dim}_{\mathbb{C}}\left(\mathfrak{z}_{S}\right)-3\right)}{2}<\frac{\operatorname{dim}_{\mathbb{C}}\left(\mathfrak{z}_{S^{\prime}}\right)\left(\operatorname{dim}_{\mathbb{C}}\left(\mathfrak{z}_{S^{\prime}}\right)-3\right)}{2} .
$$

It follows that $\operatorname{dim}_{\mathbb{C}}\left(\mathfrak{z}_{S}\right)=1$ or 2 and $\operatorname{dim}_{\mathbb{C}}\left(\mathfrak{z}_{S^{\prime}}\right)=0$. Thus, $\mathcal{L}\left(S^{\prime}, \varepsilon^{\prime}, d^{\prime}\right)=\mathcal{L}\left(\mathfrak{g}, d^{\prime}\right)$ consists of real forms. But every subalgebra in $\mathcal{L}(S, \varepsilon, d)$ is $G$ conjugate to some $\mathfrak{l}_{d, \sigma}$ by Corollary 5.6. Suppose first that $\operatorname{dim}_{\mathbb{C} \mathfrak{z} S}=1$. Since $\gamma_{d^{\prime}}$ acts by permutations on $\mathfrak{h}$, it follows that $\gamma_{d^{\prime}}$ acts trivially on $\mathfrak{z}_{S}$, so the Lagrangian subalgebra of $\mathfrak{z}_{S}$ associated by Karolinsky's classification with $\mathfrak{l}_{d, \sigma}$ is $\mathfrak{z}_{S} \cap \mathfrak{t}$. Thus, $\mathfrak{l}_{d, \sigma} \in \mathcal{L}\left(S, 1,\left.d^{\prime}\right|_{S}\right)$, and the assertion follows.

If $\operatorname{dim}_{\mathbb{C} \mathfrak{z} S}=2$, we will show that $\operatorname{dim}\left(\mathcal{L}(S, \varepsilon, d) \cap \overline{\mathcal{L}\left(\mathfrak{g}, d^{\prime}\right)}\right)<\operatorname{dim}(\mathcal{L}(S, \varepsilon, d))$, and it will follow that

$$
\mathcal{L}(S, \varepsilon, d) \not \subset \overline{\mathcal{L}\left(\mathfrak{g}, d^{\prime}\right)}=\overline{\mathcal{L}\left(S^{\prime}, \varepsilon^{\prime}, d^{\prime}\right)} .
$$

To prove the dimension claim, note

$$
\mathcal{L}(S, \varepsilon, d) \cap \overline{\mathcal{L}\left(\mathfrak{g}, d^{\prime}\right)} \subset \bigcup_{\sigma:\left[S_{\sigma}\right]=S} G \cdot \mathfrak{l}_{d, \sigma}
$$

so that $\operatorname{dim}\left(\mathcal{L}(S, \varepsilon, d) \cap Z_{d^{\prime}, \mathbb{R}}\right)=\operatorname{dim}_{\mathbb{C}} \mathfrak{g}-\operatorname{dim}_{\mathbb{C}} \mathfrak{z} S=\operatorname{dim}_{\mathbb{C}} \mathfrak{g}-2$ by Proposition 4.10. However, when $\operatorname{dim}_{\mathbb{C}}\left(\mathfrak{z}_{S}\right)=2, \operatorname{dim}(\mathcal{L}(S, \varepsilon, d))=\operatorname{dim}_{\mathbb{C}} \mathfrak{g}-1$ by Theorem 6.1 .

COROLlary 6.5.-

$$
\mathcal{L}=\bigcup_{\text {essential }(S, \varepsilon, d)} \overline{\mathcal{L}(S, \varepsilon, d)}
$$

is the decomposition of $\mathcal{L}$ into irreducible components.

Proof. - By Proposition 6.2, each $\overline{\mathcal{L}(S, \varepsilon, d)}$ is irreducible. Thus, the irreducible components are the $\overline{\mathcal{L}(S, \varepsilon, d)}$ 's not properly contained in any other $\overline{\mathcal{L}\left(S^{\prime}, \varepsilon^{\prime}, d^{\prime}\right)}$. By Proposition 6.4 , these correspond to essential data.

COROLlaRY 6.6. $-\overline{\mathcal{L}\left(S\left(\Sigma_{+}\right), 1, \mathrm{id}\right)} \cong \overline{\mathcal{L}(\mathfrak{g}, \mathrm{id})} \cong \mathcal{L}_{0}$ is the only irreducible component of $\mathcal{L}$ containing $\mathfrak{k}$.

Proof. - By Corollary 5.10, the Zariski closure of $G \cdot \mathfrak{k}$ is $\overline{\mathcal{L}(\mathfrak{g}, \text { id })}$, which is not contained in any other irreducible component by Corollary 6.5.

Note also that $\mathcal{L}$ itself is typically not smooth, because different irreducible components can intersect. This does not happen for $\mathfrak{s l}(2)$, but for $\mathfrak{s l}(3)$, the components $\overline{\mathcal{L}(\mathfrak{g}, \text { id })}$ and $\overline{\mathcal{L}(\emptyset, 1, \text { id })}$ intersect in the flag variety of $\mathrm{SL}(3, \mathbb{C})$.

\section{The Poisson structure $\Pi$ on $\mathcal{L}$}

In this section, we study some properties of the Poisson structure $\Pi$ on $\mathcal{L}$ defined in Section 2. More specifically, we relate $\Pi$ to the Bruhat Poisson structure and determine the $\left(K, \pi_{K}\right)$-homogeneous Poisson spaces defined by points in $\mathcal{L}_{0} \cong \overline{\mathcal{L}(\mathfrak{g}, \text { id })}$. 


\subsection{The fiber projection $\overline{\mathcal{L}(S, \varepsilon, d)} \rightarrow G / P_{S}$ is Poisson}

It is clear from the definition of $\Pi$ that every $G$-invariant smooth submanifold of $\mathcal{L}$ is a Poisson submanifold. Thus, each $\overline{\mathcal{L}(S, \varepsilon, d)}$ is a Poisson submanifold. On the other hand, equip $G / P_{S}$ with the Bruhat Poisson structure $\pi_{\infty}$, which is the unique $\left(K, \pi_{K}\right)$-homogeneous Poisson structure on $G / P_{S}$ that vanishes at the identity coset $e P_{S}$. Recall from Theorem 6.1 that we have the fiber bundle $\overline{\mathcal{L}(S, \varepsilon, d)} \rightarrow G / P_{S}$.

Proposition 7.1. - The fiber projection $\phi$ from $\overline{\mathcal{L}(S, \varepsilon, d)}$ to $G / P_{S}$ is a Poisson map.

Proof. - First, we observe that the projection $\phi$ is $G$-equivariant. Indeed, we can identify $K \times_{K \cap P_{S}} \overline{\mathcal{L}_{\mathfrak{p}_{S}}(S, \varepsilon, d)}$ with $G \times_{P_{S}} \overline{\mathcal{L}_{\mathfrak{p}_{S}}(S, \varepsilon, d)}$ via the obvious inclusion, and the map from $G \times{ }_{P_{S}} \overline{\mathcal{L}_{\mathfrak{p}_{S}}(S, \varepsilon, d)}$ to $\overline{\mathcal{L}(S, \varepsilon, d)}$ is given by the adjoint action $(g, \mathfrak{l}) \mapsto \operatorname{Ad}_{g} \mathfrak{l}$. Then the projection to $G / P_{S}$ is given by $(g, \mathfrak{l}) \mapsto g P_{S}$, which is obviously $G$-equivariant.

Recall that the Poisson structure on $\overline{\mathcal{L}(S, \varepsilon, d)}$ is induced by the element $\frac{1}{2} R \in \wedge^{2} \mathfrak{g}$ given in Section 2.2. Since $\phi$ is $G$-equivariant, it follows that $\phi_{*} \Pi$ is given by the bi-vector field on $G / P_{S}$ induced by $\frac{1}{2} R$, so we just have to check that $\frac{1}{2} R$ induces the Bruhat Poisson structure on $G / P_{S}$. It follows from the definition of the Drinfeld map that the Lagrangian subalgebra associated with the point $e P_{S}$ by $\pi_{\infty}$ is $\left(\mathfrak{k} \cap \mathfrak{p}_{S}\right) \oplus \mathfrak{n}_{S}$. By Theorem 2.23, the Drinfeld map

$$
\mathrm{P}:\left(G / P_{S}, \pi_{\infty}\right) \longrightarrow\left(K \cdot\left(\left(\mathfrak{k} \cap \mathfrak{p}_{S}\right) \oplus \mathfrak{n}_{S}\right), \Pi\right)
$$

is a Poisson map. The normalizer of $\left(\mathfrak{k} \cap \mathfrak{p}_{S}\right) \oplus \mathfrak{n}_{S}$ in $K$ is $K \cap P_{S}$, and it follows that the Drinfeld map is a diffeomorphism, so $\pi_{\infty}$ coincides with $\Pi$. Since the Poisson structure $\Pi$ is induced by $\frac{1}{2} R$, the result follows.

\section{2. $\left(K, \pi_{K}\right)$-homogeneous Poisson spaces determined by points in $\mathcal{L}_{0}$}

We now turn to the Poisson submanifold $\left(\mathcal{L}_{0}, \Pi\right)$, where $\mathcal{L}_{0} \cong \overline{\mathcal{L}(\mathfrak{g}, \text { id })}$ is the unique irreducible component of $\mathcal{L}$ that contains $\mathfrak{k}$. We study the $\left(K, \pi_{K}\right)$-homogeneous Poisson spaces determined by points in $\mathcal{L}_{0}$ (see Definition 2.9).

By Proposition 5.11 and Corollary 5.6, every point in $\mathcal{L}_{0}$ is a $\mathfrak{k}$-model point. It follows from the discussion in Section 2.3 that each $\mathfrak{l} \in \mathcal{L}_{0}$ can determine a number of $\left(K, \pi_{K}\right)$-homogeneous Poisson spaces, Indeed, let $N_{K}(\mathfrak{l})$ be the normalizer subgroup of $l$ in $K$. Then, for any subgroup $K_{1}$ of $N_{K}(\mathfrak{l})$ with the same Lie algebra $\mathfrak{l} \cap \mathfrak{k}$ as $N_{K}(\mathfrak{l})$, the space $K / K_{1}$ carries a unique Poisson structure $\pi$ such that the covering map

$$
P: K / K_{1} \longrightarrow K / N_{K}(\mathfrak{l}) \cong K \cdot \mathfrak{l} \subset \mathcal{L}_{0}: k K_{1} \longmapsto k N_{K}(\mathfrak{l})
$$

is a Poisson map. The space $\left(K / K_{1}, \pi\right)$ is automatically $\left(K, \pi_{K}\right)$-homogeneous, and the map $P$ is its Drinfeld map (see Definition 2.4). Examples of $K_{1}$ are $K_{1}=N_{K}(\mathfrak{l})$ or $K_{1}$ is the connected component of the identity of $N_{K}(\mathfrak{l})$. We can characterize these $\left(K, \pi_{K}\right)$-homogeneous Poisson spaces determined by points $\mathfrak{l} \in \mathcal{L}_{0}$ as follows.

Proposition 7.2. - All $\left(K, \pi_{K}\right)$-homogeneous Poisson spaces $\left(K / K_{1}, \pi\right)$ determined by points in $\mathcal{L}_{0}$ (see Definition 2.9) have the property that $K_{1}$ contains a maximal torus of $K$. Conversely, all $\left(K, \pi_{K}\right)$-homogeneous Poisson spaces with this property are determined by points in $\mathcal{L}_{0}$.

Proof. - The first part of the proposition follows from the definition of $\mathcal{L}_{0}$. Now, let $\left(K / K_{1}, \pi\right)$ be any $\left(K, \pi_{K}\right)$-homogeneous Poisson space such that $K_{1}$ contains a maximal torus of $K$. Then 
the Lie algebra $\mathfrak{k}_{1}$ of $K_{1}$ contains the Lie algebra of a maximal torus of $K$. Consider the Drinfeld map

$$
P: K / K_{1} \longrightarrow \mathcal{L} \text {. }
$$

Let $\mathfrak{l}=P\left(e K_{1}\right) \in \mathcal{L}$. Then by Drinfeld's Theorem 2.3, k by the definition of $\mathcal{L}_{0}$, and $\left(K / K_{1}, \pi\right)$ is determined by $\mathfrak{l}$.

The second part of Proposition 7.2 can be rephrased as the following.

Corollary 7.3. - Every $\left(K, \pi_{K}\right)$-homogeneous Poisson space $\left(K / K_{1}, \pi\right)$, where $K_{1}$ is a closed subgroup of $K$ containing a maximal torus of $K$, is a Poisson submanifold of $\left(\mathcal{L}_{0}, \Pi\right)$ up to a covering given by its Drinfeld map.

Remark 7.4. - Examples of $K_{1}$ in Proposition 7.3 are $K \cap Q$, where $Q$ is a parabolic subgroup of $G$, so the corresponding homogeneous space is a flag manifold $K /(K \cap Q) \cong G / Q$.

\subsection{The normalizer subgroup of $\mathfrak{l} \in \mathcal{L}_{0}$ in $K$}

We now study the normalizer subgroup $N_{K}(\mathfrak{l})$ of an arbitrary $\mathfrak{l} \in \mathcal{L}_{0}$ in $K$ and determine when it is connected. By Corollary 5.6 and Proposition 4.11, we can write $\mathfrak{l}=\operatorname{Ad}_{k} \operatorname{Ad}_{\exp H} \mathfrak{l}_{d, \sigma}$ for some $k \in K, H \in \mathfrak{a} \cap \mathfrak{m}_{\sigma, 1}$ and extended signature $\sigma$ for $d=\mathrm{id}$, the trivial diagram automorphism. In what follows, we will write $\mathfrak{l}_{\sigma}=\mathfrak{l}_{\mathrm{id}, \sigma}$ and call an extended signature for $d=\mathrm{id}$ simply an extended signature. We will write $\mathfrak{l}_{H, \sigma}=\operatorname{Ad}_{\exp H} \mathfrak{l}_{\sigma}$. Clearly, $\mathfrak{l}$ and $\mathfrak{l}_{H, \sigma}$ have isomorphic normalizers in $K$.

LemmA 7.5. - For $\mathfrak{l}_{H, \sigma}=\operatorname{Ad}_{\exp H} \mathfrak{l}_{\sigma}$, where $\sigma$ is an extended signature and $H \in \mathfrak{a} \cap \mathfrak{m}_{\sigma, 1}$,

$$
\mathfrak{l}_{H, \sigma} \cap \mathfrak{k}=\mathfrak{t}+\operatorname{span}_{\mathbb{R}}\left\{X_{\alpha}, Y_{\alpha}: \sigma(\alpha)=1, \alpha(H)=0\right\} .
$$

Proof. - This follows from the fact that

$$
\begin{aligned}
\operatorname{Ad}_{\exp H} \mathfrak{l}_{\sigma}=\mathfrak{t}+\mathfrak{n}_{\sigma} & +\operatorname{span}_{\mathbb{R}}\left\{\operatorname{Ad}_{\exp H} X_{\alpha}, \operatorname{Ad}_{\exp H} Y_{\alpha}: \sigma(\alpha)=1\right\} \\
+ & \operatorname{span}_{\mathbb{R}}\left\{i \operatorname{Ad}_{\exp H} X_{\alpha}, i \operatorname{Ad}_{\exp H} Y_{\alpha}: \sigma(\alpha)=-1\right\} .
\end{aligned}
$$

We now describe the normalizer subgroup of $\mathfrak{l}_{H, \sigma}$ in $K$.

Notation 7.6. - For an extended signature $\sigma$ and $H \in \mathfrak{a} \cap \mathfrak{m}_{\sigma, 1}$, let $\Sigma_{\sigma}=\{\alpha \in \Sigma: \sigma(\alpha)=1\}$. Let $W_{\sigma}$ be the subgroup of the Weyl group generated by the simple reflections corresponding to the simple roots in the support of $\sigma$. Let

$$
W_{H, \sigma}=\left\{w \in W_{\sigma}: w \Sigma_{\sigma}=\Sigma_{\sigma}, w H=H\right\} \subset W_{\sigma} \subset W .
$$

Let

$$
N^{\prime}\left(\mathfrak{l}_{H, \sigma}\right)=p^{-1}\left(W_{H, \sigma}\right),
$$

where $p: N_{K}(\mathfrak{t}) \rightarrow W=N_{K}(\mathfrak{t}) / T$ is the projection from the normalizer subgroup $N_{K}(\mathfrak{t})$ of $\mathfrak{t}$ in $K$ to the Weyl group. Finally, let $K_{H, \sigma}$ be the connected subgroup of $K$ with Lie algebra $\mathfrak{l}_{H, \sigma} \cap \mathfrak{k}$.

PROPOSITION 7.7. - For an extended signature $\sigma$ and $H \in \mathfrak{a} \cap \mathfrak{m}_{\sigma, 1}$, the normalizer subgroup $N_{K}\left(\mathfrak{l}_{H, \sigma}\right)$ of $\mathfrak{l}_{H, \sigma}=\mathrm{Ad}_{\exp H} \mathfrak{l}_{\sigma}$ is given by

$$
N_{K}\left(\mathfrak{l}_{H, \sigma}\right)=N^{\prime}\left(\mathfrak{l}_{H, \sigma}\right) K_{H, \sigma}=K_{H, \sigma} N^{\prime}\left(\mathfrak{l}_{H, \sigma}\right) .
$$


Proof. - It is clear from Lemma 7.5 that $N^{\prime}\left(\mathfrak{l}_{H, \sigma}\right)$ normalizes $\mathfrak{l}_{H, \sigma}$, so it normalizes $\mathfrak{l}_{H, \sigma} \cap \mathfrak{k}$ and the corresponding connected group $K_{H, \sigma}$. This implies the second equality, and the inclusion $K_{H, \sigma} N^{\prime}\left(\mathfrak{l}_{H, \sigma}\right) \subset N_{K}\left(\mathfrak{l}_{H, \sigma}\right)$.

Conversely, suppose that $k \in K$ normalizes $\mathfrak{l}_{H, \sigma}$. Then it normalizes the group $K_{H, \sigma}$, so $\operatorname{Ad}_{k} T$ is a maximal torus of $K_{H, \sigma}$, where $T$ is the maximal torus of $K$ with Lie algebra $\mathfrak{t}$. Thus there exists $k_{1} \in K_{H, \sigma}$ such that $\operatorname{Ad}_{k_{1}^{-1}} \operatorname{Ad}_{k} T=T$, i.e., $k_{1}^{-1} k \in N_{K}(T)=N_{K}(\mathfrak{t})$. Write $n=k_{1}^{-1} k$, so that $k=k_{1} n$. It remains to show that $n \in N^{\prime}\left(\mathfrak{l}_{H, \sigma}\right)$.

Denote by $w_{n}$ the Weyl group element $n T \in W$. Since $n$ normalizes $\mathfrak{l}_{H, \sigma}$, it normalizes its nilradical $\mathfrak{n}_{\sigma}$. Thus $w_{n} \in W_{\sigma}$. Now for each $\alpha \in\left[S_{\sigma}\right]$, the support of $\sigma$, consider the space

$$
V_{\alpha}=\mathfrak{l}_{H, \sigma} \cap\left(\mathfrak{g}_{\alpha} \oplus \mathfrak{g}_{-\alpha}\right) .
$$

By the description of the basis of $\mathfrak{l}_{\sigma}$, we know that the Killing form of $\mathfrak{g}$ restricted to $V_{\alpha}$ is either negative definite or positive definite depending on whether $\sigma(\alpha)=1$ or $\sigma(\alpha)=-1$. Now, since $n$ normalizes $\mathfrak{l}_{H, \sigma}$, it permutes the spaces $V_{\alpha}$, for $\alpha \in\left[S_{\sigma}\right]$. But $n$ preserves the Killing form, so $\sigma(\alpha)=1$ implies $\sigma\left(w_{n} \alpha\right)=1$. In other words, $w_{n} \Sigma_{\sigma}=\Sigma_{\sigma}$. It also follows that $n$ normalizes $\mathfrak{l}_{\sigma}$. Therefore we have

$$
\operatorname{Ad}_{\exp \left(w_{n} H\right)} \mathfrak{l}_{\sigma}=\operatorname{Ad}_{\exp H} \mathfrak{l}_{\sigma} .
$$

An easy calculation shows that this implies $\alpha(H)=\alpha\left(w_{n} H\right)$ for all $\alpha \in\left[S_{\sigma}\right]$. Since $H \in$ $\mathfrak{a} \cap \mathfrak{m}_{\sigma, 1}$ and $w_{n} \in W_{\sigma}$, it follows that $H=w_{n} H$. Therefore $w_{n} \in W_{H, \sigma}$, or, equivalently, $n \in N^{\prime}\left(\mathfrak{l}_{H, \sigma}\right)$.

COROLlary 7.8. - Let the notation be as in Notation 7.6. Then

$$
N_{K}\left(\mathfrak{l}_{H, \sigma}\right) / K_{H, \sigma} \cong N^{\prime}\left(\mathfrak{l}_{H, \sigma}\right) / N^{\prime}\left(\mathfrak{l}_{H, \sigma}\right) \cap K_{H, \sigma} .
$$

Remark 7.9. - For an extended signature $\sigma$, the group

$$
W_{0, \sigma}=\left\{w \in W_{\sigma}: w \Sigma_{\sigma}=\Sigma_{\sigma}\right\}
$$

contains the subgroup $R_{\sigma}$ generated by reflections $\left\{s_{\alpha}\right\}$ for $\alpha \in \Sigma_{\sigma}$ as a normal subgroup. Indeed, this follows from the formula for $s_{\alpha}$ and Formula (13) for $\sigma$. Set $Z_{\sigma}=W_{0, \sigma} / R_{\sigma}$. Regard $\sigma$ as a signature for the root system $\left[S_{\sigma}\right]$. Then $\sigma$ defines a signature for each irreducible subsystem of $\left[S_{\sigma}\right]$, and we can calculate $Z_{\sigma}$ separately for each irreducible subsystem. The group $Z_{\sigma}$ is computed for each simple Lie algebra in [23], Table 3, p. 80, and explicit elements are given. For example, when $\mathfrak{g}=\mathfrak{s l}(n, \mathbb{C})$, then if $\mathfrak{l}_{\sigma} \not \mathfrak{s u}(n / 2, n / 2)$, then $Z_{\sigma}$ is trivial, and if $\mathfrak{l}_{\sigma} \cong \mathfrak{s u}(n / 2, n / 2)$ then $Z_{\sigma}$ is a group with two elements. $Z_{\sigma}$ has no more than two elements except in the case when $\mathfrak{g}=\mathfrak{s o}(4 n, \mathbb{C})$ and $\mathfrak{l}_{\sigma} \cong \mathfrak{s o}(2 n, 2 n)$, when $Z_{\sigma}$ is the Klein 4-group. In particular, the group $W_{0, \sigma}$ can be calculated explicitly in each case. It follows that we can compute the group $W_{H, \sigma}$ explicitly.

\section{4. $\left(K, \pi_{K}\right)$-homogeneous Poisson structures on $K / T$}

In this section, we determine all $\left(K, \pi_{K}\right)$-homogeneous Poisson structures on the full flag variety $K / T$, where $T$ is the maximal torus of $K$ with Lie algebra $t$.

By Proposition 7.2, we only need to identify those $\mathfrak{l} \in \mathcal{L}_{0}$ such that $\mathfrak{l} \cap \mathfrak{k}=\mathfrak{t}$. We can assume $\mathfrak{l}=\mathfrak{l}_{H, \sigma}=\operatorname{Ad}_{\exp H} \mathfrak{l}_{\sigma}$, where $\sigma$ is an extended signature and $H \in \mathfrak{a} \cap \mathfrak{m}_{\sigma, 1}$, because the Poisson structure on $K / T$ determined by any $\mathfrak{l}=\mathrm{Ad}_{\mathrm{k}} \mathfrak{l}_{H, \sigma}$ for some $k \in K$ (such that $\mathfrak{l} \cap \mathfrak{k}=\mathfrak{t}$ ) will be $K$-equivariantly isomorphic to the one determined by $\mathfrak{l}_{H, \sigma}$. 
Proposition 7.10. - Let $\sigma$ be an extended signature and let $H \in \mathfrak{a} \cap \mathfrak{m}_{\sigma, 1}$. Let $\mathfrak{l}_{H, \sigma}=\operatorname{Ad}_{\exp H} \mathfrak{l}_{\sigma}$. Then $\mathfrak{l}_{H, \sigma} \cap \mathfrak{k}=\mathfrak{t}$ if and only if $\alpha(H) \neq 0$ for all $\alpha \in \Sigma_{\sigma}$.

Proof. - This is a direct consequence of Lemma 7.5.

For every $\mathfrak{l}_{H, \sigma}$ such that $\mathfrak{l}_{H, \sigma} \cap \mathfrak{k}=\mathfrak{t}$, denote by $\pi_{H, \sigma}$ the associated $\left(K, \pi_{K}\right)$-homogeneous Poisson structure on $K / T$.

COROLLARY 7.11. - The collection $\left\{\pi_{H, \sigma}\right\}$, as $\sigma$ runs over all extended signatures and as $H$ takes all elements in $\mathfrak{a} \cap \mathfrak{m}_{\sigma, 1}$ such that $\alpha(H) \neq 0$ when $\sigma(\alpha)=1$, gives all $\left(K, \pi_{K}\right)$-homogeneous Poisson structure on $K / T$.

An explicit formula for $\pi_{H, \sigma}$ is given in [21] as

$$
\pi_{H, \sigma}=p_{*} \pi_{K}+\frac{1}{2}\left(\sum_{\alpha \in\left[S_{\sigma}\right] \cap \Sigma_{+}} \frac{1}{1-\sigma(\alpha) \mathrm{e}^{2 \alpha(H)}} X_{\alpha} \wedge Y_{\alpha}\right)^{0}
$$

where $p: K \rightarrow K / T$ is the natural projection, and the second term on the right hand side is the $K$-invariant bi-vector field on $K / T$ whose value at $e=e T$ is the expression given in the parenthesis. The fact that these are all the $\left(K, \pi_{K}\right)$-homogeneous Poisson structures on $K / T$ up to $K$-equivariant isomorphisms is also proved in [21] by a different method. Namely, we show in [21] that every such Poisson structure comes from a solution to the Classical dynamical Yang-Baxter equation [8]. In [21], we also study some geometrical properties of these Poisson structures such as their symplectic leaves, modular vector fields, and moment maps for the $T$-action.

Recall from Proposition 7.7 and Notation 7.6 that when $\mathfrak{l}_{H, \sigma} \cap \mathfrak{k}=\mathfrak{t}$, the normalizer subgroup $N_{K}\left(\mathfrak{l}_{H, \sigma}\right)$ of $\mathfrak{l}_{H, \sigma}$ in $K$ lies in the normalizer subgroup of $\mathfrak{t}$ in $K$, and we have

$$
N_{K}\left(\mathfrak{l}_{H, \sigma}\right) / T=W_{H, \sigma}=\left\{w \in W_{\sigma}: w \Sigma_{\sigma}=\Sigma_{\sigma}, w H=H\right\} .
$$

When $W_{H, \sigma}$ is trivial, the Poisson manifold $\left(K / T, \pi_{H, \sigma}\right)$ embeds into $\left(\mathcal{L}_{0}, \Pi\right)$ as a Poisson submanifold. When $W_{H, \sigma}$ is not trivial, it follows from Proposition 2.28 that action of $W_{H, \sigma}$ on $K / T$ from the right defined by

$$
(K / T) \times W_{H, \sigma} \longrightarrow K / T:(k T, w) \longmapsto k w T
$$

is by Poisson isomorphisms. Thus, the group $W_{H, \sigma}$ gives symmetries of the Poisson structure. As we mentioned in Remark 7.9, this group can be calculated case by case.

Remark 7.12. - If $H \in \mathfrak{a}$ is regular in the sense that it is not fixed by any Weyl group element, then $W_{H, \sigma}$ is trivial for any $\sigma$. On the other hand, Borel and de Siebenthal showed that every nontrivial signature $\sigma$ corresponding to the trivial diagram automorphism can be put in a form such that $\sigma\left(\alpha_{k}\right)=-1$ for exactly one simple root $\alpha_{k}$ [3] or [23], Appendix. In particular, the group $W_{0, \sigma}$ contains the Weyl group of a maximal Levi subgroup, so for $W_{H, \sigma}$ to be trivial, $H$ cannot be fixed by any element in a maximal Levi subgroup, so in particular, $H$ can lie in at most one wall.

Example 7.13. - We can compute the Poisson structure $\Pi$ on $\mathcal{L}_{0}$ explicitly for the case of $\mathfrak{g}=\mathfrak{s l}(2, \mathbb{C})$. In this case, it follows from [4] that $\mathcal{L}_{0}$ can be $G=\operatorname{PSL}(2, \mathbb{C})$-equivariantly identified with $\mathbb{R} \mathrm{P}^{3}$, regarded as the projectivization of the space $\mathcal{H}$ of $2 \times 2$ Hermitian matrices, where the action of $G$ on $\mathcal{H}$ is by

$$
g \circ X=g X \bar{g}^{t}, \quad g \in G, X \in \mathcal{H} .
$$


The $R$-matrix $R \in \mathfrak{g} \wedge \mathfrak{g}$ (see Section 2.2) is explicitly given by

$$
R=-\frac{1}{2}\left(i h \wedge h-X_{\alpha} \wedge i E_{\alpha}+Y_{\alpha} \wedge E_{\alpha}\right),
$$

where

$$
h=\frac{1}{2 \sqrt{2}}\left(\begin{array}{cc}
1 & 0 \\
0 & -1
\end{array}\right), \quad X_{\alpha}=\frac{1}{2}\left(\begin{array}{cc}
0 & 1 \\
-1 & 0
\end{array}\right), \quad Y_{\alpha}=\frac{1}{2}\left(\begin{array}{cc}
0 & i \\
i & 0
\end{array}\right),
$$

and $E_{\alpha}=\frac{1}{2}\left(X_{\alpha}-i Y_{\alpha}\right)$. Denote by $v: \mathfrak{g} \rightarrow \chi^{1}(\mathcal{H})$ the Lie algebra anti-homomorphism defined by the above action of $G$ on $\mathcal{H}$, where $\chi^{1}(\mathcal{H})$ is the space of vector fields on $\mathcal{H}$. Then $\Pi=\frac{1}{2} v(R)$ is a Poisson structure on $\mathcal{H}$. Write an element of $\mathcal{H}$ as

$$
X=\left(\begin{array}{cc}
x & u+i v \\
u-i v & y
\end{array}\right)
$$

with $x, y, u, v \in \mathbb{R}$. Then the Poisson brackets for $\Pi$ are given by

$$
\begin{aligned}
& \{x, y\}=0, \quad\{x, u\}=-\frac{1}{4} y v, \quad\{x, v\}=\frac{1}{4} y u, \\
& \{y, u\}=\frac{1}{4} y v, \quad\{y, v\}=-\frac{1}{4} y u, \quad\{u, v\}=\frac{1}{8} y(y-x) .
\end{aligned}
$$

Note that

$$
c_{1}=x+y \quad \text { and } \quad c_{2}=x y-u^{2}-v^{2}
$$

are two Casimir functions. Hence all SU(2)-orbits are Poisson submanifolds. Since this Poisson structure is quadratic, it gives rise to one on $\mathbb{R} \mathrm{P}^{3}$, which is the Poisson structure $\Pi$ on $\mathcal{L}_{0}$. It can be checked that by looking at the $\mathrm{SU}(2)$-orbits through the points in $\mathbb{R} \mathrm{P}^{3}$ corresponding to

$$
\left(\begin{array}{ll}
b & 0 \\
0 & 1
\end{array}\right), \quad b \in \mathbb{R}, b \neq 1,
$$

we get all the $\left(K, \pi_{K}\right)$-homogeneous Poisson structures $\pi_{H, \sigma}$ on $\mathrm{SU}(2) / \mathrm{S}^{1}$, up to $K$-equivariant isomorphisms, as discussed in Section 7.4. By identifying $\mathrm{SU}(2) / \mathrm{S}^{1}$ with $\mathrm{S}^{2}=\{(x, y, z) \in$ $\left.\mathbb{R}^{3}: x^{2}+y^{2}+z^{2}=1\right\}$, these Poisson structures are given by

$$
\{x, y\}=\frac{1}{4}(x+2 a-1) z, \quad\{y, z\}=\frac{1}{4}(x+2 a-1) x, \quad\{z, x\}=\frac{1}{4}(x+2 a-1) y,
$$

for $a \in \mathbb{R}$. Note that the antipodal map is a symmetry for the case when $a=\frac{1}{2}$. This corresponds to the fact that the stabilizer subgroup in $\mathrm{SU}(2)$ of the point in $\mathbb{R} \mathrm{P}^{3}$ corresponding to $\left(\begin{array}{cc}1 & 0 \\ 0 & -1\end{array}\right)$ has two connected components.

\section{Remarks}

We define a Lagrangian pair in $\mathfrak{g}$ to be a pair of Lagrangian subalgebras $\left(\mathfrak{l}, \mathfrak{l}^{\prime}\right)$ such that $\mathfrak{l} \cap \mathfrak{l}^{\prime}=0$. Clearly a Lagrangian pair in $\mathfrak{g}$ is the same as a Lie bialgebra whose double is $\mathfrak{g}$ together with $\operatorname{Im}\langle\langle$,$\rangle (or a scalar multiple of it) as the invariant scalar product. Thus associated$ to each Lagrangian pair $\left(\mathfrak{l}, \mathfrak{l}^{\prime}\right)$ there is a Poisson structure $\Pi_{\mathfrak{l}, \mathfrak{l}^{\prime}}$ on the variety $\mathcal{L}$. There are many 
examples of Lagrangian pairs in $\mathfrak{g}$ other than the example $(\mathfrak{k}, \mathfrak{a}+\mathfrak{n})$. We can take, for example, $\mathfrak{l}=\mathfrak{t}+\mathfrak{n}_{-}$and $\mathfrak{l}^{\prime}=\mathfrak{a}+\mathfrak{n}$. Lagrangian pairs in $\mathfrak{g}$ have been classified by Delorme in [5] up to conjugacy by elements in $G$. In particular, it can be shown that for every $\mathfrak{l} \in \mathcal{L}$ there is at least one $\mathfrak{l}^{\prime}$ such that $\mathfrak{l}$ and $\mathfrak{l}^{\prime}$ form a Lagrangian pair. It would be interesting to study the geometries of the Poisson structures on $\mathcal{L}(\mathfrak{d})$ defined by different Lagrangian pairs and the relations among them. Note that our discussions in Sections 3-6 about the geometry of $\mathcal{L}$ are independent of the choice of the Lagrangian pair.

In general, assume that $\mathfrak{d}$ is a $2 n$-dimensional Lie algebra over the field $\mathbf{k}=\mathbb{R}$ or $\mathbb{C}$, and assume that $\langle$,$\rangle is a non-degenerate ad-invariant symmetric scalar product on \mathfrak{d}$. When $\mathbf{k}=\mathbb{R}$, we assume that the signature of $\langle$,$\rangle is (n, n)$. Then we have the notion of Lagrangian subalgebras in $\mathfrak{d}$, and we can form the algebraic variety $\mathcal{L}(\mathfrak{d})$. One example of $(\mathfrak{d},\langle\rangle$,$) , which is the$ complexification of our main example in this paper, is $\mathfrak{d}=\mathfrak{g} \oplus \mathfrak{g}$ with $\left\langle\left(x_{1}, x_{2}\right),\left(y_{1}, y_{2}\right)\right\rangle=$ $\left\langle\left\langle x_{1}, y_{1}\right\rangle\right\rangle-\left\langle\left\langle x_{2}, y_{2}\right\rangle\right\rangle$ for any semi-simple complex Lie algebra $\mathfrak{g}$. In this case, one can understand the geometry of $\mathcal{L}(\mathfrak{d})$ by using results in [13] and similar techniques as in Sections 3-6. Some connections between Lagrangian subalgebras of $\mathfrak{g}$ and of $\mathfrak{g} \oplus \mathfrak{g}$ and solutions to the classical dynamical Yang-Baxter equation studied by Etingof-Varchenko [8] and Schiffmann ([28]) can be found in [21]. More examples of $(\mathfrak{d},\langle\rangle$,$) can be found in [5], [24], [25]. The geometry of$ $\mathcal{L}(\mathfrak{d})$ is different for each example, and so are the classification of Lagrangian pairs in $\mathfrak{d}$ and the geometries of the Poisson structures $\Pi_{\mathfrak{l}, \mathfrak{l}^{\prime}}$. We plan to study these problems in additional cases in a sequel to this paper.

\section{Acknowledgements}

We would like to thank Eugene Karolinsky, Hermann Flaschka, and Dave Witte for useful conversations, and the Banach Center for its hospitality when some of these results were found. We would also like to thank the referees and Yvette Kosmann-Schwarzbach for useful comments. In addition, the first author would like to thank Northwestern University and the University of Chicago and the second author the Hong Kong University of Science and Technology for their hospitality during the preparation of the paper.

\section{REFERENCES}

[1] AdAms J., Barbasch D., Vogan D., The Langlands Classification and Irreducible Characters for Real Reductive Groups, Birkhäuser, 1992.

[2] Arbarello E., Cornalba M., Griffiths P., Harris J., Geometry of Algebraic Curves, Vol. 1, Springer-Verlag, 1985.

[3] Borel A., De Siebenthal J., Les sous-groupes fermes de rang maximum des groupes de Lie clos, Comment. Math. Helv. 23 (1949) 200-221.

[4] De Concini C., Procesi C., Complete symmetric varieties, in: Invariant Theory (Montecatini, 1982), Lect. Notes in Math., Vol. 996, Springer, Berlin, New York, 1983, pp. 1-44.

[5] Delorme P., Classification des triples de Manin pour les algèbres de Lie réductives complexes, math.QA/0003123.

[6] Drinfeld V.G., On Poisson homogeneous spaces of Poisson-Lie groups, Theor. Math. Phys. 95 (2) (1993) 226-227.

[7] Evens S., LU J.-H., Poisson harmonic forms, Kostant harmonic forms, and the $\mathrm{S}^{1}$-equivariant cohomology of $K / T$, Adv. Math. 142 (1999) 171-220.

[8] Etingof P., VARChenko A., Geometry and classification of solutions of the classical dynamical Yang-Baxter equation, Comm. Math. Phys. 192 (1998) 177-220.

[9] HARTShorne R., Algebraic Geometry, Springer-Verlag, 1977. 
[10] Helgason S., Differential Geometry, Lie Groups, and Symmetric Spaces, Academic Press, 1978.

[11] Humphreys J., Introduction to Lie Algebras and Representation Theory, Springer-Verlag, 1972.

[12] Karolinsky E., The classification of Poisson homogeneous spaces of compact Poisson Lie groups, Mathematical Physics, Analysis, and Geometry 3 (3/4) (1996) 274-289 (in Russian).

[13] Karolinsky E., A classification of Poisson homogeneous spaces of complex reductive Poisson-Lie groups, Banach Center Publ., Vol. 51, Polish Academy of Sciences, Warsaw, 2000.

[14] Korogodski L., Soibelman Y., Algebras of Functions on Quantum Groups, Part I, Mathematical Surveys and Monographs, Vol. 56, American Mathematical Society, 1998.

[15] Kostant B., Lie algebra cohomology and generalized Schubert cells, Ann. of Math. 77 (1) (1963) 72-144.

[16] Kostant B., Kumar S., The nil Hecke ring and cohomology of $G / P$ for a Kac-Moody group $G$, Adv. Math. 62 (3) (1986) 187-237.

[17] Lu J.-H., Weinstein A., Poisson Lie groups, dressing transformations, and Bruhat decompositions, J. Differential Geom. 31 (1990) 501-526.

[18] LU J.-H., Multiplicative and affine Poisson structures on Lie groups, PhD thesis, University of California, Berkeley, 1990.

[19] Lu J.-H., Poisson homogeneous spaces and Lie algebroids associated to Poisson actions, Duke Math. J. 86 (2) (1997) 261-304.

[20] LU J.-H., Coordinates on Schubert cells, Kostant's harmonic forms, and the Bruhat Poisson structure on $G / B$, Trans. Groups 4 (4) (1999) 355-374.

[21] LU J.-H., Classical dynamical $r$-matrices and homogeneous Poisson structures on $G / H$ and on $K / T$, Comm. Math. Phys. 212 (2000) 337-370.

[22] Onishchik A.L., Vinberg E.B. (Eds.), Structure of Lie Groups and Lie Algebras, Lie Groups and Lie Algebras III, Encyclopaedia of Mathematical Sciences, Vol. 41, Springer-Verlag, Berlin, 1994.

[23] Oshima T., SEKIGUCHI J., Eigenspaces of invariant differential operators on an affine symmetric space, Invent. Math. 57 (1980) 1-81.

[24] PANOV A., Manin triples of real simple Lie algebras, part 1, available as math.QA/9904156.

[25] PANov A., Manin triples of real simple Lie algebras, part 2, available as math.QA/9905028.

[26] Porteous I., Clifford Algebras and the Classical Groups, Cambridge University Press, 1995.

[27] Rossman W., The structure of semi-simple symmetric spaces, Canadian Math. J. 31 (1979) 157-180.

[28] Schiffmann O., On classification of dynamical $r$-matrices, Math. Res. Lett. 5 (1998) 13-31.

[29] SchlichtKrull H., Hyperfunctions and Harmonic Analysis on Symmetric Spaces, Birkhäuser, 1984.

[30] Silhol R., Real Algebraic Surfaces, Lect. Notes in Math., Vol. 1392, Springer-Verlag, 1989.

\footnotetext{
Sam Evens

Department of Mathematics,

University of Notre Dame,

Notre Dame, IN 46656, USA and

Department of Mathematics,

University of Arizona,

Tucson, AZ 85721, USA

E-mail: evens.1@nd.edu

Jiang-Hua LU

Department of Mathematics, University of Arizona,

Tucson, AZ 85721, USA

E-mail: jhlu@math.arizona.edu
} 